\title{
Uso de MCMC na Abordagem Bayesiana de Modelos ARCH e GARCH
}

\author{
Valéria Aparecida Martins Ferreira
}

Orientador: Prof. Dr. Marinho Gomes de Andrade Filho

Dissertação apresentada ao Instituto de Ciências Matemáticas e de Computação - ICMC-USP, como parte dos requisitos para obtenção do título de Mestre em Ciências de Computação e Matemática Computacional.

\author{
USP - São Carlos \\ $\mathrm{Março/2001}$
}




\section{A Comissão Julgadora:}

Prof. Dr. Marinho Gomes de Andrade Filho

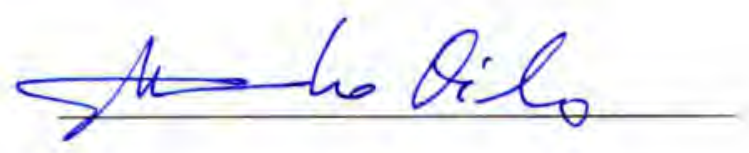

Prof. Dr. André Carlos Ponce de Leon F. de Carvalho

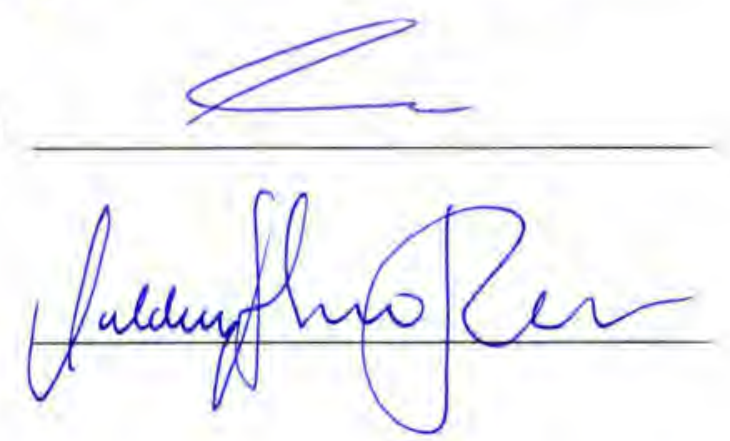

Prof. Dr. Valderio Anselmo Reisen 


\section{Agradecimentos}

A Deus, por todas as oportunidades e por estar sempre presente em todos os momentos de minha vida.

Ao meu orientador, Prof. Dr. Marinho Gomes de Andrade Filho, pela confiança, amizade, paciência e ensinamentos sempre demonstrados.

Aos meus pais, Adalberto e Maria, e à minha irmã Daniela, pelo apoio, incentivo e carinho demonstrados em todos os momentos.

Ao professor Dr. Luis Carlos Milan e ao professor Dr. André P. L. Carvalho pelas valiosas sugestões apresentadas em meu Exame de Qualificação.

À amiga Viviane, uma das responsáveis por esta 'virada'em minha vida, pelo apoio e amizade durante estes anos.

A amiga Cillene, pelo companheirismo, incentivo e apoio presente em todos os momentos.

Às amigas Alessandra, Andréa, Daniela, Eliane, Kátia e Vanessa que, mesmo à distância, sempre me incentivaram.

Às amigas Isabella e Ornella, pela ótima convivência durante estes anos.

Aos amigos Carlos, Emerson, Juan, Juana, Juliano, Manuel, Rúbia, Sandra e Ulisses, pelos ótimos momentos que compartilhamos.

À Adriana e à Sandra, da seção de convênios; às meninas da seção da pós: Laura, Marília e Beth; e a todos os funcionários do ICMC, por toda prestatividade e atenção dispensados.

À Fapesp e ao CNPq, pelo apoio financeiro. 


\section{Resumo}

Neste trabalho é descrito uma sequiência de procedimentos para estimar parâmetros e selecionar ordem de modelos Auto-Regressivos com heterocedasticidade, ARCH(p), e AutoRegressivos generalizados, $\operatorname{GARCH}(\mathrm{p}, \mathrm{q})$. As estimativas são obtidas utilizando duas técnicas: a inferência clássica e a bayesiana em conjunto com simulação de Monte Carlo em Cadeia de Markov (MCMC). Na análise bayesiana utilizamos densidades a priori normais para os parâmetros do modelo. Os métodos desenvolvidos foram aplicados em duas séries geradas e em três séries do mercado financeiro: Índice Bovespa, Telebrás e Cotação em Dólar Americano da moeda Iene Japonês. Em geral, as estimativas de máxima verossimilhança e bayesiana apresentaram resultados próximos. Porém, em algumas séries, o intervalo com 95\% de confiança para certos parâmetros do modelo apresentou valores negativos, o que viola as restrições impostas aos parâmetros dos modelos $\mathrm{ARCH}(\mathrm{p})$, destacando a vantagem da abordagem bayesiana. 


\begin{abstract}
In this work a sequence of procedures is described to estimate parameters, to select order and to forecast Autoregressive Conditional Heteroskedasticity $\operatorname{ARCH}(\mathrm{p})$ and generalized ARCH, GARCH(p,q), models. The estimates are obtained by using both classical inference techniques via maximum likelihood estimation and Bayesian inference approach jointly with simulation of Monte Carlo Markov Chain (MCMC). In the Bayesian analysis we use normal prior densities for the parameters of the model. The applications for the developed methods were made in a generated series and in three series of the Brazilian finance market: Index Bovespa, Telebrás and Quotation in American Dollar of the Japanese Yen. In general, the maximum likelihood and Bayesian estimates are similar. However, in some series, the $95 \%$ confidence intervals for some parameters of the model, presented negative values, violating the constraints imposed to the parameters of the ARCH(p) models, highlighting certain advantage of the Bayesian approach.
\end{abstract}




\section{Índice}

CAPÍTULO 1 : INTRODUÇÃO.....................................................................................................................1

CAPÍTULO 2: MODELOS ARCH E GARCH.............................................................................................

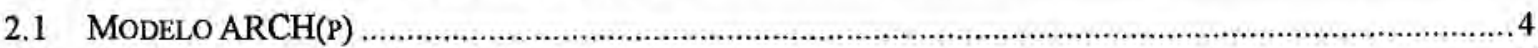

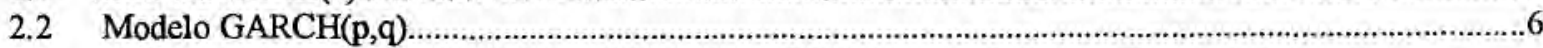

CAPÍTULO 3: ESTIMADORES DE MÁXIMA VEROSSIMILHANÇA DOS MODELOS ARCH(P) E

GARCH(P,Q)

3.1 ESTIMADORES DE MÁXIMA VEROSSIMILHANÇA DOS MODELOS ARCH(P) .........................................

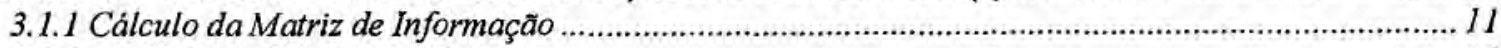

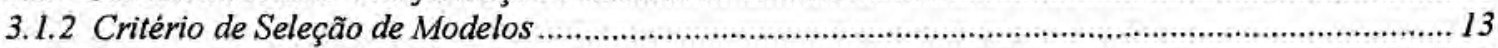

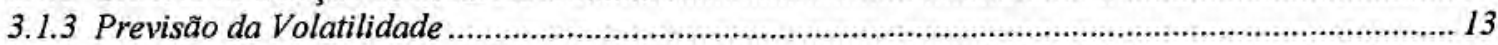

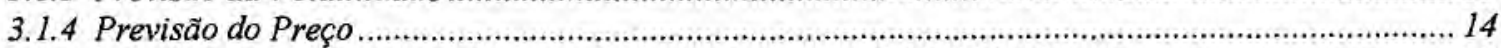

3.2 ESTIMADORES DE MÁXIMA VEROSSIMILHANÇA DOS MODELOS GARCH(P,Q) ....................................... 16

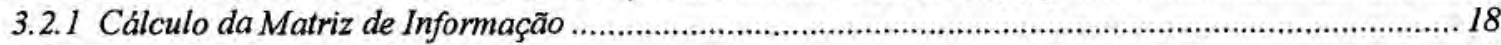

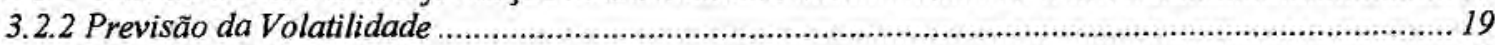

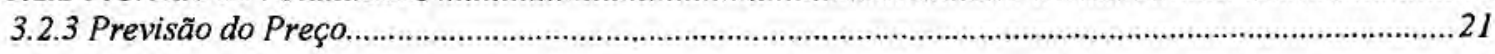

CAPÍTULO 4: INFERÊNCIA BAYESIANA PARA OS MODELOS ARCH(P) E GARCH(P,Q)............ 23

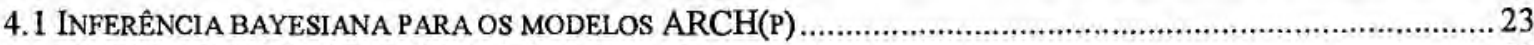

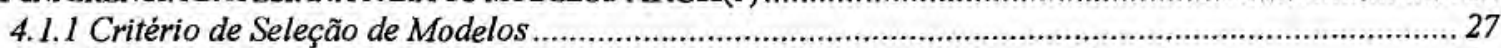

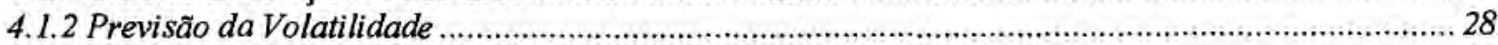

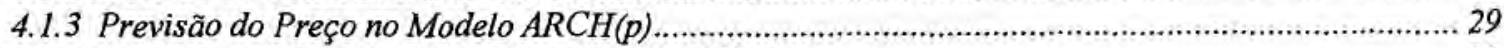

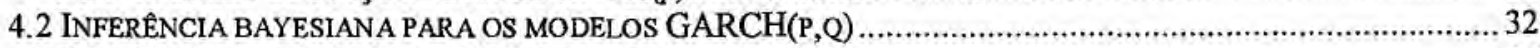

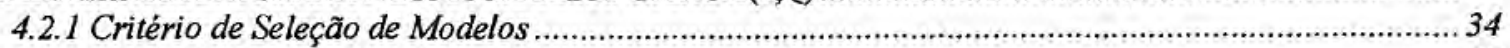

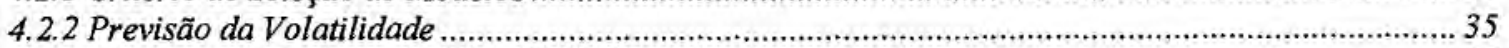

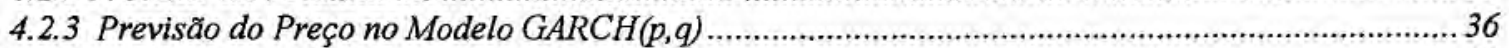

4.3 AVALIAÇÃO DA PREVISÃO UM PASSO À FRENTE

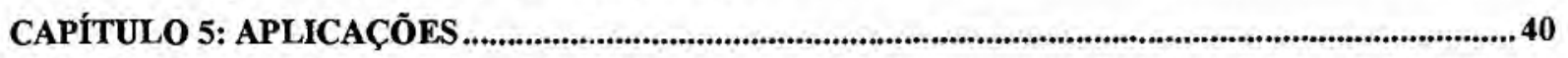

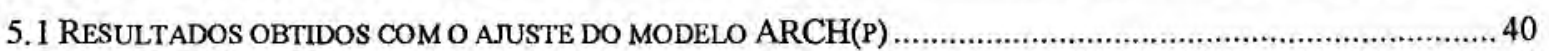

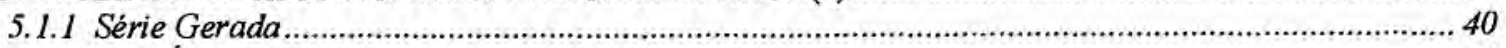

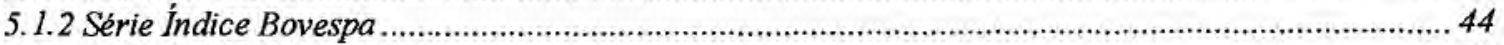

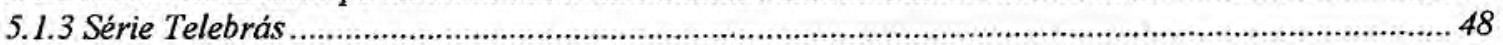

5.1.4 Série Cotação em Dólar Americano da moeda Iene Japonês............................................................52

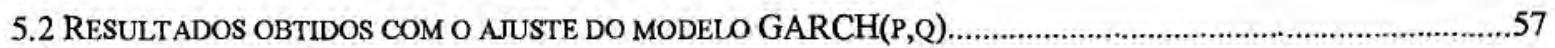

5.2.1 Série Gerada

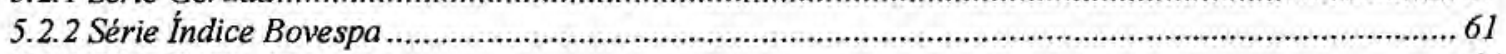

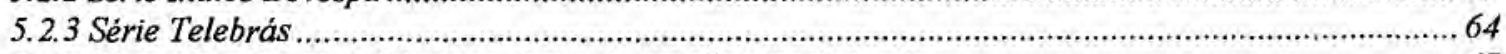

5.2.4 Série Cotação em Dólar Americano da moeda Iene Japonês .........................................................67

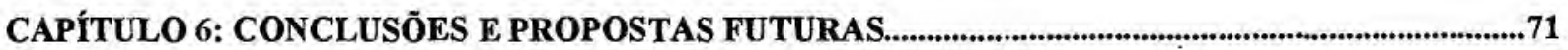

BIBLIOGRAFIA

72 


\section{Capítulo 1}

\section{Introdução}

A evolução do mercado financeiro tem exigido ferramentas de análise cada vez mais sofisticadas e complexas.

Uma simples análise gráfica de séries financeiras revela a presença de uma volatilidade alta para alguns períodos de tempo. Estas séries geralmente não são estacionárias na média e por isso é necessário transformações para obtermos estacionariedade. Uma transformação muito utilizada em séries financeiras é a taxa de retorno ou simplesmente retorno, que definimos a seguir.

Seja $P_{\imath}$ o preço de um determinado ativo o instante $t$. Os retornos podem ser definidos como:

$$
Y_{t}=\ln \left(P_{t} / P_{t-1}\right)=\ln \left(P_{t}\right)-\ln \left(P_{t-1}\right)
$$

As variações de um dia para outro costumam ser pequenas e por isso a razão $P_{t} / P_{t-1}$ oscila em torno de 1. Tomando-se o logaritmo desta razão, em geral, obtemos uma distribuição aproximadamente simétrica em torno de zero. Se considerarmos $\ln (1+\Delta) \cong \Delta$ para $\Delta$ pequeno, então o retorno definido desta forma é aproximadamente a variação relativa do preço dos ativos analisados.

A característica de interesse nestas séries de retornos é a volatilidade ou a variabilidade dos retornos.

A volatilidade está diretamente associada à variabilidade dos preços de um determinado ativo. Se os preços variam muito dizemos que este ativo é muito volátil. Usualmente utilizamos a variância ou o desvio-padrão como uma medida da volatilidade.

A estimação e previsão da volatilidade são fundamentais tanto para quantificar o risco de um determinado ativo como para a precificação de produtos financeiros.

Os modelos mais utilizados para descrever a volatilidade dos retornos são os modelos 
Auto-Regressivos com heterocedasticidade - $\mathrm{ARCH}$, proposto por Engle (1982), e sua extensão, os modelos ARCH-Generalizado (GARCH), proposto por Bollerslev (1986). Uma ampla revisão das propriedades desses modelos pode ser encontrada em Bollerslev et al (1992) e Lee (1992). Outros modelos definidos como exponencial GARCH (EGARCH) foram desenvolvidos por Nelson (1991) para considerar efeitos de assimetria na variância do processo.

A determinação de estimadores de máxima verossimilhança dos parâmetros dos modelos ARCH e GARCH exige a maximização de uma função de verossimilhança não linear. Portanto, as estimativas de máxima verossimilhança só podem ser obtidas numericamente. Engle (1982) sugere o uso do método de Newton como um método iterativo do cálculo das estimativas de máxima verossimilhança.

Muitos procedimentos para identificação, ajuste e diagnóstico dos modelos, assim como previsão de valores futuros de séries econométricas usam propriedades da teoria assintótica. Como estes modelos é muito distante da linearidade, qualquer uma dessas abordagens torna-se analiticamente intratável. Uma alternativa para contornar essas dificuldades é considerar a abordagem bayesiana dos modelos com heterocedasticidade.

A abordagem bayesiana para a classe de modelos ARCH foi tratada em Geweke (1989) para um caso particular de parametrização que permitiu a utilização de densidades a priori não informativas. As estimativas dos parâmetros foram obtidas usando algoritmo de simulação de Monte Carlo.

Uma extensão para os modelos ARMA-GARCH foi proposta em Nakatsuma (2000) utilizando densidades a priori normais para os parâmetros e algoritmo Metropolis-Hastings. Outra abordagem bayesiana dada em Migon et al (1999) reformula os modelos GARCH dentro da classe de modelos dinâmicos.

Neste trabalho apresentamos como os modelos ARCH e GARCH podem ser formulados na classe de modelos bayesianos e apresentamos técnicas de inferência, seleção de modelos e previsão de valores futuros das séries utilizando técnicas de simulação de Monte Carlo em Cadeia de Markov. A estimação bayesiana dos parâmetros foi obtida utilizando algoritmo Metropolis-Hastings e a previsão de valores futuros foi calculada por integração de Monte Carlo da densidade preditiva.

Os resultados obtidos na abordagem bayesiana foram comparados com os obtidos por máxima verossimilhança. 
Este trabalho está organizado do seguinte modo:

No capítulo 2 destacamos os principais fatos teóricos associados aos modelos ARCH e GARCH. As estimativas de máxima verossimilhança bem como as previsões da volatilidade e os critérios de seleção de modelos estão apresentados no capítulo 3.

No capítulo 4 apresentamos a abordagem bayesiana para a classe de modelos ARCHGARCH, a previsão da volatilidade para estes modelos e descrevemos o critério da densidade preditiva ordenada utilizado para selecionar o melhor modelo.

Por fim, no capitulo 5, aplicamos os métodos de máxima verossimilhança e bayesiano em séries geradas por modelos $\operatorname{ARCH}(2)$ e $\operatorname{GARCH}(1,1)$. Também foram utilizadas séries reais do Índice Bovespa, da Telebrás e da Cotação em Dólar Americano da moeda Iene Japonês. Comparamos os resultados obtidos pelos 2 métodos.

No capítulo 6 discutimos as conclusões obtidas neste trabalho e apresentamos algumas propostas futuras. 


\section{Capítulo 2}

\section{Modelos ARCH e GARCH}

\subsection{Modelo ARCH(p)}

Muitos modelos têm sido propostos e empregados na literatura financeira e econométrica para caracterizar o comportamento da volatilidade do retorno de ativos.

Uma classe de modelos muito utilizada é a dos modelos Auto-Regressivos com heterocedasticidade(ARCH) proposto por Engle (1982) e suas extensões, especialmente o ARCH-Generalizado(GARCH), introduzido por Bollerslev (1986).

$\mathrm{O}$ modelo linear $\mathrm{ARCH}(\mathrm{p})$ proposto por Engle pode ser resumido em:

$$
\begin{aligned}
& y_{t}=\mathbf{x}_{t} \boldsymbol{\beta}+z_{t} \\
& z_{\mathrm{t}} \mid \Omega_{t-1} \sim P\left(0, h_{t}\right) \\
& h_{t}=\alpha_{0}+\sum_{j=1}^{p} \alpha_{j} z_{t-j}^{2}
\end{aligned}
$$

onde $P(\cdot)$ é uma distribuição paramétrica, usualmente a Normal ou $t$-Student, $\mathbf{x}_{t}$ representa um vetor de variáveis exógenas, $\beta$ é um vetor de parâmetros desconhecidos e $\Omega_{t-1}$ representa o conjunto de informações disponiveis até o instante $t-1$.

Neste trabalho consideramos os modelos onde $\beta=0$ e o processo $z_{t}$ satisfazendo 0 modelo

$$
\begin{gathered}
z_{t}=h_{t}^{1 / 2} \varepsilon_{t} \\
h_{t}=\alpha_{0}+\sum_{j=1}^{p} \alpha_{j} z_{t-j}^{2}
\end{gathered}
$$

onde $\left\{\varepsilon_{t}, t \geq 0\right\}$ é um ruído branco i.i.d. $N(0,1)$. 
Por definição, $z_{t}$ é serialmente não correlacionada com média zero mas com variância condicional igual a $h_{t}$ que pode estar mudando através do tempo, ou seja, $z_{t} \mid \Omega_{t-1} \sim N\left(0, h_{t}\right)$.

No modelo $\operatorname{ARCH}(\mathrm{p})$ a variância condicional $h_{t}$ é função dos valores passados ao quadrado do processo. Para assegurar que a variância condicional seja estritamente positiva para todas as realizações de $z_{t}$, os parâmetros em (2.5) devem ser restritos a $\alpha_{0}>0$ e $\alpha_{j} \geq 0$ para $j=1, \ldots, p$.

Através da lei das esperanças iterativas pode ser mostrado que a variância incondicional de um $\mathrm{ARCH}(\mathrm{p})$ é dada por:

$$
E\left(z_{t}^{2}\right)=\frac{\alpha_{0}}{1-\sum_{j=1}^{p} \alpha_{j}}
$$

Para que o processo tenha covariância estacionária é necessário que $\sum_{j=1}^{p} \alpha_{j}<1$.

Caso Particular: Quando $\mathrm{p}=1$ temos o modelo $\mathrm{ARCH}(1)$ :

$$
h_{t}=\alpha_{0}+\alpha_{1} z_{t-1}^{2} \quad \alpha_{0}>0, \alpha_{1} \geq 0
$$

Engle (1982) mostrou que o segundo e o quarto momento de um processo gerado por um modelo $\operatorname{ARCH}(1)$ são definidos como $E\left(z_{t}^{2}\right)=\frac{\alpha_{0}}{1-\alpha_{1}}$ e $E\left(z_{t}^{4}\right)=\frac{3 \alpha_{0}^{2}}{\left(1-\alpha_{1}\right)^{2}} \frac{1-\alpha_{1}^{2}}{1-3 \alpha_{1}^{2}}$, para $\alpha_{1}<1$ e $3 \alpha_{1}^{2}<1$, respectivamente. Isto tem duas implicações. Primeiro, embora o modelo condicional seja gaussiano, a distribuição incondicional tem caudas mais "cheias" quando comparadas com a Normal. Isto acontece porque $\frac{1-\alpha_{1}^{2}}{1-3 \alpha_{1}^{2}}>1$. Sob uma distribuição incondicional gaussiana o coeficiente de curtose é dado por $\frac{3 \alpha_{0}^{2}}{\left(1-\alpha_{1}\right)^{2}}$, conseqüentemente menor que $\frac{3 \alpha_{0}^{2}}{\left(1-\alpha_{1}\right)^{2}} \frac{1-\alpha_{1}^{2}}{1-3 \alpha_{1}^{2}}$. Segundo, embora a distribuição condicional seja 
heterocedástica, a distribuição incondicional é homocedástica.

Algumas representações alternativas de (2.5) podem ser utilizadas para identificar a ordem $(p)$ do modelo ARCH. Para se entender melhor a dinâmica do modelo ARCH(p), Harvey (1992) considerou a seguinte relação:

$$
z_{t}^{2}=h_{t}+\left(z_{t}^{2}-h_{t}\right)
$$

onde $h_{t}=\alpha_{0}+\sum_{j=1}^{p} \alpha_{j} z_{t-j}^{2}$ e $z_{t}=h_{t}^{1 / 2} \varepsilon_{t}$.

Portanto,

$$
z_{t}^{2}=\alpha_{0}+\sum_{j=1}^{p} \alpha_{j} z_{t-j}^{2}+\left(h_{t} \varepsilon_{t}^{2}-h_{t}\right)
$$

Chamando $v_{t}=h_{t}\left(\varepsilon_{t}^{2}-1\right)$, a equação (2.9) pode ser reescrita como:

$$
z_{t}^{2}=\alpha_{0}+\sum_{j=1}^{p} \alpha_{j} z_{t-j}^{2}+v_{t}
$$

que é um modelo $\operatorname{AR}(\mathrm{p})$ para $z_{t}^{2}$ com um ruído $v_{t}$. Portanto, a identificação da ordem do modelo $\mathrm{ARCH}(\mathrm{p})$ pode ser baseada na função de autocorrelação e autocorrelação parcial do processo ao quadrado. No entanto, a utilização do modelo (2.10) para inferência dos parâmetros do modelo $\mathrm{ARCH}(\mathrm{p})$ é impraticável uma vez que não se conhece a distribuição do ruído $v_{t}$, o que dificulta a obtenção da função de verossimilhança para este modelo.

\subsection{Modelo GARCH(p,q)}

Para caracterizar completamente o comportamento de $h_{t}$ é muitas vezes necessário a utilização de muitos valores passados do processo. A estimação de uma estrutura totalmente livre para (2.5) freqüentemente viola as restrições de não negatividade imposta para os $\alpha_{j}$, $j=1, \ldots, p$. Para resolver este problema associado aos modelos ARCH, Bollerslev (1986) sugeriu uma estrutura alternativa e mais flexível dada pelo ARCH-Generalizado, ou 
$\operatorname{GARCH}(\mathrm{p}, \mathrm{q})$ :

$$
\begin{gathered}
z_{t} \mid \Omega_{t-1} \sim N\left(0, h_{t}\right) \\
h_{t}=\alpha_{0}+\sum_{i=1}^{p} \alpha_{i} z_{t-i}^{2}+\sum_{j=1}^{q} \beta_{j} h_{t-j} \\
=\alpha_{0}+A(L) z_{t}^{2}+B(L) h_{t}
\end{gathered}
$$

onde $p>0, q \geq 0, \alpha_{0}>0, \alpha_{i} \geq 0 i=1,2, \ldots, p, \beta_{j} \geq 0, j=1,2, \ldots, q$. Quando $q=0$ o processo se reduz a um $\operatorname{ARCH}(p)$. As restrições são impostas aos parâmetros para assegurar que a variância seja estritamente positiva.

Definindo o conjunto de informações passadas por $\Omega_{t-1}=\left\{z_{1}, \ldots, z_{t-1}\right\}$, a distribuição condicional do processo $z_{t}$ é normal com média zero e variância $h_{t}$, isto é, $z_{t} \mid \Omega_{t-1} \sim N\left(0, h_{t}\right)$.

$\mathrm{O}$ modelo $\mathrm{GARCH}(\mathrm{p}, \mathrm{q})$ definido em (2.11) é estacionário no sentido amplo com $E\left(z_{t}\right)=0, \operatorname{Var}\left(z_{t}\right)=\frac{\alpha_{0}}{1-\sum_{i=1}^{p} \alpha_{i}-\sum_{j=1}^{q} \beta_{j}} \operatorname{ecov}\left(z_{t}, z_{s}\right)=0$ para $t \neq s$ se e somente se $\sum_{i=1}^{p} \alpha_{i}+\sum_{j=1}^{q} \beta_{j}<1$

Caso Particular: Quando $\mathrm{p}=\mathrm{q}=1$ temos o modelo $\mathrm{GARCH}(1,1)$ e o modelo (2.11) é reduzido a:

$$
h_{t}=\alpha_{0}+\alpha_{1} z_{t-1}^{2}+\beta_{1} h_{t-1} \quad \alpha_{0}>0, \alpha_{1} \geq 0 \mathrm{e} \beta_{1} \geq 0
$$

Pode ser mostrado que o modelo $\operatorname{GARCH}(\mathrm{p}, \mathrm{q})$ gera uma distribuição incondicional leptocúrtica para $z_{t}$. Por exemplo, Bollerslev (1986) mostrou que o quarto momento de um modelo GARCH(1,1) é dado por:

$$
E\left(z_{t}^{4}\right)=\frac{3 \alpha_{0}^{2}\left(1+\alpha_{1}+\beta_{1}\right)}{\left[\left(1-\alpha_{1}-\beta_{1}\right)\left(1-\beta_{1}^{2}-2 \alpha_{1} \beta_{1}-3 \alpha_{1}^{2}\right)\right]}
$$


que é maior que o de uma variável aleatória normal se $\alpha_{1}>0$ e $\beta_{1}>0$.

Uma representação equivalente dos modelos $\operatorname{GARCH}(p, q)$ é dada por:

$$
z_{t}^{2}=\alpha_{0}+\sum_{i=1}^{p} \alpha_{i} z_{t-i}^{2}+\sum_{j=1}^{q} \beta_{j} z_{t-j}^{2}-\sum_{j=1}^{q} \beta_{j} w_{t-j}+w_{t}
$$

e

$$
w_{t}=z_{t}^{2}-h_{t}=h_{t}\left(\varepsilon_{t}^{2}-1\right)
$$

onde $\varepsilon_{t} \sim$ i.i.d $N(0,1)$.

Note que, por definição, $w_{t}$ é um ruído branco, ou seja, serialmente não correlacionado com média zero. Portanto, o processo $z_{t}^{2}$ pode ser interpretado como um processo $A R M A$ de ordem $m=\max \{p, q\}$ e $q$ respectivamente. Apesar do modelo ARMA apresentado em (2.14) ser mais estudado teoricamente, o modelo GARCH é mais prático de se trabalhar. Como no caso dos modelos $\mathrm{ARCH}(\mathrm{p})$, a representação (2.14) pode ser utilizada para identificação preliminar da ordem do modelo $\operatorname{GARCH}(p, q)$. 


\section{Capítulo 3}

\section{Estimadores de máxima verossimilhança dos modelos ARCH(p) e GARCH(p,q)}

\subsection{Estimadores de máxima verossimilhança dos modelos $A R C H(p)$}

Dada uma trajetória observada $\mathbf{Z}=\left\{z_{t}, t=1,2, \ldots, T\right\}$ do processo $z_{t}$, a função de verossimilhança para $z_{t}, t=p+1, \ldots, T$ é dada por:

$$
L(\boldsymbol{\alpha} \mid \mathbf{Z}) \propto \prod_{t=p+1}^{T}\left(\frac{1}{h_{t}}\right)^{1 / 2} \exp \left\{-\frac{z_{t}^{2}}{2 h_{t}}\right\}
$$

onde $\boldsymbol{\alpha}=\left(\alpha_{0}, \alpha_{1}, \ldots, \alpha_{p}\right)$. Denotamos a média do log da função de verossimilhança por:

$$
l(\boldsymbol{\alpha} \mid \mathbf{z})=\frac{1}{T} \sum_{t=p+1}^{T} l_{t}(\mathbf{\alpha} \mid \mathbf{z})
$$

onde $l_{t}(\boldsymbol{\alpha} \mid \mathbf{Z})$ indica o log da verossimilhança da $t$-ésima observação, ou seja,

$$
l_{t}\left(\alpha \mid z_{t}\right)=-\frac{1}{2} \log h_{t}-\frac{z_{t}^{2}}{2 h_{t}}
$$

O gradiente da função $l(\alpha \mid \mathbf{z})$ é dado por:

$$
\frac{\partial l}{\partial \boldsymbol{\alpha}}=\frac{1}{T} \sum_{t=p+1}^{T} \frac{\partial l_{t}}{\partial \boldsymbol{\alpha}}
$$


onde $\frac{\partial l_{t}}{\partial \boldsymbol{\alpha}}$ é o vetor $1 \times(p+1)$ cujas componentes são:

$$
\frac{\partial l_{t}}{\partial \alpha_{j}}=\frac{1}{2 h_{t}}\left(\frac{\partial h_{t}}{\partial \alpha_{j}}\right)\left(\frac{z_{t}^{2}}{h_{t}}-1\right) \quad j=0,1, \ldots, p
$$

Da equação (2.5) temos:

$$
\frac{\partial h_{i}}{\partial \alpha_{j}}= \begin{cases}1 & j=0 \\ z_{t-j}^{2} & j=1,2, \ldots, p, t \geq p+1\end{cases}
$$

Portanto,

$$
\frac{\partial l_{t}}{\partial \boldsymbol{\alpha}}=\frac{1}{2 h_{t}} v_{t}\left(\frac{z_{t}^{2}}{h_{t}}-1\right)
$$

onde $v_{t}=\left(1, z_{t-1}^{2}, \ldots, z_{t-p}^{2}\right)$ e o gradiente de $l(\boldsymbol{\alpha} \mid \mathbf{z})$ pode ser calculado substituindo (3.7) em (3.4), resultando em:

$$
\frac{\partial l}{\partial \boldsymbol{\alpha}}=\frac{1}{T} \sum_{t=p+1}^{T} \frac{1}{2 h_{t}} v_{t}\left(\frac{z_{t}^{2}}{h_{t}}-1\right)
$$

Denotando por:

$$
\begin{aligned}
\tilde{v}_{t}=\left(1, z_{t-1}^{2}, \ldots, z_{t-p}^{2}\right) / h_{t} & ; t=p+1, \ldots, T \\
\tilde{v}^{\prime}=\left(\tilde{v}_{p+1}^{\prime}, \ldots, \tilde{v}_{T}^{\prime}\right) & \\
f_{t}=\left(\frac{z_{t}^{2}}{h_{t}}-1\right) & \\
f^{\prime}=\left(f_{p+1}, \ldots, f_{T}\right) &
\end{aligned}
$$

podemos escrever $\frac{\partial l}{\partial \boldsymbol{\alpha}}$ na forma matricial como:

$$
\frac{\partial l}{\partial \alpha}=\frac{1}{2 T} \tilde{v}^{\prime} f
$$




\subsubsection{Cálculo da Matriz de Informação}

Denotando a matriz Hessiana de $l(\boldsymbol{\alpha} \mid \mathbf{Z})$ por $H=\left\{\frac{\partial^{2} l}{\partial \alpha_{i} \partial \alpha_{j}}, i, j=0,1, \ldots, p\right\}$ temos:

$$
\frac{\partial^{2} l}{\partial \alpha_{i} \partial \alpha_{j}}=\frac{1}{T} \sum_{t=p+1}^{T} \frac{\partial^{2} l_{t}}{\partial \alpha_{i} \partial \alpha_{j}}
$$

onde

$$
\begin{gathered}
\frac{\partial^{2} l_{t}}{\partial \alpha_{i} \partial \alpha_{j}}=\frac{\partial}{\partial \alpha_{i}}\left[\frac{1}{2 h_{t}} \frac{\partial h_{t}}{\partial \alpha_{j}}\left(\frac{z_{t}^{2}}{h_{t}}-1\right)\right] \\
\frac{\partial^{2} l_{t}}{\partial \alpha_{i} \partial \alpha_{j}}=-\frac{1}{2 h_{t}^{2}}\left(\frac{\partial h_{t}}{\partial \alpha_{i}}\right)\left(\frac{\partial h_{t}}{\partial \alpha_{j}}\right)\left(\frac{z_{t}^{2}}{h_{t}}-1\right)+\frac{1}{2 h_{t}} \frac{\partial^{2} h_{t}}{\partial \alpha_{i} \partial \alpha_{j}}\left(\frac{z_{t}^{2}}{h_{t}}-1\right)+\frac{1}{2 h_{t}}\left(\frac{\partial h_{t}}{\partial \alpha_{j}}\right)\left(-\frac{z_{t}^{2}}{h_{t}^{2}} \frac{\partial h_{t}}{\partial \alpha_{i}}\right)
\end{gathered}
$$

Da equação (2.5) temos que $\frac{\partial^{2} h_{t}}{\partial \alpha_{i} \partial \alpha_{j}}=0$, assim (3.12) pode ser simplificada para:

$$
\frac{\partial^{2} l_{t}}{\partial \alpha_{i} \partial \alpha_{j}}=-\frac{1}{2 h_{t}^{2}}\left(\frac{\partial h_{t}}{\partial \alpha_{i}}\right)\left(\frac{\partial h_{t}}{\partial \alpha_{j}}\right)\left(2 \frac{z_{t}^{2}}{h_{t}}-1\right)
$$

A matriz de informação $I=\left\{I_{i j}, i, j=0,1, \ldots p\right\}$ é definida como:

$$
I_{i j}=-E\left[\frac{1}{T} \sum_{t=p+1}^{T} \frac{\partial^{2} l_{t}}{\partial \alpha_{i} \partial \alpha_{j}}\right] \quad i, j=0,1, \ldots, p
$$

Usando $\circ$ fato que $E\left(2 \frac{z_{t}^{2}}{h_{t}}-1\right)=2 \frac{E\left(z_{t}^{2}\right)}{h_{t}}-1=1$, a matriz de informação $\hat{I}=\left\{\hat{I}_{i j}, i, j=0,1, \ldots p\right\}$ pode ser consistentemente estimada por: 


$$
\hat{I}=\frac{1}{T} \sum_{t=p+1}^{T} \frac{1}{2 h_{t}^{2}}\left\{\frac{\partial h_{t}}{\partial \alpha_{i}} \frac{\partial h_{t}}{\partial \alpha_{j}}\right\}
$$

com $i, j=0,1, \ldots, p$. Usando a definição $\widetilde{v}_{t}=\left(1, z_{t-1}^{2}, \ldots, z_{t-p}^{2}\right) / h_{t}, t=p+1, \ldots, T$ temos:

$$
\hat{I}=\frac{1}{2 T} \sum_{t=p+1}^{T} \widetilde{v}_{t}^{\prime} \widetilde{v}_{t}
$$

e sendo $\widetilde{v}^{\prime}=\left(\widetilde{v}_{p+1}^{\prime}, \ldots, \widetilde{v}_{T}^{\prime}\right)$ podemos escrever a matriz de informação por:

$$
\hat{I}=\frac{1}{2 T} \widetilde{v}^{\prime} \widetilde{v}
$$

Um método iterativo para calcular os estimadores de máxima verossimilhança para os parâmetros $\alpha_{j}, j=0,1, \ldots, p$ proposto em Engle (1982) consiste em interagir a seguinte equação recursiva:

$$
\boldsymbol{\alpha}^{(k+1)}=\boldsymbol{\alpha}^{(k)}+\left[\hat{I}^{(k)}\right]^{-1}\left[\frac{\partial l^{(k)}}{\partial \boldsymbol{\alpha}}\right]
$$

onde $\left[\hat{I}^{(k)}\right]$ é a matriz de informação em (3.17) e $\left[\partial l^{(k)} / \partial \alpha\right]$ o gradiente em (3.11), ambos avaliados com a estimativa $\boldsymbol{\alpha}^{(k)}$. Substituindo (3.11) e (3.17) em (3.18) temos:

$$
\boldsymbol{a}^{(k+1)}=\boldsymbol{\alpha}^{(k)}+\left[\tilde{v}^{\prime} \tilde{v}\right]^{-1} \widetilde{v}^{\prime} f
$$

Sob as condições em Engle (1982) pode ser estabelecido que o estimador de máxima verossimilhança $\hat{\boldsymbol{\alpha}}$ tem distribuição assintótica normal com distribuição limite

$$
\sqrt{T}(\hat{\boldsymbol{\alpha}}-\boldsymbol{\alpha}) \stackrel{D}{\rightarrow} N\left(0, I^{-1}\right)
$$




\subsubsection{Critério de Seleção de Modelos}

A identificação da ordem (p) dos modelos ARCH usando a equação (2.10) mostra-se imprecisa então faremos a identificação e seleção dos modelos baseados nos critérios de informação AIC (Akaike,1974) e BIC (Schwarz,1978). Estes critérios são dados por:

$$
\begin{gathered}
A I C=-\frac{2}{T} l(\boldsymbol{\alpha})+\frac{2 k}{T} \\
B I C=-\frac{2}{T} l(\boldsymbol{\alpha})+\frac{k \ln (T)}{T}
\end{gathered}
$$

onde $T$ é o número de observações na série $z_{t}$. Para séries com um número de observações $T$ muito grande, como é o caso de séries financeiras, o critério de Schwarz (BIC) mostra-se mais adequado que o AIC.

Os critérios AIC e BIC qualificam o modelo pelo chamado "critério da parcimônia" escolhendo o modelo pelo compromisso entre o ajuste adequado (máxima verossimilhança) e o menor número de parâmetros $k=p+1$. O modelo que apresenta o menor valor de AIC e BIC é o modelo mais adequado para representar os dados.

\subsubsection{Previsão da Volatilidade}

Usando a equação (2.5) e as observações $\mathbf{Z}^{T}=\left(z_{1}, \ldots, z_{T}\right)$ até o instante $T$ podemos calcular a previsão da variância condicional $k$-passos a frente de um modelo ARCH(p) por:

$$
\begin{aligned}
\hat{h}_{T+k} & =E\left[h_{T+k} \mid \mathbf{Z}^{T}\right] \\
& =\alpha_{0}+\sum_{j=1}^{p} \alpha_{j} E\left[z_{T+k-j}^{2} \mid \mathbf{Z}^{T}\right]
\end{aligned}
$$

Se $k \leq j$ os valores $z_{T+k-j} \in \mathbf{Z}^{T}$ então $E\left[z_{T+k-j}^{2} \mid \mathbf{Z}^{T}\right]=z_{T+k-j}^{2}$, caso contrário temos:

$$
E\left[z_{T+k-j}^{2} \mid \mathbf{Z}^{T}\right]=E\left[h_{T+k-j} \varepsilon_{T+k-j}^{2} \mid \mathbf{Z}^{T}\right], \quad j=1, \ldots, p ; k>j
$$


Como $h_{T+k-j}$ e $\varepsilon_{T+k-j}$ são independentes temos que $E\left[z_{T+k-j}^{2} \mid \mathbf{Z}^{T}\right]=E\left[h_{T+k-j} \mid \mathbf{Z}^{T}\right]$ pois $E\left[\varepsilon_{T+k-j}^{2} \mid \mathbf{Z}^{T}\right]=1$. Portanto, para $k>j$ :

$$
E\left[z_{T+k-j}^{2} \mid \mathbf{Z}^{T}\right]=E\left[h_{T+k-j} \mid \mathbf{Z}^{T}\right]
$$

Então:

$$
E\left[z_{T+k-j}^{2} \mid \mathbf{Z}^{T}\right]= \begin{cases}z_{T+k-j}^{2} & \text { se } k \leq j \\ \hat{h}_{T+k-j} & \text { se } k>j\end{cases}
$$

A equação (3.26) fornece as condições iniciais para o cálculo recursivo das previsões da volatilidade $\hat{h}_{T+k}, k$ passos a frente.

\subsubsection{Previsão do Preço}

\subsubsection{Previsão um passo à frente}

Vamos considerar a previsão um passo a frente de $y_{T+k}$ como sendo o valor esperado condicionado definido por:

$$
\hat{y}_{T+k}=E\left[y_{T+k} \mid \mathbf{Y}^{T+k-1}\right]
$$

onde $\mathbf{Y}^{T+k-1}=\left[y_{1}, \ldots, y_{T}, y_{T+1}, \ldots, y_{T+k-1}\right]$ é o vetor com todas as observações até o instante $T+k-1$. Usando a relação

$$
y_{T+k}=y_{T+k-1} e^{z_{T+k}}
$$

onde $z_{T+k} \sim N\left(0, h_{T+k}\right)$ e $h_{T+k}=\alpha_{0}+\sum_{j=1}^{p} \alpha_{j} z_{T+k-j}^{2}$ podemos calcular o valor esperado em (3.27) por:

$$
\hat{y}_{T+k}=E\left[y_{T+k-1} e^{z_{T+k}} \mid \mathbf{Y}^{T+k-1}\right]
$$


Sabendo que $y_{T+k-1} \in \mathbf{Y}^{T+k-1}$ e $z_{T+k} \sim N\left(0, h_{T+k}\right)$ e $h_{T+k}$ só depende de observações contidas em $\mathbf{Y}^{T+k-1}$ podemos calcular esse valor esperado por:

$$
\hat{y}_{T+k}=y_{T+k-1} e^{1 / 2 h_{T+k}}
$$

Portanto, a equação (3.30) pode ser usada recursivamente para fazer a previsão do preço $\hat{y}_{T+k}, k=1,2, \ldots$, um passo a frente.

\subsubsection{Previsão $k$ passos à frente}

Para fazer a previsão para $y_{T+k}$ quando as observações disponíveis são somente até o instante $T, \mathbf{Y}^{T}=\left[y_{1}, \ldots, y_{T}\right]$, vamos usar a fórmula recursiva:

$$
y_{T+k}=y_{T+k-1} e^{z_{T+k}}
$$

com a condição inicial $y_{T+1}=y_{T} e^{z_{T+1}}$. Assim, podemos escrever (3.31) como:

$$
y_{T+k}=y_{T} \exp \left(\sum_{i=1}^{k} z_{T+i}\right)
$$

Definindo a previsão para $y_{T+k}$ como:

$$
\hat{y}_{T+k}=E\left[y_{T+k} \mid \mathbf{Y}^{T}\right]
$$

temos:

$$
\hat{y}_{T+k}=E\left[y_{T} \exp \left(\sum_{i=1}^{k} z_{T+i}\right) \mid \mathbf{Y}^{T}\right]
$$

onde $\sum_{i=1}^{k} z_{T+1} \sim N\left(0, \sum_{i=1}^{k} h_{T+i}\right)$. 
A equação (3.34) pode ser escrita como:

$$
\begin{aligned}
E\left[y_{T} \exp \left(\sum_{i=1}^{k} z_{T+i}\right) \mid \mathbf{Y}^{T}\right] & =E_{\mathbf{z}^{*}}\left[E\left[y_{T} \exp \left(\sum_{i=1}^{k} z_{T+i}\right) \mid \mathbf{Y}^{T}, \mathbf{Z}^{*}\right]\right] \\
& =\mathrm{E}_{\mathbf{z}^{*}}\left[y_{T} \exp \left(\frac{1}{2} \sum_{i=1}^{k} h_{T+i}\left(\mathbf{z}^{*}\right)\right) \mid \mathbf{Y}^{T}\right]
\end{aligned}
$$

onde $\mathbf{Z}^{*}=\left[z_{T+1}, \ldots, z_{T+k-1}\right]$ e $h_{T+i}=\alpha_{0}+\sum_{j=1}^{p} \alpha_{j} z_{T+i-j}^{2}, i=1, \ldots, k$. Então, a previsão $k$ passos à frente é dada por:

$$
\hat{y}_{T+k}=y_{T} E_{\mathbf{z}} \cdot\left[\exp \left(\frac{1}{2} \sum_{i=1}^{k} h_{T+i}\left(\mathbf{z}^{*}\right)\right) \mid \mathbf{Y}^{T}\right]
$$

O valor esperado em (3.36) pode ser estimado por Monte Carlo gerando-se $\mathbf{Z}^{*} \sim N(0, \Sigma)$ onde $\Sigma=\operatorname{diag}\left(\hat{h}_{T+1}, \ldots, \hat{h}_{T+k-1}\right)$. Assim:

$$
\hat{y}_{T+k} \approx \frac{y_{T}}{M} \sum_{l=1}^{M} \exp \left(\frac{1}{2} \sum_{i=1}^{k} h_{T+i}^{(l)}\right)
$$

onde $h_{T+i}^{(l)}=\alpha_{0}+\sum_{j=1}^{p} \alpha_{j} z_{T+i-j}^{2(l)}, l=1, \ldots, M$ e $i=1, \ldots, k$

\subsection{Estimadores de máxima verossimilhança dos modelos $\mathrm{GARCH}(p, q)$}

$$
\operatorname{Seja} \widetilde{N}^{\widetilde{Z_{t}^{\prime}}}=\left(1, z_{t-1}^{2}, \ldots, z_{t-p}^{2}, h_{t-1}, \ldots, h_{t-q}\right) \text { e } \boldsymbol{\theta}^{\prime}=\left(\alpha_{0}, \alpha_{1}, \ldots, \alpha_{p}, \beta_{1}, \beta_{2}, \ldots, \beta_{q}\right) \text {. Vamos }
$$

denotar o modelo $\operatorname{GARCH}(\mathrm{p}, \mathrm{q})$ por:

$$
\begin{gathered}
z_{t} \mid \Omega_{t-1} \sim N\left(0, h_{t}\right) \\
h_{t}=\mathbf{Z}_{t}^{\prime} \boldsymbol{\theta}
\end{gathered}
$$

A média do $\log$ da função de verossimilhança para uma trajetória observada $\mathbf{Z}=\left\{z_{t}, t=1,2, \ldots T\right\}$ é dada por: 


$$
l(\boldsymbol{\theta} \mid \mathbf{Z})=\frac{1}{T} \sum_{t=m+1}^{T} l_{t}(\boldsymbol{\theta} \mid \mathbf{Z})
$$

onde $m=\max \{p, q\}$ e $l_{t}(\boldsymbol{\theta} \mid \mathbf{Z})$ é dado por:

$$
l_{t}\left(\theta \mid z_{t}\right)=-\frac{1}{2} \log h_{t}-\frac{z_{t}^{2}}{2 h_{t}}
$$

Derivando $l_{t}$ com relação aos parâmetros do modelo $\boldsymbol{\theta}^{\prime}=\left(\alpha_{0}, \alpha_{1}, \ldots, \alpha_{p}, \beta_{1}, \beta_{2}, \ldots, \beta_{q}\right)$ temos:

$$
\frac{\partial l_{t}}{\partial \theta}=\frac{1}{2 h_{t}}\left(\frac{\partial h_{t}}{\partial \theta}\right)\left(\frac{z_{t}^{2}}{h_{t}}-1\right)
$$

e da equação (3.39) temos que:

$$
\frac{\partial h_{t}}{\partial \theta}=\mathbf{Z}_{t}+\sum_{j=1}^{q} \beta_{j} \frac{\partial h_{t-j}}{\partial \theta}
$$

Aqui estamos usando a definição do vetor $\mathbf{Z}_{t}^{\prime}=\left(1, z_{t-1}^{2}, \ldots, z_{t-p}^{2}, h_{t-1}, \ldots, h_{t-q}\right)$. A única diferença no cálculo da derivada de $l_{t}$ entre os modelos $\operatorname{ARCH}(\mathrm{p})$ e $\operatorname{GARCH}(\mathrm{p}, \mathrm{q})$ é a inclusão do termo recursivo em (3.43). A propriedade de que $\sum_{j=1}^{q} \beta_{j}<1$ assegura que a equação recursiva (3.43) è convergente.

Denotando por:

$$
\begin{array}{rlr}
\widetilde{v}_{t} & =\left(\mathbf{Z}_{t}+\sum_{j=1}^{q} \beta_{j} \frac{\partial h_{t-j}}{\partial \boldsymbol{\theta}}\right) / h_{t} & ; t=m+1, \ldots, T \\
\widetilde{v}^{\prime}=\left(\widetilde{v}_{m+1}^{\prime}, \ldots, \widetilde{v}_{T}^{\prime}\right) & \\
f_{t}=\left(\frac{z_{t}^{2}}{h_{t}}-1\right) & ; t=m+1, \ldots, T \\
f^{\prime}=\left(f_{m+1}, \ldots, f_{T}\right) &
\end{array}
$$


Seja $\boldsymbol{\theta}^{(k)}$ o parâmetro estimado após a $k$-ésima iteração. A estimativa na $(k+1)$ iteração é calculada por:

$$
\boldsymbol{\theta}^{(k+1)}=\boldsymbol{\theta}^{(k)}+\lambda_{k}\left(\sum_{t=m+1}^{T} \frac{\partial l_{t}}{\partial \boldsymbol{\theta}} \frac{\partial l_{t}}{\partial \boldsymbol{\theta}^{\prime}}\right)^{-1} \sum_{t=m+1}^{T} \frac{\partial l_{t}}{\partial \boldsymbol{\theta}}
$$

onde $\lambda_{k}$ é escolhido de forma a maximizar a função de verossimilhança em uma dada direção. Neste trabalho $\lambda_{k}$ é calculado usando o método da razão áurea (Luenberger, 1984).

Para os estudos de casos realizados neste trabalho o estimador da matriz de covariância é:

$$
\mathfrak{J}^{-1}=-E\left[\frac{1}{T} \sum_{t=m+1}^{T}\left(\frac{\partial^{2} l_{t}(\boldsymbol{\theta})}{\partial \boldsymbol{\theta} \partial \boldsymbol{\theta}}\right)\right]^{-1}
$$

que não é definida positiva para as estimativas de máxima verossimilhança de $\boldsymbol{\theta}$.

Seguindo as recomendações de Bollerslev (1986) e Nakatsuma (2000) vamos considerar o estimador da matriz de covariância como $F^{-1}=T^{-1}\left(\sum_{t=m+1}^{T} \frac{\partial l_{t}}{\partial \boldsymbol{\theta}} \frac{\partial l_{t}}{\partial \boldsymbol{\theta}^{\prime}}\right)^{-1}$. Assim, pela teoria assintótica, podemos afirmar que:

$$
\sqrt{T}(\hat{\boldsymbol{\theta}}-\boldsymbol{\theta}) \stackrel{D}{\longrightarrow} N\left(0, F^{-1}\right)
$$

\subsubsection{Previsão da Volatilidade}

\subsubsection{Previsão um passo à frente}

Definindo os vetores $\boldsymbol{\alpha}=\left(\alpha_{1}, \ldots, \alpha_{p}\right), \quad \boldsymbol{\beta}=\left(\beta_{1}, \ldots, \beta_{q}\right), \quad \mathbf{Z}_{p}^{t-1}=\left(z_{t-1}^{2}, \ldots, z_{t-p}^{2}\right) \quad \mathrm{e}$ $\mathbf{h}_{q}^{t-1}=\left(h_{t-1}, \ldots, h_{t-q}\right)$ podemos escrever a volatilidade $h_{t}$ de um modelo GARCH(p,q) como:

$$
h_{t}=\alpha_{0}+\mathbf{Z}_{p}^{t-1} \boldsymbol{\alpha}^{\prime}+\mathbf{h}_{q}^{t-1} \boldsymbol{\beta}^{\prime}
$$


Através das observações disponíveis $\mathbf{Z}^{T+k-1}=\left[z_{1}, \ldots z_{T}, z_{T+1}, \ldots, z_{T+k-1}\right]$ até o instante $T+k-1$ podemos calcular a previsão da volatilidade um passo a frente por:

$$
\begin{aligned}
\hat{h}_{T+k} & =E\left[h_{T+k} \mid \mathbf{Z}^{T+k-1}\right] \\
& =\alpha_{0}+\mathbf{Z}_{p}^{T+k-1} \boldsymbol{\alpha}+\mathbf{h}_{q}^{T+k-1} \boldsymbol{\beta}^{\prime}
\end{aligned}
$$

onde os valores de $\mathbf{Z}_{p}^{T+k-1} \in \mathbf{Z}^{T+k-1}$ e $\mathbf{h}_{q}^{T+k-1}$ é calculada pela equação (3.52) com $h_{t}=E\left(z_{t}^{2}\right) \approx \frac{1}{T} \sum_{t=1}^{T} z_{t}^{2}, t<m+1$.

\subsubsection{Previsão $k$ passos à frente} dada por:

A previsão da variância condicional, $k$ passos à frente, de um modelo $\operatorname{GARCH}(\mathrm{p}, \mathrm{q})$ é

$$
\hat{h}_{T+k}=E\left[h_{T+k} \mid \mathbf{Z}^{T}\right]=\alpha_{0}+E\left[\mathbf{Z}_{p}^{T+k-1} \mid \mathbf{Z}^{T}\right] \mathbf{\alpha}^{\prime}+E\left[\mathbf{h}_{q}^{T+k-1} \mid \mathbf{Z}^{T}\right] \boldsymbol{\beta}^{\prime}
$$

onde $\mathbf{Z}^{T}$ são as observações disponíveis até o instante $T$ com

$$
E\left[\mathbf{Z}_{p}^{T+k-1} \mid \mathbf{Z}^{T}\right]= \begin{cases}\mathbf{Z}_{p}^{T+k-1} & \text { se } k \leq 1 \\ \hat{\mathbf{h}}_{q}^{T+k-1} & \text { se } k>1\end{cases}
$$

e

$$
E\left[\mathbf{h}_{q}^{T+k-1} \mid \mathbf{Z}^{T}\right]= \begin{cases}\mathbf{h}_{q}^{T+k-1} & \text { se } k \leq 1 \\ \hat{\mathbf{h}}_{q}^{T+k-1} & \text { se } k>1\end{cases}
$$

As equações (3.55) e (3.56) fornecem as condições iniciais para o cálculo recursivo das previsões da volatilidade $\hat{h}_{T+k}, k$ passos a frente. 


\subsubsection{Previsão do Preço}

\subsubsection{Previsão um passo à frente}

O cálculo da previsão do preço um passo à frente para os modelos $\operatorname{GARCH}(p, q)$ é feito de forma análoga ao dos modelos $\mathrm{ARCH}(\mathrm{p})$, ou seja,

$$
\hat{y}_{T+k}=E\left[y_{T+k} \mid \mathbf{Y}^{T+k-1}\right]
$$

$\operatorname{com} \mathbf{Y}^{T+k-1}=\left[y_{1}, \ldots, y_{T}, y_{T+1}, \ldots, y_{T+k-1}\right]$. Usando a relação

$$
y_{T+k}=y_{T+k-1} e^{z_{T+k}}
$$

onde $z_{T+k} \sim N\left(0, h_{T+k}\right), h_{T+k}=\alpha_{0}+\mathbf{Z}_{p}^{T+k-1} \boldsymbol{\alpha}^{\prime}+\mathbf{h}_{q}^{T+k-1} \boldsymbol{\beta}^{\prime}$ e sabendo que $y_{T+k-1} \in \mathbf{Y}^{T+k-1}$ e $h_{T+k}$ só depende de observações contidas em $\mathbf{Y}^{T+k-1}$ e dos valores das volatilidades passadas, com $h_{t}=\operatorname{var}\left(z_{t}\right) t<m+1$, podemos calcular (3.57) como:

$$
\begin{aligned}
\hat{y}_{T+k} & =E\left[y_{T+k-1} e^{z_{T+k}} \mid \mathbf{Y}^{T+k-1}\right] \\
& =y_{T+k-1} e^{1 / 2 h_{T+k}}
\end{aligned}
$$

Portanto, a equação (3.59) pode ser usada recursivamente para fazer a previsão do preço $\hat{y}_{T+k}, k=1,2, \ldots$, um passo a frente. A única diferença com relação a previsão de preços nos modelos $\mathrm{ARCH}(\mathrm{p})$ é o cálculo recursivo da volatilidade $h_{T+k}$ que aqui é feita usando a equação (3.53).

\subsubsection{Previsão $k$ passos à frente}

A previsão $k$ passos à frente também é feita da mesma maneira que a dos modelos $\mathrm{ARCH}(\mathrm{p})$. A diferença está no cálculo de $h_{T+i}$ que no caso dos modelos $\operatorname{GARCH}(\mathrm{p}, \mathrm{q})$ é calculada como $h_{T+i}=\alpha_{0}+\mathbf{Z}_{p}^{T+i-1} \boldsymbol{\alpha}^{\prime}+\mathbf{h}_{q}^{T+i-1} \boldsymbol{\beta}^{\prime}$. Portanto, a previsão $k$ passos à frente é dada por: 


$$
\hat{y}_{T+k}=y_{T} E_{\mathbf{z}^{*}}\left[\exp \left(\frac{1}{2} \sum_{i=1}^{k} h_{T+i}\left(\mathbf{z}^{*}\right)\right) \mid \mathbf{Y}^{T}\right]
$$

O valor esperado em (3.60) pode ser estimado por Monte Carlo gerando-se $\mathbf{Z}^{*} \sim N(0, \Sigma)$ onde $\Sigma=\operatorname{diag}\left(\hat{h}_{T+1}, \ldots, \hat{h}_{T+k-1}\right)$ e $\mathbf{Z}^{*}=\left[z_{T+1}, \ldots, z_{T+k-1}\right]$. Assim:

$$
\hat{y}_{T+k} \approx \frac{y_{T}}{M} \sum_{l=1}^{M} \exp \left(\frac{1}{2} \sum_{i=1}^{k} h_{T+i}^{(l)}\right)
$$

onde $h_{T+i}^{(l)}=\alpha_{0}+\mathbf{Z}_{p}^{T+i-1(l)} \boldsymbol{\alpha}^{\prime}+\mathbf{h}_{q}^{T+i-1(l)} \boldsymbol{\beta}^{\prime}, l=1, \ldots, M$ e $i=1, \ldots, k$. 


\section{Capítulo 4}

\section{Inferência bayesiana para os modelos $\mathrm{ARCH}(\mathrm{p})$ e $\operatorname{GARCH}(\mathbf{p}, \mathbf{q})$}

\subsection{Inferência bayesiana para os modelos $\mathrm{ARCH}(p)$}

Considerando uma trajetória observada dos retornos $\mathbf{Z}=\left(z_{1}, \ldots, z_{T}\right)$ a abordagem bayesiana do problema de inferência dos parâmetros do modelo $\mathrm{ARCH}(\mathrm{p})$ inicia-se combinando-se a função de verossimilhança dessa trajetória $L(\boldsymbol{\alpha} \mid \mathbf{Z})$ com a densidade a priori para os parâmetros $\pi_{0}(\boldsymbol{\alpha})$ resultando na densidade a posteriori dada por:

$$
\pi(\boldsymbol{\alpha} \mid \mathbf{Z}) \propto L(\boldsymbol{\alpha} \mid \mathbf{Z}) \pi_{0}(\boldsymbol{\alpha})
$$

No caso do modelo $\mathrm{ARCH}(\mathrm{p})$, os parâmetros estão restritos às condições $\alpha_{0}>0, \alpha_{j} \geq 0, j=1, \ldots, p$ e $\sum_{j=1}^{p} \alpha_{j}<1$ para assegurar covariância estacionária. Estas restrições nos permite, na abordagem bayesiana, propor densidades a priori uniformes para esses parâmetros, por exemplo, podemos propor densidades a priori para $\alpha_{j} \sim U(0,1) j=1,2, \ldots p$ uma vez que todos os $\alpha_{j}^{\prime}$ s devem ser menores que um. Também podemos considerar $\alpha_{0} \sim U\left(0, E\left(z_{t}^{2}\right)\right)$ dado que $E\left(z_{t}^{2}\right)=\alpha_{0} / 1-\sum_{j=1}^{p} \alpha_{j}$

Uma transformação que mapeia os domínios das densidades a priori uniformes $U\left(a_{j}, b_{j}\right)$ em um domínio $(-\infty,+\infty)$ pode apresentar vantagens quando se pretende usar algoritmos de MCMC. Assim, optamos por uma reparametrização dada por: 


$$
\phi_{j}=\log \left(\frac{\alpha_{j}-a_{j}}{b_{j}-\alpha_{j}}\right), \quad j=0,1,2, \ldots, p
$$

Com essa reparametrização a função de verossimilhança é escrita da mesma forma que em (3.1) mas com a volatilidade $h_{t}$ reescrita com os parâmetros $\alpha_{j}$ devidamente transformados em $\phi_{j}$ por (4.2), ou seja:

$$
L(\varphi \mid \mathbf{Z}) \propto \prod_{t=p+1}^{T}\left(\frac{1}{h_{t}}\right)^{1 / 2} \exp \left\{-\frac{z_{t}^{2}}{2 h_{t}}\right\}
$$

onde $\varphi=\left(\phi_{0}, \phi_{1}, \ldots, \phi_{p}\right)^{\prime}$.

As densidades a priori são definidas como:

$$
\pi_{j}\left(\phi_{j}\right) \sim \operatorname{Normal}\left(0, \sigma_{j}^{2}\right) \quad j=0,1,2, \ldots, p
$$

Com isto, através de (4.3) e (4.4) podemos escrever a densidade a posteriori como:

$$
\pi(\varphi \mid \mathbf{Z}) \propto L(\varphi \mid \mathbf{Z}) \prod_{j=0}^{p} \pi_{j}\left(\phi_{j}\right)
$$

Substituindo em (4.5) a equação (4.3) e as densidades a priori definidas em (4.4) obtemos:

$$
\pi(\boldsymbol{\varphi} \mid \mathbf{Z}) \propto[\operatorname{det}(\Sigma(\varphi))]^{-1 / 2} \exp \left\{-\frac{1}{2} Z^{\prime}\left[\Sigma^{-1}(\varphi)\right] Z\right\} \prod_{j=0}^{p} \pi_{j}\left(\phi_{j}\right)
$$

onde $\Sigma(\varphi)$ é a matriz diagonal dada por $\Sigma(\varphi)=\operatorname{diag}\left(h_{p+1}, \ldots, h_{T}\right)$ e denotando-se por:

$$
\psi(\varphi \mid \mathbf{Z})=[\operatorname{det}(\Sigma(\varphi))]^{-1 / 2} \exp \left\{-\frac{1}{2} Z^{\prime}\left[\Sigma^{-1}(\varphi)\right] Z\right\}
$$


podemos escrever

$$
\pi(\varphi \mid \mathbf{Z}) \propto \psi(\varphi \mid \mathbf{Z}) \prod_{j=0}^{p} \pi_{j}\left(\phi_{j}\right)
$$

A avaliação das densidades condicionais a posteriori pode ser feita por meio de métodos de simulação de Monte Carlo em Cadeia de Markov usando algoritmo MetropolisHastings (Chib \& Greenberg, 1995).

Considerando a posteriori conjunta dada em (4.8) obtemos as seguintes densidades condicionais a posteriori para $\phi_{j} j=0,1, \ldots, p$ :

$$
\Pi_{j}\left(\phi_{j} \mid \varphi_{-j}, \mathbf{Z}\right) \propto \psi(\varphi \mid \mathbf{Z}) \pi_{j}\left(\phi_{j}\right)
$$

onde $\pi_{j}\left(\phi_{j}\right), j=0,1, \ldots, p$ são as densidades a priori dada em (4.4), $\psi\left(\phi_{j}\right)$ tem a forma geral dada por (4.7) e $\varphi_{-j}$ é um vetor com os parâmetros do modelo exceto o parâmetro $\phi_{j}$, ou seja, $\varphi_{-j}=\left(\phi_{0}, \phi_{1}, \ldots, \phi_{j-1}, \phi_{j+1}, \ldots, \phi_{p}\right)^{\prime}$.

Nota: O uso de priori informativas como núcleo do algoritmo Metropolis-Hastings é apropriado sempre que tais densidades forem adequadamente escolhidas e o domínio dessas densidades envolverem o domínio da densidade a posteriori, como é o caso dos modelos $\operatorname{ARCH}(\mathrm{p})$.

Desta forma, o algoritmo M-H pode ser implementado com os seguintes passos:

\section{Algoritmo:}

1) Faça $i=1$ e forneça um valor inicial arbitrário $\varphi^{(0)}=\left(\phi_{j}^{(0)}\right) j=0,1 \ldots, p$.

2) Gere um novo valor $\beta$ a partir da densidade $\pi_{j}(\cdot)$.

3) Calcule a probabilidade de aceitação do novo valor gerado $\beta$ :

$$
\lambda\left(\phi_{j}^{(i-1)}, \beta\right)=\min \left\{1, \frac{\psi(\beta)}{\psi\left(\phi_{j}^{(i-1)}\right)}\right\}, \quad \psi\left(\phi_{j}^{(i-1)}\right) \neq 0
$$


4) Gere uma variável aleatória uniforme $u \sim U(0,1)$.

5) Faça $\phi_{j}^{(i)}=\left\{\begin{array}{l}\beta, \text { se } u \leq \lambda \\ \phi_{j}^{(i-1)}, \text { caso contrário }\end{array}\right\}$

6) Repetir 2 a 5 para $j=0, \ldots, p$.

7) Faça $i \leftarrow i+1$ e volte ao passo 2 ) até a convergência.

Após a convergência os parâmetros originais $\alpha_{j} j=0,1, \ldots, p$ podem ser recuperados pela transformação inversa:

$$
\alpha_{j}=\frac{b_{j} e^{\phi_{j}}+a_{j}}{1+e^{\phi_{j}}}
$$

No algoritmo Metropolis-Hastings utilizamos $a_{j}=0, j=0,1, \ldots, p, b_{0} \approx 1 / T \sum_{t=1}^{T} z_{t}^{2}$ e os demais $b_{j}=1, j=1, \ldots, p$.

O problema de inferência bayesiana pode ser expresso como a avaliação da esperança de uma função de interesse $g(\boldsymbol{\alpha})$ sob a densidade a posteriori $\pi(\boldsymbol{\alpha} \mid \mathbf{Z})$ :

$$
E[g(\boldsymbol{\alpha})]=\int_{\boldsymbol{\alpha}} g(\boldsymbol{\alpha}) \pi(\boldsymbol{\alpha} \mid \mathbf{Z}) d \boldsymbol{\alpha}
$$

A solução da integral múltipla em (4.11) não pode ser calculada analiticamente no presente caso.

Uma aproximação para (4.11) pelo Método de Monte Carlo pode ser obtida sempre que $E[g(\boldsymbol{\alpha})]$ existe, cuja precisão numérica pode ser avaliada. Para detalhes desse método ver Geweke (1986c).

Assim, seja $\left\{\boldsymbol{\alpha}^{(i)}\right\}$ uma seqüência i.i.d de um vetor aleatório $(p+1)$ dimensional com densidade conjunta $\pi(\boldsymbol{\alpha} \mid \mathbf{Z})$. Numericamente aproximamos $\bar{g} \equiv E[g(\boldsymbol{\alpha})]$ por:

$$
\bar{g}_{M}=\frac{1}{M} \sum_{i=1}^{M} g\left(\boldsymbol{\alpha}^{(i)}\right)
$$


Sob condições regulares para a aplicação do Teorema Central do Limite, ver Geweke (1986c), temos que $\bar{g}_{M} \rightarrow E[g(\boldsymbol{\alpha})]$ e pode ser mostrado que

$$
M^{1 / 2}\left(\bar{g}_{M}-\bar{g}\right) \Rightarrow N\left(0, \sigma^{2}\right)
$$

onde

$$
\sigma^{2}=E[g(\boldsymbol{\alpha})-\bar{g}]^{2}
$$

Se $\hat{\sigma}_{M}^{2}=\frac{1}{M-1} \sum_{i=1}^{M}\left(g\left(\boldsymbol{\alpha}^{(i)}\right)-\bar{g}_{M}\right)^{2}$ então $n \hat{\sigma}_{M}^{2} \rightarrow \sigma^{2}$. Nos referimos a $\hat{\sigma}_{M}$ como o erro padrão numérico. Então a precisão numérica pode ser assegurada sob a suposição $\bar{g}_{M} \sim N\left(\bar{g}, \hat{\sigma}_{M}^{2}\right)$ quando $M$ é grande.

Um resumo das principais estatísticas dos parâmetros $\alpha_{j}, j=0, \ldots, p$ podem ser obtidas pelo método de Monte Carlo dado por:

$$
E\left[g\left(\boldsymbol{\alpha}^{(i)}\right)\right]=\frac{1}{M} \sum_{i=1}^{M} g\left(\alpha_{j}^{(i)}\right)
$$

onde $M$ representa o tamanho da cadeia gerada com o algoritmo Metropolis-Hastings.

\subsubsection{Critério de Seleção de Modelos}

$\mathrm{Na}$ abordagem bayesiana utilizamos como critério para seleção dos modelos ajustados o critério da Densidade Preditiva Ordenada. Neste critério usamos a densidade $z_{T+k}$ condicionada aos dados $\mathbf{Z}$ e aos parâmetros $\boldsymbol{\alpha}=\left(\alpha_{0}, \alpha_{1}, \ldots, \alpha_{p}\right)^{\prime}$. Portanto, a densidade preditiva fica:

$$
\begin{gathered}
\pi\left(z_{T+k} \mid \mathbf{Z}\right)=\int_{\boldsymbol{\alpha}} \pi\left(z_{T+k} \mid \mathbf{Z}, \boldsymbol{\alpha}\right) \pi(\boldsymbol{\alpha} \mid \mathbf{Z}) d \boldsymbol{\alpha} \\
c_{k}=\pi\left(z_{T+k} \mid \mathbf{Z}\right)=\int_{\boldsymbol{\alpha}}\left(\frac{1}{2 \pi h_{T+k}}\right)^{1 / 2} \exp \left\{-\frac{1}{2} \frac{z_{T+k}^{2}}{h_{T+k}}\right\} \pi(\boldsymbol{\alpha} \mid \mathbf{Z}) d \boldsymbol{\alpha}
\end{gathered}
$$


onde $\pi(\boldsymbol{\alpha} \mid \mathbf{Z})$ é a densidade a posteriori para os parâmetros $\alpha_{0}, \alpha_{1}, \ldots, \alpha_{p}$ e a integral em (4.16) é uma integral múltipla no espaço dos parâmetros.

A equação (4.16) pode também ser avaliada usando as observações $\alpha_{j}^{(i)}, \quad j=$ $0,1, \ldots, p$ obtidas por simulação MCMC considerando $h_{T+k}$ como uma função dos parâmetros a. Neste caso, a estimativa de Monte Carlo da densidade preditiva é dada por:

$$
\hat{c}_{k}=\frac{1}{M} \sum_{i=1}^{M} \frac{1}{\sqrt{2 \pi h_{T+k}\left(\boldsymbol{\alpha}^{(i)}\right)}} \exp \left\{-\frac{1}{2} \frac{z_{T+k}^{2}}{h_{T+k}\left(\boldsymbol{\alpha}^{(i)}\right)}\right\}
$$

Um baixo valor de $\hat{c}_{k}$ indica que a observação $z_{T+k}$ é improvável para o modelo considerado. Assim, o critério de seleção de modelo baseado na densidade preditiva ordenada consiste em escolher o modelo $l$ que apresenta o maior valor de $\hat{c}(l)=\prod_{k=T+1}^{T+K} \hat{c}_{k}(l)$.

\subsubsection{Previsão da Volatilidade}

A previsão da variância condicional $k$ passos à frente de um modelo $\mathrm{ARCH}(\mathrm{p})$ é dada por:

$$
\hat{h}_{T+k}=E\left[h_{T+k} \mid \mathbf{Z}\right]
$$

$\mathrm{Na}$ abordagem bayesiana os parâmetros do modelo $\alpha=\left(\alpha_{0}, \alpha_{1}, \ldots, \alpha_{p}\right)$ são também variáveis aleatórias, portanto:

$$
\begin{aligned}
E\left[h_{T+k} \mid \mathbf{Z}\right] & =E_{\mathbf{\alpha}}\left[E\left[h_{T+k} \mid \mathbf{Z}, \boldsymbol{\alpha}\right]\right] \\
\hat{h}_{T+k} & =E_{\boldsymbol{\alpha}}\left[E\left[\alpha_{0}+\sum_{j=1}^{p} \alpha_{j} z_{T+k-j}^{2} \mid \mathbf{Z}, \boldsymbol{\alpha}\right]\right] \\
\hat{h}_{T+k} & =E_{\boldsymbol{\alpha}}\left[\alpha_{0}+\sum_{j=1}^{p} \alpha_{j} E\left[z_{T+k-j}^{2} \mid \mathbf{Z}\right]\right]
\end{aligned}
$$


Para $k \leq j$ os valores $z_{T+k-j} \in \mathbf{Z}$, caso contrário temos:

$$
\begin{aligned}
E\left[z_{T+k-j}^{2} \mid \mathbf{Z}\right] & =E\left[h_{T+k-j} \varepsilon_{T+k-j}^{2} \mid \mathbf{Z}\right] \\
\hat{h}_{T+k-j} & =E\left[h_{T+k-j} \mid \mathbf{Z}\right]
\end{aligned}
$$

Assim, a previsão $k$ passos à frente na abordagem bayesiana pode ser calculada por (4.19) considerando

$$
E\left[z_{T+k-j}^{2} \mid \mathbf{Z}\right]= \begin{cases}z_{T+k-j}^{2} & \text { se } k \leq j \\ \hat{h}_{T+k-j} & \text { se } k>j\end{cases}
$$

O valor esperado com relação a densidade a posteriori dos parâmetros $\boldsymbol{\alpha}$ pode ser aproximado pela estimativa de Monte Carlo considerando a amostra $\boldsymbol{\alpha}^{(i)}, i=1,2, \ldots, M$ gerada pelo algoritmo MCMC. Assim, temos:

$$
\hat{h}_{T+k}=\frac{1}{M} \sum_{i=1}^{M}\left(\alpha_{0}^{(i)}+\sum_{j=1}^{p} \alpha_{j}^{(i)} E\left[z_{T+k-j}^{2} \mid \mathbf{Z}\right]\right)
$$

\subsubsection{Previsão do Preço no Modelo ARCH(p)}

\subsubsection{Previsão um passo à frente}

A previsão um passo a frente dos preços $\left\{y_{t}, t=T+1, \ldots, T+k\right\}$ pode ser calculada usando-se a propriedade do valor esperado condicionado:

$$
\hat{y}_{T+k}=E_{\boldsymbol{\alpha}}\left[E\left[y_{T+k} \mid \mathbf{Y}^{T+k-1}, \boldsymbol{\alpha}\right]\right]
$$

Aqui estamos usando a mesma definição do caso clássico $\hat{y}_{T+k}=E\left[y_{T+k} \mid \mathbf{Y}^{T+k-1}\right]$ onde $\mathbf{Y}^{T+k-1}=\left[y_{1}, \ldots, y_{T}, y_{T+1}, \ldots, y_{T+k-1}\right]$ e $\boldsymbol{\alpha}=\left(\alpha_{0}, \alpha_{1}, \ldots, \alpha_{p}\right)$ é o vetor de parâmetros. 
Através da relação

$$
y_{T+k}=y_{T+k-1} e^{z_{T+k}}
$$

onde $z_{T+k} \sim N\left(0, h_{T+k}\right)$ e $h_{T+k}(\boldsymbol{\alpha})=\alpha_{0}+\sum_{j=1}^{p} \alpha_{j} z_{T+k-j}^{2}$ sendo escrita como função não só das observações passadas (supostas conhecidas) mas também do vetor de parâmetros do modelo que são considerados como um vetor aleatório $(p+1)$ dimensional podemos calcular o valor esperado condicional em (4.23) por:

$$
E\left[y_{T+k-1} e^{z_{T+k}} \mid \mathbf{Y}^{(T+k-1)}, \boldsymbol{\alpha}\right]=y_{T+k-1} \exp \left(1 / 2 h_{T+k(\alpha)}\right)
$$

Substituindo (4.25) em (4.23) temos:

$$
\hat{y}_{T+k}=E_{\alpha}\left[y_{T+k-1} \exp \left(1 / 2 h_{T+k(\alpha)}\right]\right.
$$

onde $y_{T+k-1}, y_{T+k-2, \cdots,} y_{1}$ são conhecidos no início do instante $T+k, k=1,2, \ldots, k$. Usando a amostra gerada pelo algoritmo de MCMC, $\alpha^{(i)}=\left(\alpha_{0}^{(i)}, \ldots, \alpha_{p}^{(i)}\right), i=1,2, \ldots, M$ podemos avaliar o valor esperado em (4.26) por:

$$
\hat{y}_{T+k}=\frac{1}{M} \sum_{i=1}^{M} y_{T+k-1} \exp \left(1 / 2 h_{T+k\left(\mathbf{\alpha}^{(i)}\right)}\right)
$$

onde $h_{T+k}\left(\boldsymbol{\alpha}^{(i)}\right)=\alpha_{0}^{(i)}+\sum_{j=1}^{p} \alpha_{j}^{(i)} z_{T+k-\jmath}^{2}$. A equação (4.27) pode ser usada para fazer a previsão um passo a frente para $k=1,2, \ldots, K$.

\subsubsection{Previsão $k$ passos à frente}

A previsão $k$ passos a frente pode ser calculada usando as observações $\mathbf{Y}^{(T)}=\left(y_{1}, \ldots, y_{T}\right)^{\prime}$ e uma função $\phi\left(\mathbf{Y}^{*}, \mathbf{Y}^{(T)}\right)$ das observações $\mathbf{Y}^{(T)}$ e de $k$ valores futuros 
$\mathbf{Y}^{*}=\left(y_{T+1}, \ldots, y_{T+k}\right)^{\prime} .0$ problema de previsão desses valores futuros consiste em calcular o valor esperado:

$$
E\left[\phi\left(\mathbf{Y}^{*}, \mathbf{Y}^{(T)}\right) \mid \mathbf{Y}^{(T)}\right]=E_{\alpha}\left[E\left[\phi\left(\mathbf{Y}^{*}, \mathbf{Y}^{(T)}\right) \mid \mathbf{Y}^{(T)}, \boldsymbol{\alpha}\right]\right]
$$

Considerando o vetor aleatório $\alpha$ de dimensão $(p+1)$ com densidade conjunta a posteriori $\pi\left(\boldsymbol{\alpha} \mid \mathbf{Y}^{(T)}\right)$ podemos calcular o valor esperado em (4.28) por:

$$
E\left[\phi\left(\mathbf{Y}^{*}, \mathbf{Y}^{(T)}\right) \mid \mathbf{Y}^{(T)}\right]=\int_{\boldsymbol{\alpha}} E\left[\phi\left(\mathbf{Y}^{*}, \mathbf{Y}^{(T)}\right) \mid \mathbf{Y}^{(T)}, \boldsymbol{\alpha}\right] \pi\left(\boldsymbol{\alpha} \mid \mathbf{Y}^{(T)}\right) d \boldsymbol{\alpha}
$$

Através da amostra gerada pelo algoritmo MCMC para o vetor $\alpha^{(i)}=\left(\alpha_{0}^{(i)}, \ldots, \alpha_{p}^{(i)}\right), i=1, \ldots, M$ podemos calcular (4.29) por:

$$
E\left[\phi\left(\mathbf{Y}^{*}, \mathbf{Y}^{(T)}\right) \mid \mathbf{Y}^{(T)}\right]=\frac{1}{M} \sum_{i=1}^{M} E\left[\phi\left(\mathbf{Y}^{*}, \mathbf{Y}^{(T)}\right) \mid \mathbf{Y}^{(T)}, \boldsymbol{\alpha}^{(i)}\right]
$$

Em alguns casos especiais, o valor esperado em (4.30) pode ser calculado analiticamente para um modelo $\mathrm{ARCH}(\mathrm{p})$. Vamos avaliar esse valor esperado gerando $\mathbf{y}^{*}$ das seguintes fórmulas recursivas:

(i) $h_{T+k}=\alpha_{0}+\sum_{j=1}^{p} \alpha_{j} z_{T+k-j}^{2}$

(ii) $z_{T+k} \sim N\left(0, h_{T+k}\right)$

(iii) $y_{T+k}=y_{T+k-1} e^{z_{T+k}}$

para $k=1,2, \ldots, K$. Dada a série gerada $y_{T+1}^{*}, \ldots, y_{T+k}^{*}, \phi\left(\mathbf{Y}^{*}, \mathbf{Y}^{(T)}\right)$ pode ser avaliada. Na implementação desse procedimento, $\boldsymbol{\alpha}^{(i)}$ é primeiro gerado da densidade $\pi\left(\boldsymbol{\alpha} \mid \mathbf{Y}^{(T)}\right)$ que é a densidade conjunta a posteriori. O vetor aleatório $\mathbf{Z}^{*}=\left(z_{T+1}, \ldots, z_{T+k}\right)^{\prime}$ é então construído e $\phi\left(\mathbf{Y}^{*}, \mathbf{Y}^{(T)}\right)$ é calculada. Esses procedimentos são repetidos $M$ vezes e a média de $\phi\left(\mathbf{Y}^{*}, \mathbf{Y}^{(T)}\right)$ pode então ser calculada. 


\subsection{Inferência bayesiana para os modelos $\operatorname{GARCH}(p, q)$}

A análise bayesiana feita para os modelos $\operatorname{GARCH}(\mathrm{p}, \mathrm{q})$ é similar a dos modelos $\operatorname{ARCH}(\mathrm{p})$. Consideramos densidades a priori uniformes para $\alpha_{i}$ e $\beta_{\mathrm{j}}$, ou seja, $\alpha_{i} \sim U(0,1)$ e $\beta_{j} \sim U(0,1), i=1, \ldots, p$ e $j=1, \ldots, q$ uma vez que $\sum_{i=1}^{p} \alpha_{i}+\sum_{j=1}^{q} \beta_{j}<1$. Também consideramos $\alpha_{0} \sim U\left(0, E\left(z_{t}^{2}\right)\right)$ dado que $E\left(z_{t}^{2}\right)=\frac{\alpha_{0}}{1-\sum_{i=1}^{p} \alpha_{i}-\sum_{j=1}^{q} \beta_{j}}$.

Como utilizamos algoritmos de MCMC optamos pela reparametrização dada em (4.2) que mapeia os domínios da densidade a priori uniforme $U\left(a_{i}, b_{i}\right)$ em um domínio $(-\infty,+\infty)$, ou seja, sendo $\boldsymbol{\theta}=\left(\alpha_{0}, \alpha_{1}, \ldots, \alpha_{p}, \beta_{1}, \ldots, \beta_{q}\right)^{\prime}$ o vetor de parâmetros, vamos escrever a reparametrização por:

$$
\phi_{i}=\log \left(\frac{\theta_{i}-a_{i}}{b_{i}-\theta_{i}}\right) \quad i=0,1, \ldots, p+q
$$

onde $\phi_{i}$ é uma componente do vetor de parâmetros transformados $\varphi=\left(\phi_{0}, \phi_{1}, \ldots, \phi_{p+q}\right)^{\prime}$.

Através da reparametrização (4.32) podemos rescrever a função de verossimilhança como:

$$
L(\boldsymbol{\varphi} \mid \mathbf{Z}) \propto \prod_{t=m+1}^{T}\left(\frac{1}{h_{t}}\right)^{1 / 2} \exp \left\{-\frac{z_{t}^{2}}{2 h_{t}}\right\}
$$

onde $h_{t}$ é a volatilidade definida em (2.11) com os parâmetros $\alpha_{i}, i=0, \ldots, p$ e $\beta_{\mathrm{j}}, j=1, \ldots, q$ transformados em $\phi_{i}$ e $m=\max (p, q)$.

As densidades a priori são definidas como:

$$
\pi_{i}\left(\phi_{i}\right) \sim \operatorname{Normal}\left(0, \sigma_{i}^{2}\right) \quad i=0,1,2, \ldots, p+q
$$


Com as densidades a priori definidas em (4.34) e a função de verossimilhança definida em (4.33) podemos escrever a densidade a posteriori como:

$$
\pi(\varphi \mid \mathbf{Z}) \propto L(\varphi \mid \mathbf{Z}) \prod_{i=0}^{p+q} \pi_{i}\left(\phi_{i}\right)
$$

A avaliação das densidades condicionais a posteriori foi feita por métodos de simulação de Monte Carlo em Cadeia de Markov utilizando o algoritmo Metropolis-Hastings (Chib \& Greenberg, 1995).

Através da posteriori conjunta obtida em (4.35) obtemos as seguintes densidades condicionais a posteriori para $\phi_{i}, i=0,1, \ldots, p+q$ :

$$
\pi_{i}\left(\varphi_{i} \mid \varphi_{-i}, \mathbf{Z}\right) \infty L(\varphi \mid \mathbf{Z}) \pi_{i}\left(\phi_{i}\right)
$$

onde $\pi_{i}\left(\phi_{i}\right), i=0,1, \ldots, p+q$ são as densidades a priori dada em (4.34), $L(\varphi \mid \mathbf{Z})$ é a função de verossimilhança definida em (4.33) e $\varphi_{-i}=\left(\phi_{0}, \phi_{1}, \ldots, \phi_{i-1}, \phi_{i+1}, \ldots, \phi_{p+q}\right)^{\prime}$.

Nota: Da mesma forma como foi justificado para os modelos $\mathrm{ARCH}(\mathrm{p})$, o uso de priori informativas como núcleo do algoritmo Metropolis-Hastings é apropriado sempre que tais densidades forem adequadamente escolhidas e o domínio dessas densidades envolverem o domínio da densidade a posteriori, como é o caso dos modelos $\operatorname{GARCH}(\mathrm{p}, \mathrm{q})$.

$\mathrm{O}$ algoritmo M-H utilizado está descrito na seção 4.1 .

Para os modelos GARCH(p,q), a função $\psi(\varphi \mid \mathbf{Z})$ só pode ser avaliada através de um método recursivo para o cálculo de $h_{t}$. Aqui, essa recursão é iniciada fazendo $h_{t} \approx T^{-1} \sum_{t=1}^{T} z_{t}^{2}, t<m+1$.

Após a convergência os parâmetros originais $\alpha_{i} i=0,1, \ldots, p$ e $\beta_{j} j=1, \ldots, q$ podem ser recuperados pela transformação inversa:

$$
\theta_{i}=\frac{b_{i} e^{\phi_{t}}+a_{i}}{1+e^{\phi_{i}}}
$$


As principais estatísticas dos parâmetros $\alpha_{i}, i=0, \ldots, p$ e $\beta_{\mathrm{j}}, j=1, \ldots, q$ podem ser obtidas pelo método de Monte Carlo dado por:

$$
\begin{aligned}
& E\left[g\left(\theta^{(l)}\right)\right]=\frac{1}{M} \sum_{l=1}^{M} g\left(\alpha_{i}^{(l)}\right) \\
& E\left[g\left(\theta^{(l)}\right)\right]=\frac{1}{M} \sum_{l=1}^{M} g\left(\beta_{j}^{(l)}\right)
\end{aligned}
$$

onde $M$ representa o tamanho da cadeia gerada com o algoritmo Metropolis-Hastings e $\boldsymbol{\theta}=\left(\alpha_{0}, \alpha_{1}, \ldots, \alpha_{p}, \beta_{1}, \beta_{2}, \ldots, \beta_{q}\right)^{\prime}$.

\subsubsection{Critério de Seleção de Modelos}

A seleção dos modelos ajustados por um $\operatorname{GARCH}(p, q)$ foi feita através do critério da Densidade Preditiva Ordenada descrita na seção 4.1. A Densidade Preditiva é definida como:

$$
\begin{gathered}
\pi\left(z_{T+k} \mid \mathbf{Z}\right)=\int_{\boldsymbol{\theta}} \pi\left(z_{T+k} \mid \mathbf{Z}, \boldsymbol{\theta}\right) \pi(\boldsymbol{\theta} \mid \mathbf{Z}) d \boldsymbol{\theta} \\
c_{k}=\pi\left(z_{T+k} \mid \mathbf{Z}\right)=\int_{\boldsymbol{\theta}}\left(\frac{1}{2 \pi h_{T+k}}\right)^{1 / 2} \exp \left\{-\frac{1}{2} \frac{z_{T+k}^{2}}{h_{T+k}}\right\} \pi(\boldsymbol{\theta} \mid \mathbf{Z}) d \boldsymbol{\theta}
\end{gathered}
$$

onde $\pi(\boldsymbol{\theta} \mid \mathbf{Z})$ é a densidade a posteriori para os parâmetros $\alpha_{0}, \alpha_{1}, \ldots, \alpha_{p}, \beta_{1}, \ldots, \beta_{q}, h_{T+k}$ é a volatilidade definida em (2.11) e a integral em (4.39) é uma integral múltipla no espaço dos parâmetros.

A densidade preditiva foi estimada através de Monte Carlo utilizando as observações $\alpha_{i}^{(l)}, i=0,1, \ldots, p$. e $\beta_{j}^{(l)}, j=1, \ldots, q$ obtidas por simulação MCMC considerando $h_{T+k}$ como uma função dos parâmetros $\boldsymbol{\theta}=\left(\alpha_{0}, \alpha_{1}, \ldots, \alpha_{p}, \beta_{1}, \beta_{2}, \ldots, \beta_{q}\right)^{\prime}$. A estimativa de Monte Carlo da densidade preditiva é dada por:

$$
\hat{c}_{n}=\frac{1}{M} \sum_{l=1}^{M} \frac{1}{\sqrt{2 \pi h_{T+k}\left(\theta^{(l)}\right)}} \exp \left\{-\frac{1}{2} \frac{z_{T+k}^{2}}{h_{T+k}\left(\theta^{(l)}\right)}\right\}
$$


O critério de seleção de modelo baseado na densidade preditiva ordenada consiste em escolher o modelo $l$ que apresenta o maior valor de $\hat{c}(l)=\prod_{n=T+1}^{T+K} \hat{c}_{n}(l)$.

\subsubsection{Previsão da Volatilidade}

A previsão da volatilidade $k$ passos à frente de um modelo $\operatorname{GARCH}(\mathrm{p}, \mathrm{q})$ é definida por:

$$
\hat{h}_{T+k}=E\left[h_{T+k} \mid \mathbf{Z}\right]
$$

Na abordagem bayesiana os parâmetros do modelo $\theta=\left(\alpha_{0}, \alpha_{1}, \ldots, \alpha_{p}, \beta_{1}, \beta_{2}, \ldots, \beta_{q}\right)^{\prime}$ são variáveis aleatórias, portanto:

$$
\begin{aligned}
E\left[h_{T+k} \mid \mathbf{Z}\right] & =E_{\boldsymbol{\theta}}\left[E\left[h_{T+k} \mid \mathbf{Z}, \boldsymbol{\theta}\right]\right] \\
\hat{h}_{T+k} & =E_{\boldsymbol{\theta}}\left[E\left[\alpha_{0}+\sum_{i=1}^{p} \alpha_{i} z_{T+k-i}^{2}+\sum_{j=1}^{q} \beta_{j} h_{T+k-j} \mid \mathbf{Z}, \boldsymbol{\theta}\right]\right] \\
\hat{h}_{T+k} & =E_{\boldsymbol{\theta}}\left[\alpha_{0}+\sum_{i=1}^{p} \alpha_{i} E\left[z_{T+k-i}^{2} \mid \mathbf{Z}\right]+\sum_{j=1}^{q} \beta_{j} E\left[h_{T+k-j} \mid \mathbf{Z}\right]\right.
\end{aligned}
$$

Para $k \leq(i, j)$ os valores $z_{T+k-i} \in \mathbf{Z}$ e as volatilidades $h_{T+k-j}$ são conhecidas com $h_{t}=\operatorname{var}\left(z_{t}\right) \approx \frac{1}{T} \sum_{t=1}^{T} z_{t}^{2}, t<m+1$, caso contrário temos:

$$
\begin{aligned}
E\left[z_{T+k-i}^{2} \mid \mathbf{Z}\right] & =E\left[h_{T+k-i} \varepsilon_{T+k-i}^{2} \mid \mathbf{Z}\right] \\
\hat{h}_{T+k-i} & =E\left[h_{T+k-i} \mid \mathbf{Z}\right]
\end{aligned}
$$

Assim, a previsão $k$ passos à frente na abordagem bayesiana pode ser calculada por (4.42) considerando

$$
E\left[z_{T+k-i}^{2} \mid \mathbf{Z}\right]= \begin{cases}z_{T+k-i}^{2} & \text { se } k \leq i \\ \hat{h}_{T+k-i} & \text { se } k>i\end{cases}
$$




$$
E\left[h_{T+k-j} \mid \mathbf{Z}\right]= \begin{cases}h_{T+k-j} & \text { se } k \leq j \\ \hat{h}_{T+k-j} & \text { se } k>j\end{cases}
$$

O valor esperado com relação a densidade a posteriori dos parâmetros $\boldsymbol{\theta}$ pode ser aproximado pela estimativa de Monte Carlo considerando a amostra $\boldsymbol{\theta}^{(l)}, l=1,2, \ldots, M$ gerada pelo algoritmo MCMC. Assim, temos:

$$
\hat{h}_{T+k}=\frac{1}{M} \sum_{l=1}^{M}\left(\alpha_{0}^{(l)}+\sum_{i=1}^{p} \alpha_{i}^{(l)} E\left[z_{T+k-i}^{2} \mid \mathbf{Z}\right]+\sum_{j=1}^{q} \beta_{j}^{(l)} E\left[h_{T+k-j} \mid \mathbf{Z}\right]\right)
$$

\subsubsection{Previsão do Preço no Modelo GARCH(p,q)}

\subsubsection{Previsão um passo à frente}

A previsão um passo a frente dos preços $\left\{y_{t}, t=T+1, \ldots, T+k\right\}$ é calculada por:

$$
\hat{y}_{T+k}=E_{\boldsymbol{\theta}}\left[E\left[y_{T+k} \mid \mathbf{Y}^{T+k-1}, \boldsymbol{\theta}\right]\right]
$$

onde $\mathbf{Y}^{T+k-1}=\left[y_{1}, \ldots, y_{T}, y_{T+1}, \ldots, y_{T+k-1}\right]$ e $\boldsymbol{\theta}=\left(\alpha_{0}, \alpha_{1}, \ldots, \alpha_{p}, \beta_{1}, \ldots, \beta_{q}\right)^{\prime}$ é o vetor de parâmetros do modelo.

Considerando a relação

$$
y_{T+k}=y_{T+k-1} e^{z_{T+k}}
$$

onde $z_{T+k} \sim N\left(0, h_{T+k}\right)$ e $h_{T+k}(\theta)=\alpha_{0}+\sum_{i=1}^{p} \alpha_{i} z_{T+k-i}^{2}+\sum_{j=1}^{q} \beta_{j} h_{T+k-j}$ sendo escrita como função não só das observações passadas e das volatilidades passadas (supostas conhecidas) mas também do vetor de parâmetros do modelo que são considerados como um vetor aleatório $(p+q+1)$ dimensional podemos calcular o valor esperado condicional em $(4,47)$ por: 


$$
E\left[y_{T+k-1} e^{z_{T+k}} \mid \mathbf{Y}^{(T+k-1)}, \theta\right]=y_{T+k-1} \exp \left(1 / 2 h_{T+k(\theta)}\right)
$$

Substituindo (4.49) em (4.47) temos:

$$
\hat{y}_{T+k}=E_{\theta}\left[y_{T+k-1} \exp \left(1 / 2 h_{T+k(\theta)}\right]\right.
$$

onde $y_{T+k-1}, y_{T+k-2, \cdots,} y_{1}$ são conhecidos no início do instante $T+k, k=1,2, \ldots, k$. Utilizando a amostra gerada pelo algoritmo de MCMC, $\boldsymbol{\theta}^{(l)}=\left(\alpha_{0}^{(l)}, \ldots, \alpha_{p}^{(l)}, \beta_{1}^{(l)}, \ldots, \beta_{q}^{(l)}\right), l=1,2, \ldots, M$ podemos avaliar o valor esperado em (4.50) por:

$$
\hat{y}_{T+k}=\frac{1}{M} \sum_{l=1}^{M} y_{T+k-1} \exp \left(1 / 2 h_{T+k\left(\theta^{(n)}\right)}\right)
$$

onde $h_{T+k}\left(\theta^{(l)}\right)=\alpha_{0}^{(l)}+\sum_{i=1}^{p} \alpha_{i}^{(l)} z_{T+k-i}^{2}+\sum_{j=1}^{q} \beta_{j}^{(l)} h_{T+k-j}$. A equação (4.51) pode ser usada para fazer a previsão um passo a frente para $k=1,2, \ldots, K$.

\subsubsection{Previsão $k$ passos à frente}

A previsão $k$ passos à frente de um modelo GARCH $(\mathrm{p}, q)$ é feita de maneira análoga ao dos modelos $\mathrm{ARCH}(\mathrm{p})$. Para calcular a previsão utilizamos as observações $\mathbf{Y}^{(T)}=\left(y_{1}, \ldots, y_{T}\right)^{\prime}$ e uma função $\phi\left(\mathbf{Y}^{*}, \mathbf{Y}^{(T)}\right)$ das observações $\mathbf{Y}^{(T)}$ e de $k$ valores futuros $\mathbf{Y}^{*}=\left(y_{T+1}, \ldots, y_{T+k}\right)^{\prime}$. A previsão destes valores futuros é calculada pelo seguinte valor esperado:

$$
E\left[\phi\left(\mathbf{Y}^{*}, \mathbf{Y}^{(T)}\right) \mid \mathbf{Y}^{(T)}\right]=E_{\boldsymbol{\theta}}\left[E\left[\phi\left(\mathbf{Y}^{*}, \mathbf{Y}^{(T)}\right) \mid \mathbf{Y}^{(T)}, \boldsymbol{\theta}\right]\right]
$$

Considerando o vetor aleatório $\theta$ de dimensão $(p+q+1)$ com densidade conjunta a posteriori $\pi\left(\theta \mid \mathbf{Y}^{(T)}\right)$ podemos calcular o valor esperado em (4.52) por: 


$$
E\left[\phi\left(\mathbf{Y}^{*}, \mathbf{Y}^{(T)}\right) \mid \mathbf{Y}^{(T)}\right]=\int_{\boldsymbol{\theta}} E\left[\phi\left(\mathbf{Y}^{*}, \mathbf{Y}^{(T)}\right) \mid \mathbf{Y}^{(T)}, \boldsymbol{\theta}\right] \pi\left(\boldsymbol{\theta} \mid \mathbf{Y}^{(T)}\right) d \boldsymbol{\theta}
$$

Através da amostra gerada pelo algoritmo MCMC para o vetor $\boldsymbol{\theta}^{(l)}=\left(\alpha_{0}^{(l)}, \ldots, \alpha_{p}^{(l)}, \beta_{1}^{(l)}, \ldots, \beta_{q}^{(l)}\right), l=1, \ldots, M$ podemos calcular $(4.53)$ por:

$$
E\left[\phi\left(\mathbf{Y}^{*}, \mathbf{Y}^{(T)}\right) \mid \mathbf{Y}^{(T)}\right]=\frac{1}{M} \sum_{l=1}^{M} E\left[\phi\left(\mathbf{Y}^{*}, \mathbf{Y}^{(T)}\right) \mid \mathbf{Y}^{(T)}, \boldsymbol{\theta}^{(l)}\right]
$$

$\mathrm{O}$ valor esperado em (4.54) pode ser avaliado gerando $\mathbf{y}^{*}$ das seguintes fórmulas recursivas:

(i) $h_{T+k}=\alpha_{0}+\sum_{i=1}^{p} \alpha_{i} z_{T+k-i}^{2}+\sum_{j=1}^{q} \beta_{j} h_{T+k-j}$

(ii) $z_{T+k} \sim N\left(0, h_{T+k}\right)$

(iii) $y_{T+k}=y_{T+k-1} e^{i_{T+k}}$

para $k=1,2, \ldots, K$. Dada a série gerada $y_{T+1}^{*}, \ldots, y_{T+k}^{*}, \phi\left(\mathbf{Y}^{*}, \mathbf{Y}^{(T)}\right)$ pode ser avaliada. Na implementação desse procedimento, $\theta^{(l)}$ é primeiro gerado da densidade conjunta a posteriori $\pi\left(\boldsymbol{\theta} \mid \mathbf{Y}^{(T)}\right)$. O vetor aleatório $\mathbf{Z}^{*}=\left(z_{T+1}, \ldots, z_{T+k}\right)^{\prime}$ é então construído e $\phi\left(\mathbf{Y}^{*}, \mathbf{Y}^{(T)}\right)$ é calculada. Esses procedimentos são repetidos $M$ vezes e a média de $\phi\left(\mathbf{Y}^{*}, \mathbf{Y}^{(T)}\right)$ pode então ser calculada.

\subsection{Avaliação da previsão um passo à frente}

Se a especificação do modelo estiver correta e os parâmetros estimados corretamente então $h_{t}$ é a esperança condicional de $z_{t}^{2}$. Portanto, a função perda erro médio quadrático $E\left\{\left(z_{t}^{2}-\theta_{t}\right)^{2} \mid z_{t-1}, z_{t-2}, \ldots\right\}$ pode ser minimizada com respeito a $\theta_{t}$ escolhendo $\theta_{t}=h_{t}$ para $h_{t}$ especificada em (2.3) e em (2.11). O valor médio de $\left(\hat{z}_{t}^{2}-\hat{h}_{t}\right)^{2}$ é dado por:

$$
E M Q=K^{-1} \sum_{t=T+1}^{T+K}\left(\hat{z}_{t}^{2}-\hat{h}_{t}\right)^{2}
$$


Outra função perda que pode ser utilizada é o erro médio absoluto EMA dada por:

$$
E M A=K^{-1} \sum_{t=T+1}^{T+K}\left|\hat{z}_{t}^{2}-\hat{h}_{t}\right|
$$

As funções perda $E M Q$ e $E M A$ foram utilizadas para comparar a volatilidade prevista pelos modelos $\mathrm{ARCH}(\mathrm{p})$. Outras funções perda podem ser utilizadas como pode ser visto em Hamilton e Susmel (1994).

As previsões para os preços foram avaliadas e comparadas através das estatísticas Erro Médio Quadrático $(E M Q)$, Erro Médio Absoluto $(E M A)$ e Erro Médio Absoluto Percentual $(E M A P)$. Estas estatísticas são calculadas pelas seguintes equações:

$$
\begin{aligned}
& E M Q=\frac{1}{k} \sum_{t=T+1}^{T+K}\left(y_{t}-\hat{y}_{t}\right)^{2} \\
& E M A=\frac{1}{K} \sum_{t=T+1}^{T+K}\left|y_{t}-\hat{y}_{t}\right| \\
& E M A P=\frac{1}{K} \sum_{t=T+1}^{T+K}\left|\frac{y_{t}-\hat{y}_{t} \mid}{y_{t}}\right|
\end{aligned}
$$

A análise desses erros para previsão mostra que baixos valores de $E M$ indicam menos vício na previsão enquanto as demais estatísticas são usadas como medida de precisão dos modelos. 


\section{Capítulo 5}

\section{Aplicações}

Neste capítulo apresentamos os resultados obtidos em duas séries geradas, $\mathrm{ARCH}(2)$, de tamanho 1000, e GARCH(1,1) de tamanho 500, e em três séries reais do mercado: telebrás, índice bovespa e cotação em dólar americano da moeda Iene Japonês. Todos os conjuntos de dados foram analisados via máxima verossimilhança e via MCMC utilizando o algoritmo Metrópolis-Hastings. Todas as análises foram feitas sob a suposição de normalidade.

\subsection{Resultados obtidos com o ajuste do modelo $\mathrm{ARCH}(\mathrm{p})$}

\subsubsection{Série Gerada}

Primeiramente selecionamos o "melhor modelo" segundo os critérios AIC e BIC obtidos no ajuste dos modelos $\mathrm{ARCH}(1), \mathrm{ARCH}(2)$ e $\mathrm{ARCH}(3)$. Na abordagem bayesiana utilizamos o critério da densidade preditiva ordenada. Esta análise foi feita com a finalidade de validar os algoritmos desenvolvidos pois como a série foi gerada por um $\operatorname{ARCH}(2)$ conhecido é de se esperar que o modelo escolhido seja o mais próximo do modelo verdadeiro.

A figura 1 apresenta os retornos do logaritmo da série. Podemos observar a alta volatilidade da série gerada pelo modelo $\mathrm{ARCH}(2)$.

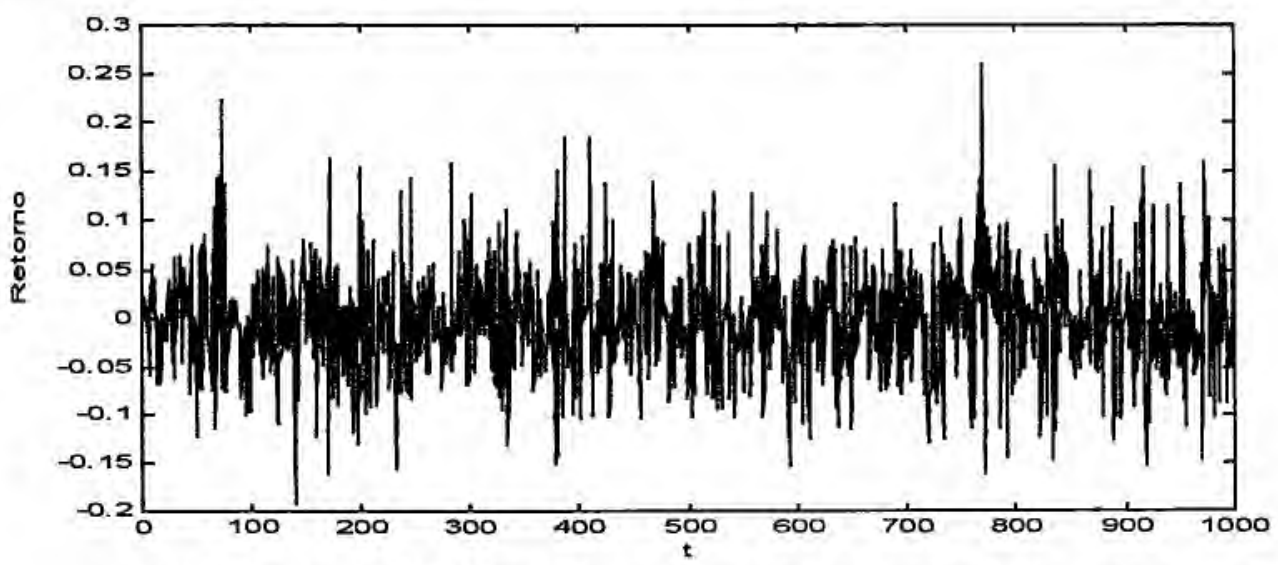

Figura 1: $\operatorname{ARCH}(2) \operatorname{com} \alpha_{0}=0.002, \alpha_{1}=0.2$ e $\alpha_{2}=0.3$ 

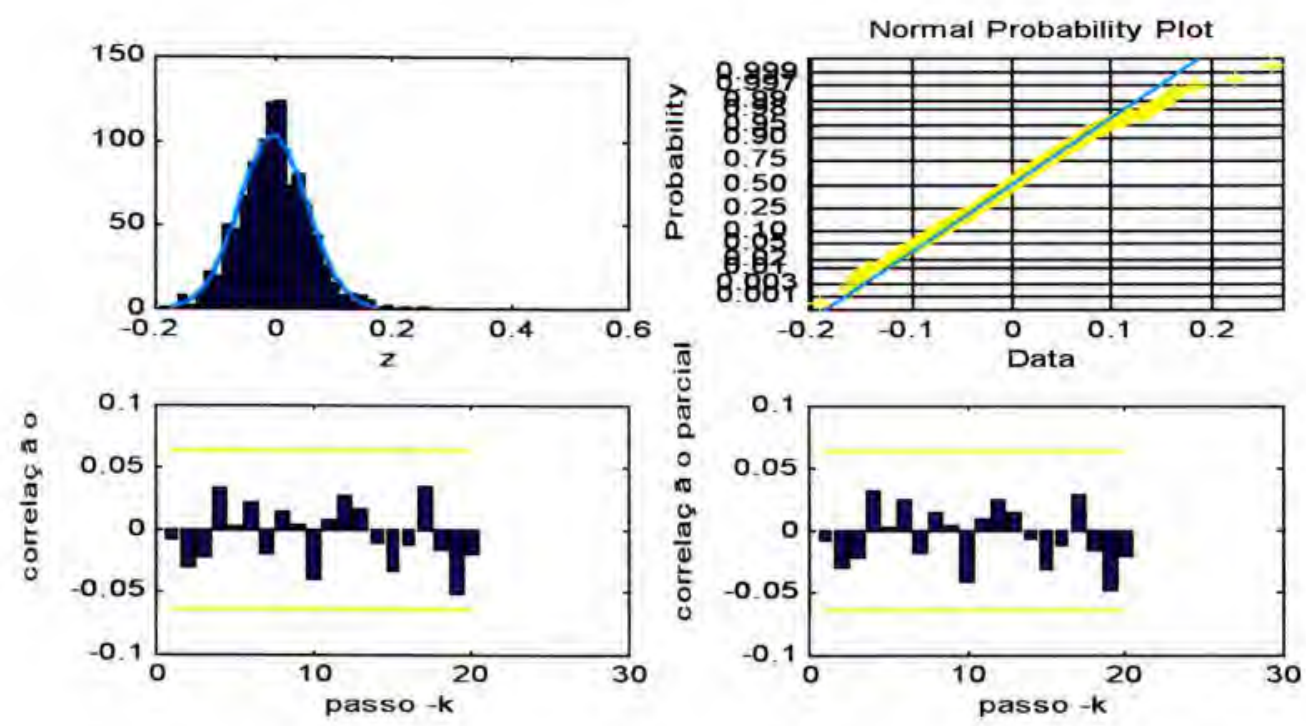

Figura 2: Histograma, gráficos normal probabilístico, auto-correlação e correlação parcial da série $z_{t}$ gerada

Pelos gráficos acima observamos que a série apresenta distribuição normal e os gráficos da correlação e da correlação parcial indicam que a série $z_{t}$ não apresenta correlação.

Tabela 1: Critérios de seleção de modelos

\begin{tabular}{lccc}
\hline \multicolumn{4}{c}{ ARCH } \\
\hline Ordem do Modelo & $\mathbf{1}$ & $\mathbf{2}$ & $\mathbf{3}$ \\
Log Verossimilhança & $1.39 \mathrm{e}+003$ & $1.42 \mathrm{e}+003$ & - \\
AIC & -2.8710 & -2.9358 & - \\
BIC & -2.8609 & -2.9208 & - \\
CPO & $3.70 \mathrm{e}+015$ & $6.10 \mathrm{e}+015$ & $5.73 \mathrm{e}+015$ \\
\hline
\end{tabular}

Podemos observar que o modelo $\mathrm{ARCH}(2)$ é o "melhor modelo" pois apresenta o menor valor tanto no critério AIC quanto no BIC. Não houve convergência dos parâmetros no ajuste de um $\mathrm{ARCH}$ de ordem 3 na estimativa via máxima verossimilhança. Pelo critério da densidade preditiva ordenada o modelo escolhido é o que tem o maior valor da $\mathrm{CPO}$, ou seja, o modelo 2 .

Após a escolha do "melhor modelo" apresentamos as estimativas dos parâmetros do modelo escolhido via máxima verossimilhança e MCMC utilizando o algoritmo MetrópolisHastings. Os resultados estão apresentados na tabela 2. 
Tabela 2: Estimativas dos parâmetros do modelo ARCH(2)

\begin{tabular}{lccccccc}
\hline & \multicolumn{3}{c}{ M.V } & \multicolumn{5}{c}{ Bayesiano } \\
\cline { 2 - 7 } & $\hat{\boldsymbol{\alpha}}$ & $\begin{array}{c}\text { Intervalo de } \\
\text { Confiança }\end{array}$ & Média & Moda & Mediana & $\begin{array}{c}\text { Desvio } \\
\text { Padrão }\end{array}$ & $\begin{array}{c}\text { Intervalo de } \\
\text { Credibilidade }\end{array}$ \\
\hline$\alpha_{0}$ & 0.0020 & $(0.0016 ; 0.0023)$ & 0.0020 & 0.0019 & 0.0020 & 0.0002 & $(0.0016 ; 0.0023)$ \\
$\alpha_{1}$ & 0.1801 & $(0.0913 ; 0.2689)$ & 0.1914 & 0.1765 & 0.1891 & 0.0470 & $(0.1040 ; 0.2849)$ \\
$\alpha_{2}$ & 0.2485 & $(0.1496 ; 0.3474)$ & 0.2556 & 0.2719 & 0.2529 & 0.0463 & $(0.1732 ; 0.3468)$ \\
\hline
\end{tabular}

As estimativas dos parâmetros obtidas pelos dois métodos apresentaram-se muito próximas e o intervalo de credibilidade para $\alpha_{2}$ mostrou-se mais preciso na abordagem bayesiana.

Na implementação do algoritmo Metrópolis-Hastings, para cada parâmetro, foram simuladas cinco cadeias com 3.000 iterações cada, descartadas $50 \%$ de cada uma e tomados valores espaçados de 15 em 15 totalizando uma amostra de tamanho 500 .

A convergência dos parâmetros foi verificada pelo critério de Gelman e Rubin (1992) obtendo-se $\sqrt{\hat{R}}<1.2$ para todos os parâmetros.

Nos gráficos da figura 3 apresentamos os histogramas construídos com as amostras selecionadas para os parâmetros $\alpha_{0}, \alpha_{1}$ e $\alpha_{2}$, respectivamente.
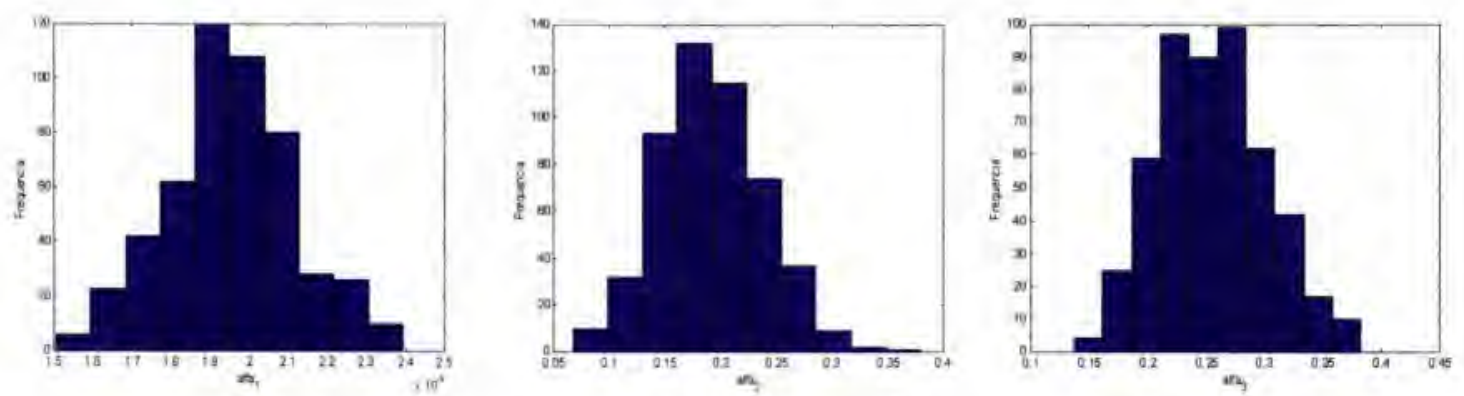

Figura 3: Distribuição a Posteriori Marginal: $\alpha_{0}, \alpha_{1}$ e $\alpha_{2}$
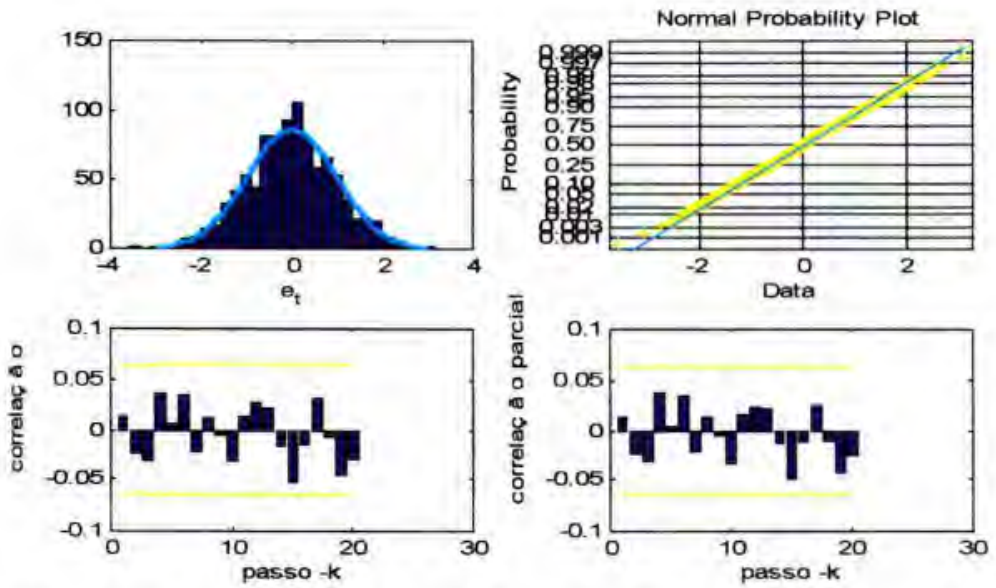

Figura 4: Histograma, gráficos normal probabilístico, auto-correlação e correlação parcial dos resíduos - série gerada 
Os gráficos da figura 4 resumem a análise dos resíduos para o modelo selecionado na abordagem bayesiana. Observamos que os resíduos estão normalmente distribuídos e não apresentam correlação significativa, portanto, o modelo escolhido mostra-se adequado.

A figura 5 apresenta o gráfico da volatilidade estimada para um período de 970 dias. Os últimos 30 dias foram deixados fora do ajuste para serem utilizados no cálculo da previsão da volatilidade e do preço.

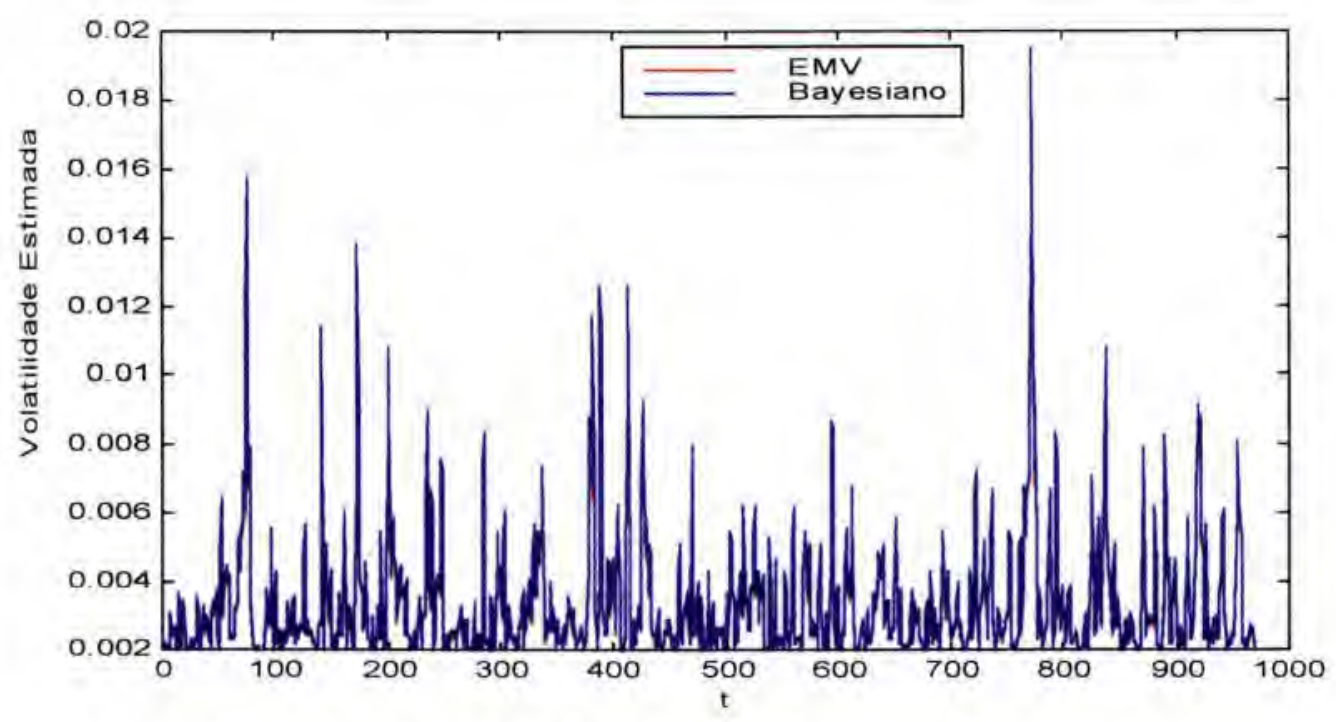

Figura 5: Gráfico da volatilidade estimada - série gerada

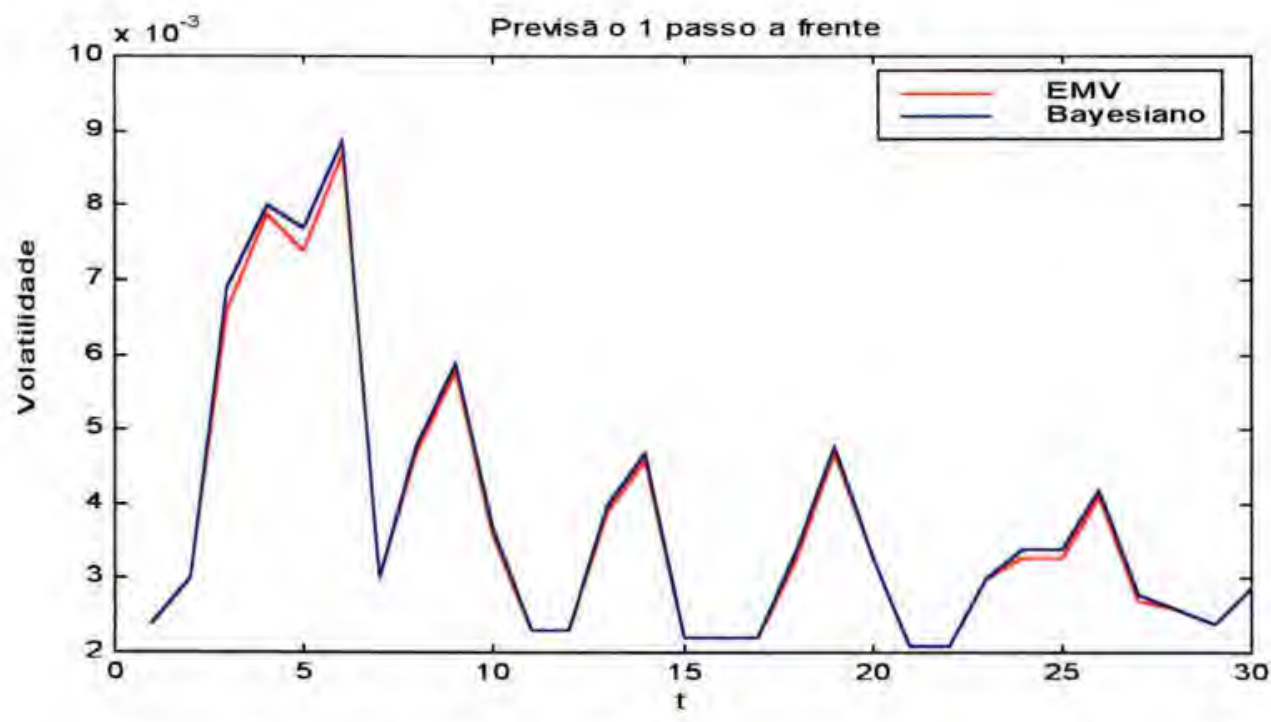

Figura 6: Previsão da volatilidade 1 passo a frente - série gerada 


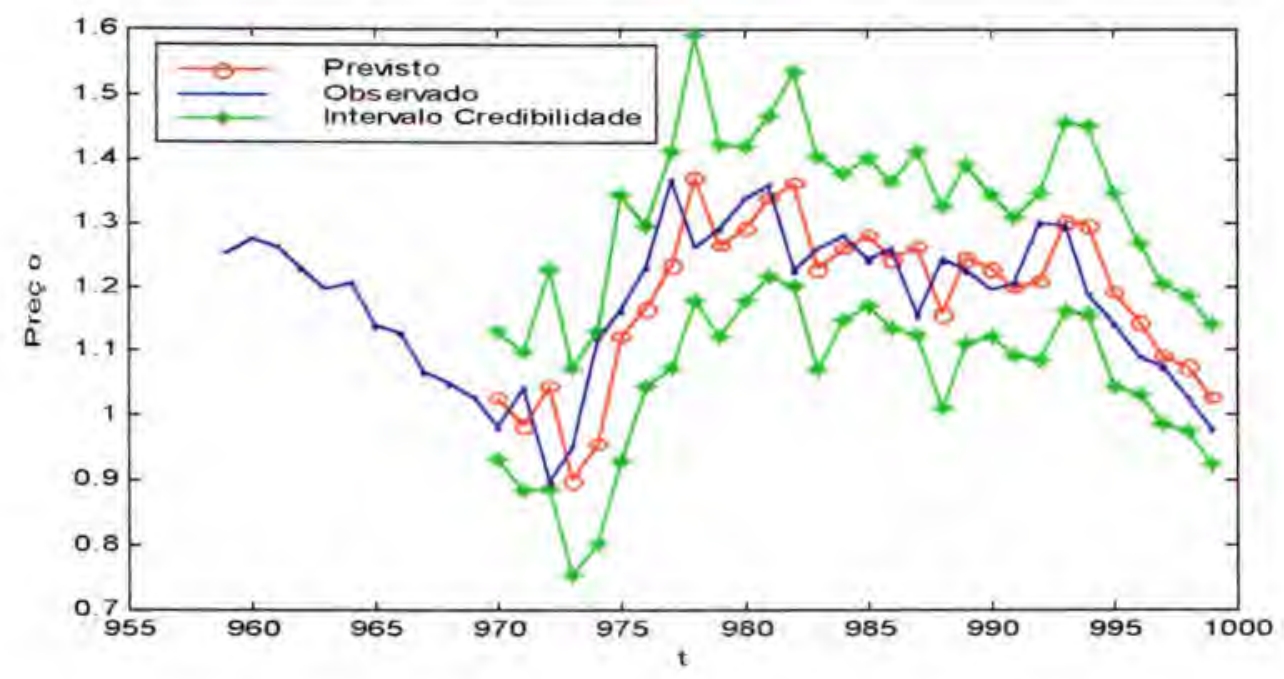

Figura 7: Previsão do preço 1 passo a frente - série gerada

As figuras 6 e 7 apresentam a previsão um passo à frente da volatilidade e do preço, respectivamente, durante um período de 30 dias. O gráfico da figura 7 foi construído através dos resultados obtidos pela abordagem bayesiana que é similar aos obtidos via máxima verossimilhança

\subsubsection{Série Índice Bovespa}

Esta série consiste de 651 observações diárias do índice de fechamento da bolsa de valores de São Paulo no período de 02/01/96 a 01/02/99.

A figura 8 apresenta os retornos da série calculados pela equação $z_{t}=\ln \left(\frac{y_{t}}{y_{t-1}}\right)$ onde $y_{t}$ é o preço do ativo no instante $t$

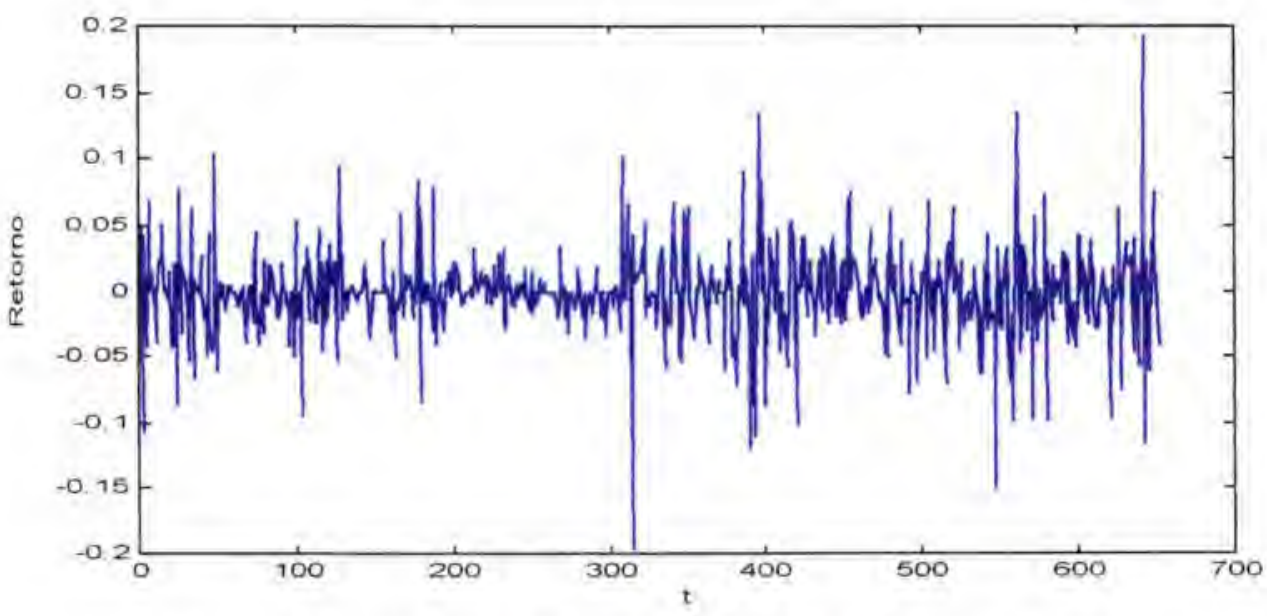

Figura 8: Retornos do logaritmo da série ibovespa 

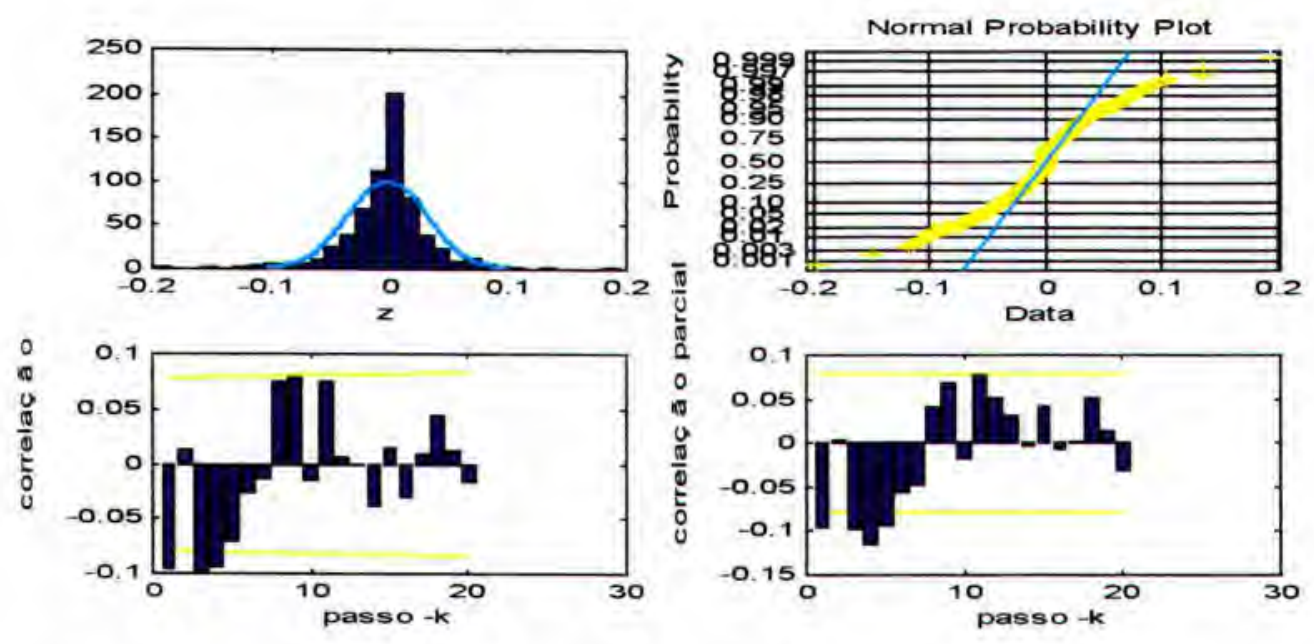

Figura 9: Histograma, gráficos normal probabilístico, auto-correlação e correlação parcial da série $z_{t}$ - ibovespa

O gráfico normal probabilístico indica que a distribuição real da série $z_{t}$ não é normal. No entanto, neste trabalho, vamos adotar uma aproximação normal para a distribuição de $z_{t}$. Além disso, o gráfico da função de auto correlação indica a presença de correlação entre os valores de $z_{t}$. Estas correlações certamente podem contribuir para a imprecisão dos ajustes dos modelos ARCH para esta série. No entanto, visto que estas correlações são pequenas $(<0.15)$, consideramos $z_{t}$ como um processo não correlacionado.

Tabela 3: Critérios de seleção de modelos

\begin{tabular}{lccc}
\hline \multicolumn{4}{c}{ ARCH } \\
\hline Ordem do Modelo & $\mathbf{1}$ & $\mathbf{2}$ & $\mathbf{3}$ \\
Log Verossimilhança & $1.2560 \mathrm{e}+003$ & $1.2583 \mathrm{e}+003$ & $1.2768 \mathrm{e}+003$ \\
AIC & -4.0387 & -4.0429 & -4.0990 \\
BIC & -4.0244 & -4.0215 & -4.0705 \\
CPO & $5.21 \mathrm{e}+013$ & $3.15 \mathrm{e}+012$ & $2.74 \mathrm{e}+017$ \\
\hline
\end{tabular}

Pela tabela 3 observamos que o modelo escolhido pelos critérios AIC/BIC e CPO foi o modelo $\mathrm{ARCH}(3)$.

Tabela 4: Estimativas dos parâmetros do modelo ARCH(3)

\begin{tabular}{cccccccc}
\hline & \multicolumn{3}{c}{ M.V } & \multicolumn{4}{c}{ Bayesiano } \\
\cline { 2 - 7 } & $\hat{\boldsymbol{a}}$ & $\begin{array}{c}\text { Intervalo de } \\
\text { Confiança }\end{array}$ & Média & Moda & Mediana & $\begin{array}{c}\text { Desvio } \\
\text { Padrão }\end{array}$ & $\begin{array}{c}\text { Intervalo de } \\
\text { Credibilidade }\end{array}$ \\
\hline$\alpha_{0}$ & 0.0006 & $(0.0005 ; 0.0007)$ & 0.0005 & 0.0006 & 0.0005 & 0.0001 & $(0.0004 ; 0.0007)$ \\
$\alpha_{1}$ & 0.2151 & $(0.0997 ; 0.3304)$ & 0.2497 & 0.2204 & 0.2410 & 0.0767 & $(0.1221 ; 0.4256)$ \\
$\alpha_{2}$ & 0.0626 & $(-0.0100 ; 0.1352)$ & 0.1055 & 0.1010 & 0.1009 & 0.0373 & $(0.0441 ; 0.1910)$ \\
$\alpha_{3}$ & 0.2381 & $(0.1175 ; 0.3587)$ & 0.2624 & 0.2456 & 0.2587 & 0.0662 & $(0.1471 ; 0.4041)$ \\
\hline
\end{tabular}


As estimativas de máxima verossimilhança e bayesiana apresentaram-se próximas e o intervalo de confiança mostrou-se mais preciso, exceto para o parâmetro $\alpha_{2}$ que apresentou intervalo de confiança com limite inferior negativo.

$\mathrm{Na}$ implementação do algoritmo $\mathrm{M}-\mathrm{H}$ utilizamos o mesmo procedimento usado no caso da série gerada. O critério de Gelman e Rubin foi verificado obtendo-se $\sqrt{\hat{R}}<1.2$ para todos os parâmetros.

Os histogramas construídos com as amostras selecionadas para os parâmetros $\alpha_{0}, \alpha_{1}, \alpha_{2}$ e $\alpha_{3}$ estão na figura 10 .
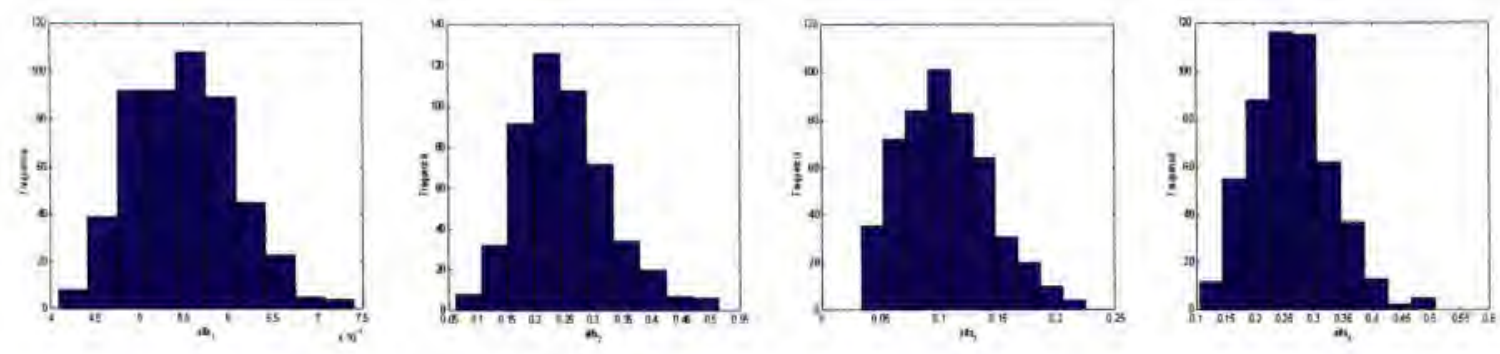

Figura 10: Distribuição a Posteriori Marginal: $\alpha_{0}, \alpha_{1}, \alpha_{2}$ e $\alpha_{3}$
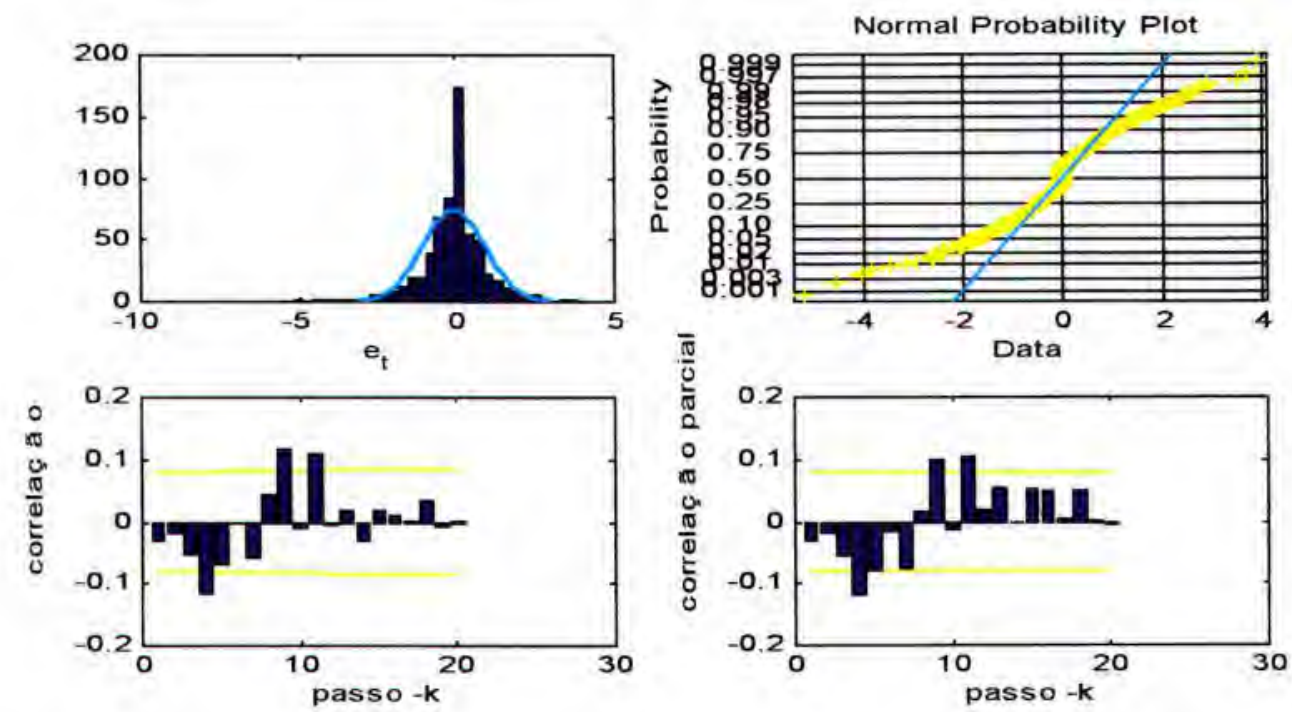

Figura 11: Histograma, gráficos normal probabilístico, auto-correlação e correlação parcial dos resíduos - série ibovespa

Os gráficos da figura 11 apresentam a análise dos resíduos para o modelo $\mathrm{ARCH}(3)$. Podemos verificar que a distribuição dos resíduos se distancia da distribuição normal e apresenta uma pequena correlação. O modelo escolhido serve como uma aproximação que certamente poderá ser melhorada com outras hipóteses sobre a distribuição de $z_{t}$. 


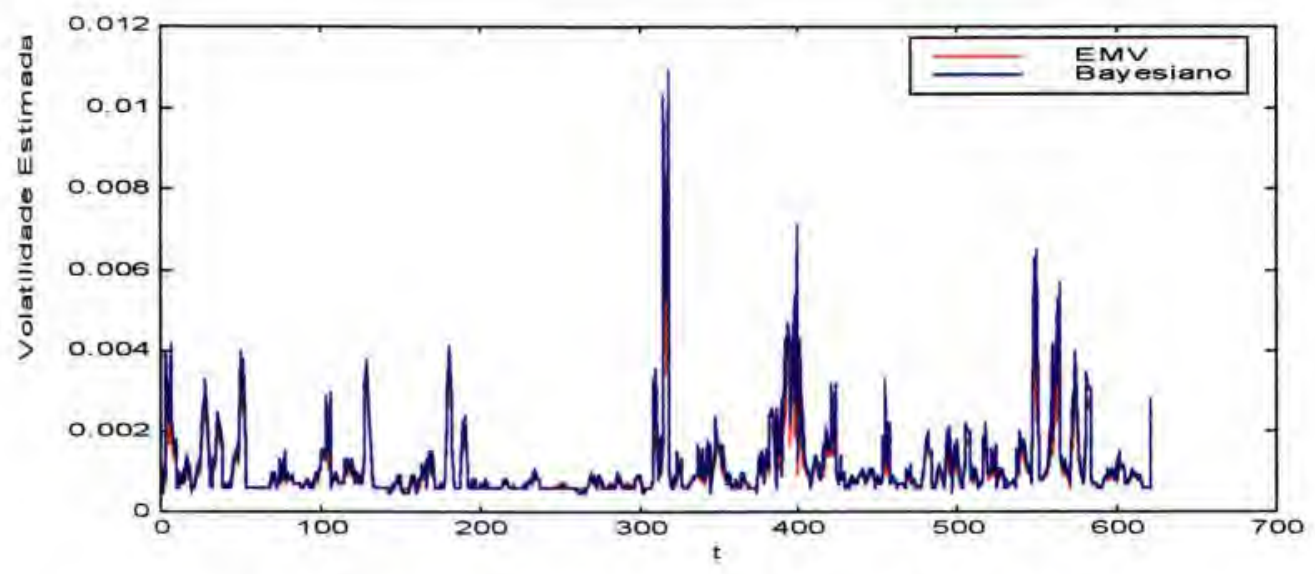

Figura 12: Gráfico da volatilidade estimada - série ibovespa

A figura 12 apresenta o gráfico da volatilidade estimada para um período de 621 dias. Os 30 dias restantes foram deixados fora do ajuste para serem utilizados no cálculo da previsão da volatilidade e do preço.

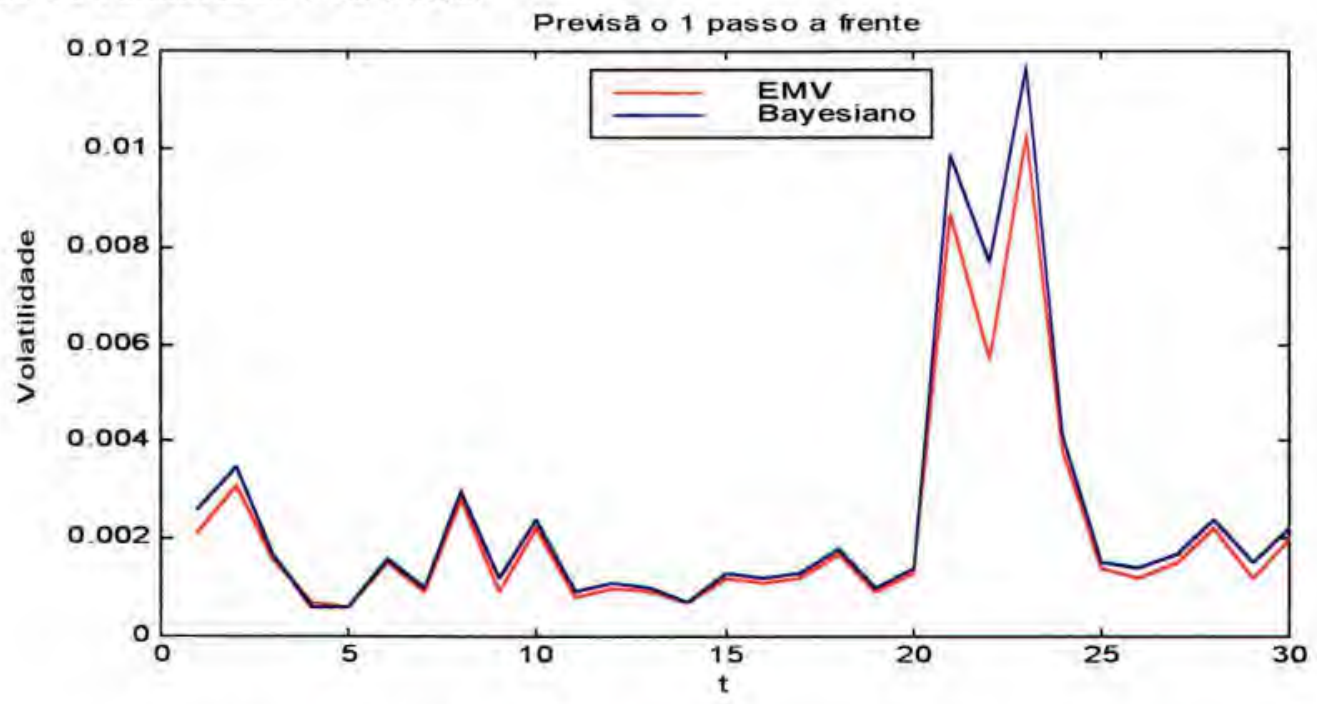

Figura 13: Previsão da volatilidade 1 passo a frente - série ibovespa

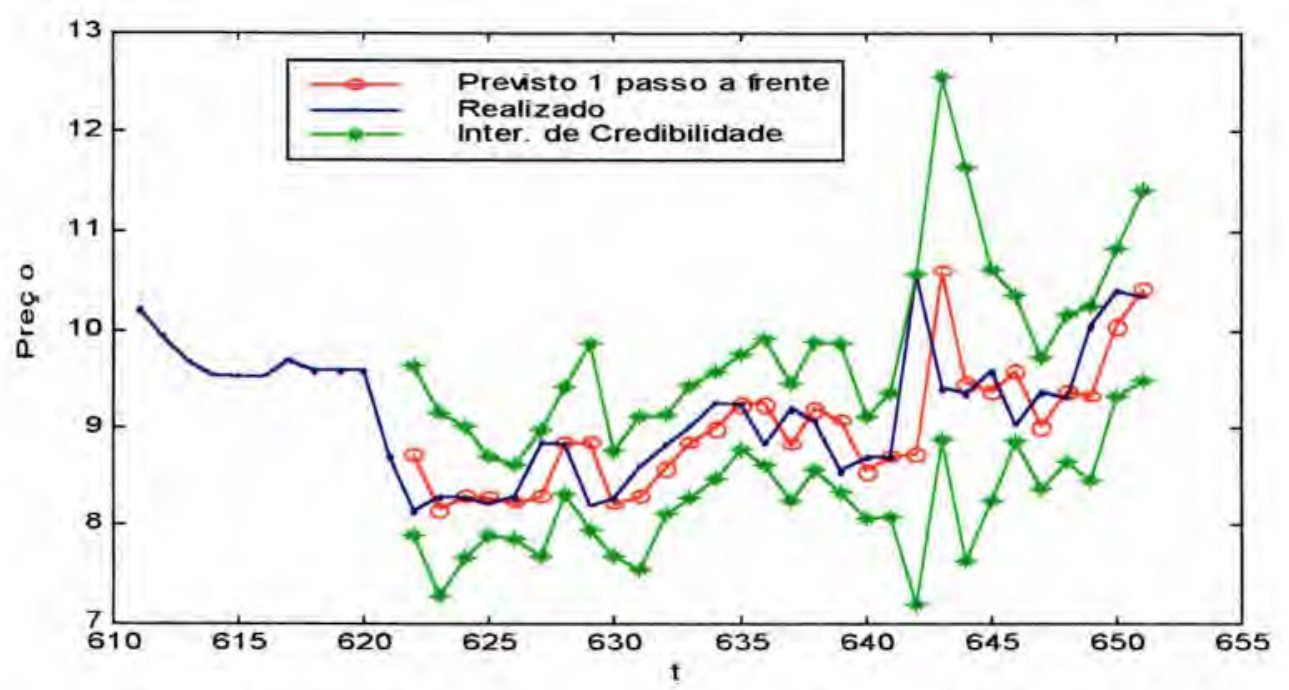

Figura 14: Previsão do preço 1 passo a frente - série ibovespa 
As figuras 13 e 14 apresentam a previsão um passo à frente da volatilidade e do preço, respectivamente, durante um periodo de 30 dias. O gráfico da figura 14 foi construído através dos resultados obtidos pela abordagem bayesiana que é similar aos obtidos via máxima verossimilhança. Notamos que todas observações futuras ocorreram dentro do intervalo previsto com $95 \%$ de credibilidade.

\subsubsection{Série Telebrás}

A série de ações da Telebrás é composta de 986 valores amostrados diariamente durante o período de $01 / 08 / 94$ a 13/03/97. Tal série representa o valor de fechamento das ações da Telebrás.

A figura 15 mostra a variação na volatilidade da série sugerindo o uso de modelos com heterocedasticidade.

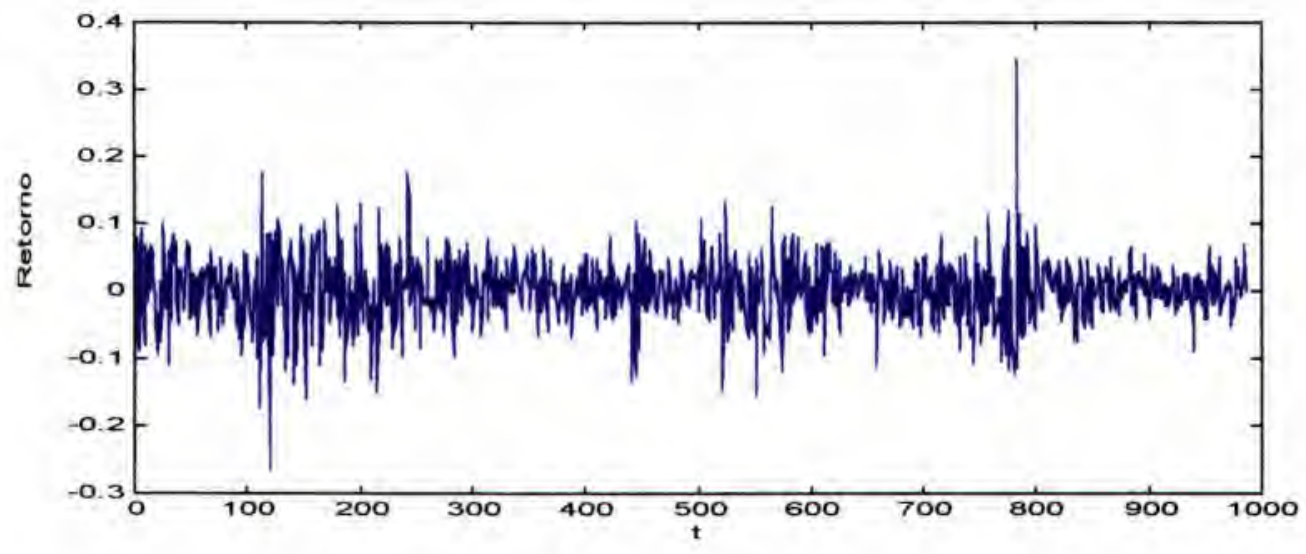

Figura 15: Retornos do logaritmo da série telebrás
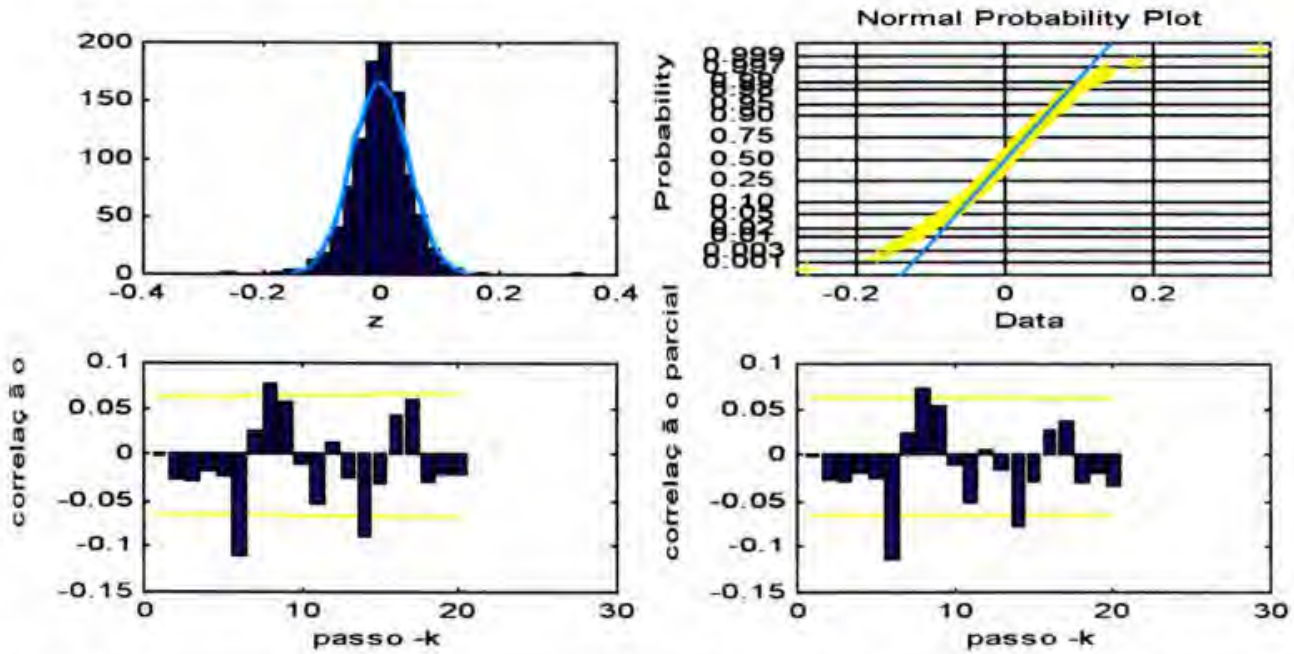

Figura 16: Histograma, gráficos normal probabilístico, auto-correlação e correlação parcial da série $z_{t}$ - série telebrás 
Conforme vimos no caso da série de índice bovespa, a série telebrás não apresenta distribuição normal como pode ser visto pelo gráfico normal probabilístico. Porém, vamos adotar a distribuição normal para $z_{t}$. Também podemos verificar uma pequena correlação entre os valores de $z_{t}$.

Tabela 5: Critérios de seleção de modelos

\begin{tabular}{lccc}
\hline \multicolumn{4}{c}{ ARCH } \\
\hline Ordem do Modelo & $\mathbf{5}$ & $\mathbf{6}$ & $\mathbf{7}$ \\
Log Verossimilhança & $1.6055 \mathrm{e}+003$ & $1.6176 \mathrm{e}+003$ & $1.6208 \mathrm{e}+003$ \\
AIC & -3.3463 & -3.3694 & -3.3742 \\
BIC & -3.3158 & -3.3338 & -3.3335 \\
CPO & $6.034 \mathrm{e}+026$ & $7.442 \mathrm{e}+026$ & $9.712 \mathrm{e}+026$ \\
\hline
\end{tabular}

Pela tabela 5 observamos que o modelo escolhido pelos critérios AIC e CPO foi o modelo $\operatorname{ARCH}(7)$.

Tabela 6: Estimativas dos parâmetros do modelo ARCH(7)

\begin{tabular}{lccccccc}
\hline & \multicolumn{3}{c}{ M.V } & \multicolumn{5}{c}{ Bayesiano } \\
\cline { 2 - 7 } & $\hat{\boldsymbol{\alpha}}$ & $\begin{array}{c}\text { Intervalo de } \\
\text { Confiança }\end{array}$ & Média & Moda & Mediana & $\begin{array}{c}\text { Desvio } \\
\text { Padrão }\end{array}$ & $\begin{array}{c}\text { Intervalo de } \\
\text { Credibilidade }\end{array}$ \\
\hline$\alpha_{0}$ & 0.0006 & $(0.0004 ; 0.0008)$ & 0.0006 & 0.0005 & 0.0006 & 0.0001 & $(0.0004 ; 0.0008)$ \\
$\alpha_{1}$ & 0.1068 & $(0.0257 ; 0.1880)$ & 0.1222 & 0.1276 & 0.1219 & 0.0397 & $(0.0556 ; 0.2057)$ \\
$\alpha_{2}$ & 0.1482 & $(0.0592 ; 0.2373)$ & 0.1526 & 0.1238 & 0.1474 & 0.0432 & $(0.0782 ; 0.2453)$ \\
$\alpha_{3}$ & 0.1096 & $(0.0270 ; 0.1923)$ & 0.1318 & 0.1277 & 0.1270 & 0.0400 & $(0.0650 ; 0.2179)$ \\
$\alpha_{4}$ & 0.0935 & $(0.0136 ; 0.1735)$ & 0.1222 & 0.1046 & 0.1164 & 0.0398 & $(0.0522 ; 0.2102)$ \\
$\alpha_{5}$ & 0.0569 & $(-0.0150 ; 0.1289)$ & 0.0865 & 0.0814 & 0.0838 & 0.0333 & $(0.0304 ; 0.1612)$ \\
$\alpha_{6}$ & 0.1079 & $(0.0268 ; 0.1890)$ & 0.1147 & 0.1009 & 0.1107 & 0.0385 & $(0.0480 ; 0.2013)$ \\
$\alpha_{7}$ & 0.1091 & $(0.0278 ; 0.1905)$ & 0.1312 & 0.1135 & 0.1292 & 0.0422 & $(0.0609 ; 0.2241)$ \\
\hline
\end{tabular}

Pela tabela 6 podemos observar que as estimativas via máxima verossimilhança e bayesianas, para alguns valores, não estão próximas e o intervalo de confiança para $\alpha_{5}$ apresentou valor negativo no limite inferior.

$\mathrm{Na}$ implementação do algoritmo Metrópolis-Hastings, para cada parâmetro, foram simuladas cinco cadeias com 6.000 iterações cada, descartadas $50 \%$ de cada uma e tomados valores espaçados de 15 em 15 totalizando uma amostra de tamanho 1000.

O critério de Gelman e Rubin foi verificado, obtendo-se $\sqrt{\hat{R}}<1.2$ para todos os parâmetros.

A figura 17 apresenta os histogramas construídos com as amostras selecionadas para os parâmetros do modelo selecionado. 

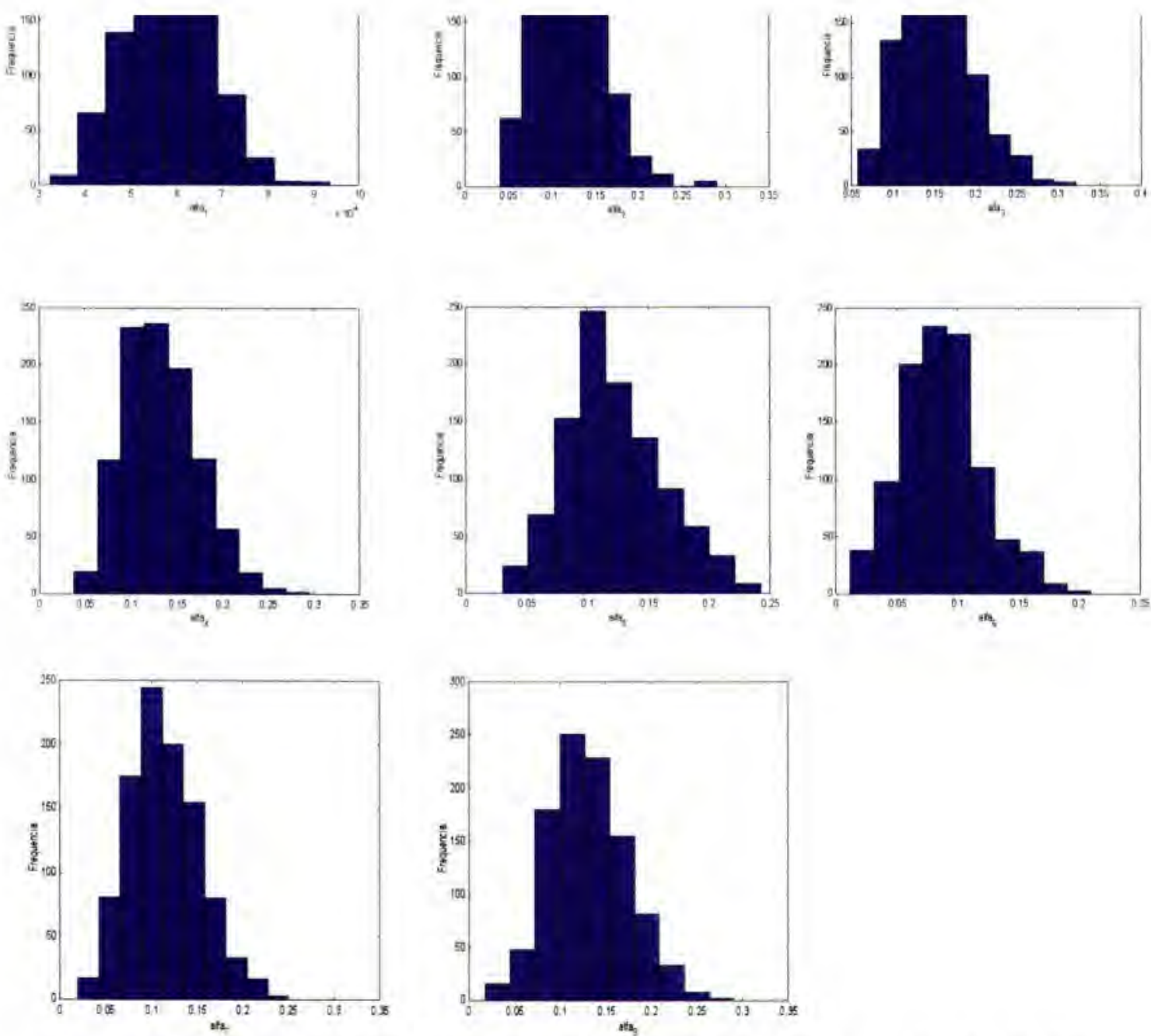

Figura 17: Distribuição a Posteriori Marginal: $\alpha_{0}, \alpha_{1}, \alpha_{2}, \alpha_{3}, \alpha_{4}, \alpha_{5}, \alpha_{6}$ e $\alpha_{7}$
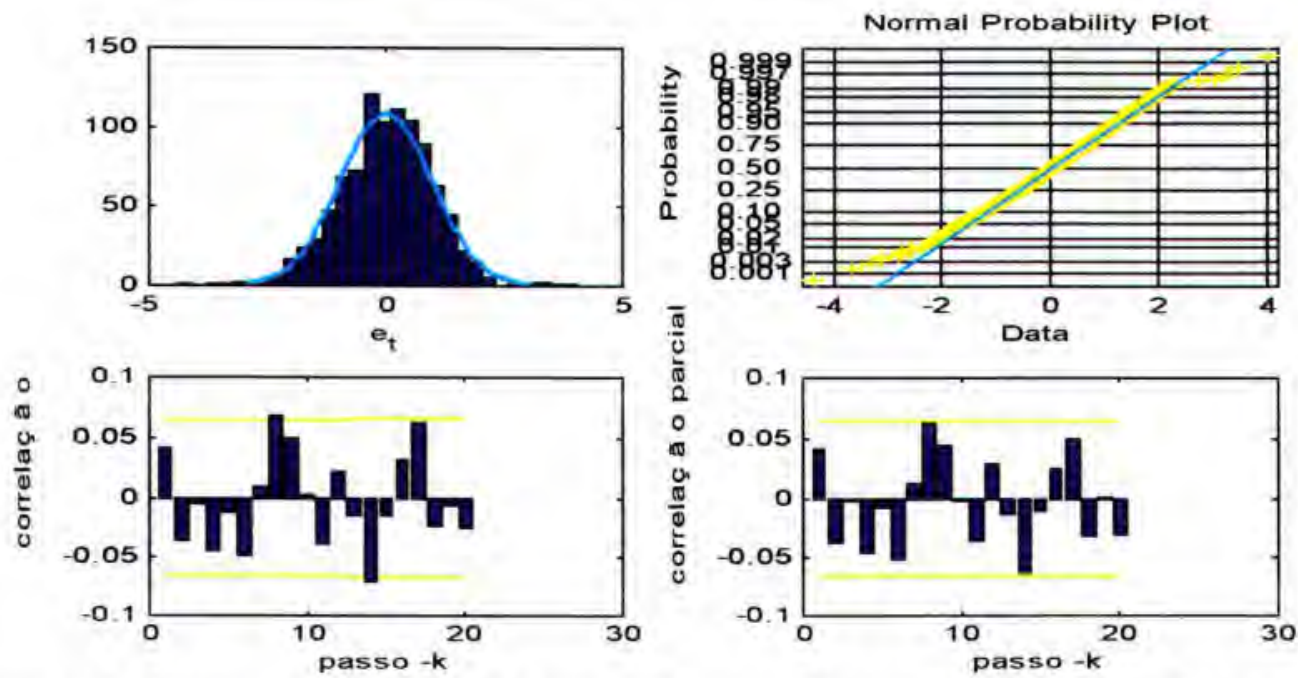

Figura 18: Histograma, gráficos normal probabilístico, auto-correlação e correlação parcial dos resíduos - série telebrás 
Através da análise gráfica feita pela figura 18 observamos que os resíduos se distanciam da normalidade nos extremos e praticamente não apresentam correlação.

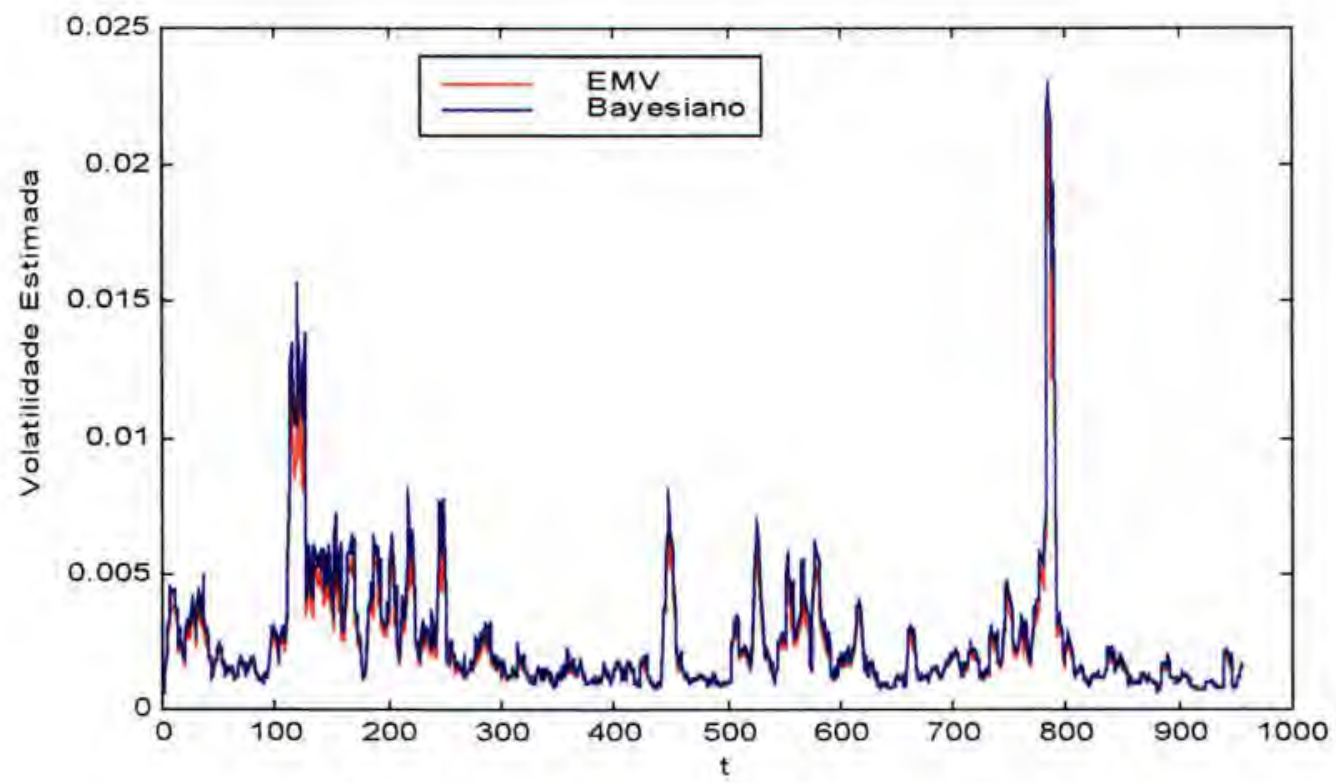

Figura 19: Gráfico da volatilidade estimada-série telebrás

Como nas séries anteriormente analisadas, a figura 19 apresenta o gráfico da volatilidade estimada para um período de 956 dias sendo o restante utilizado no cálculo da previsão da volatilidade e do preço.

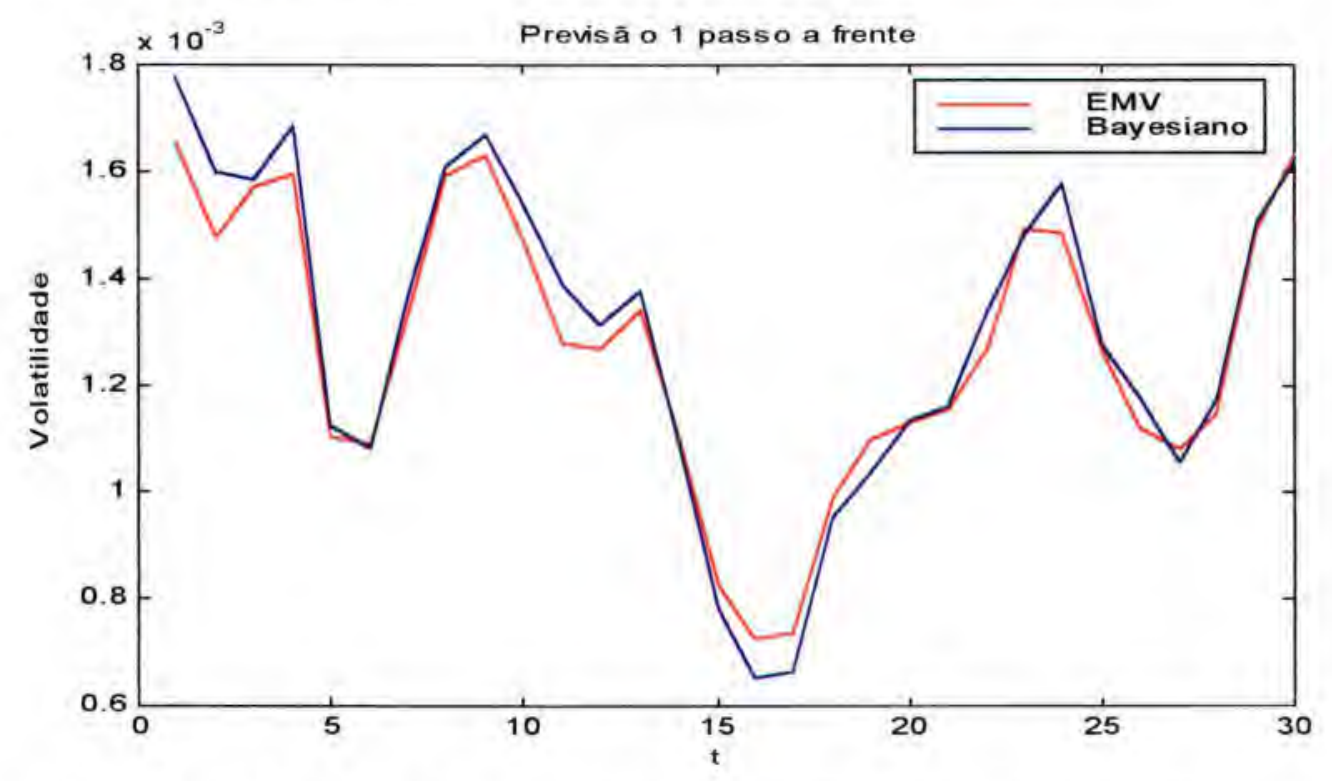

Figura 20: Previsão da volatilidade 1 passo a frente - série telebrás 


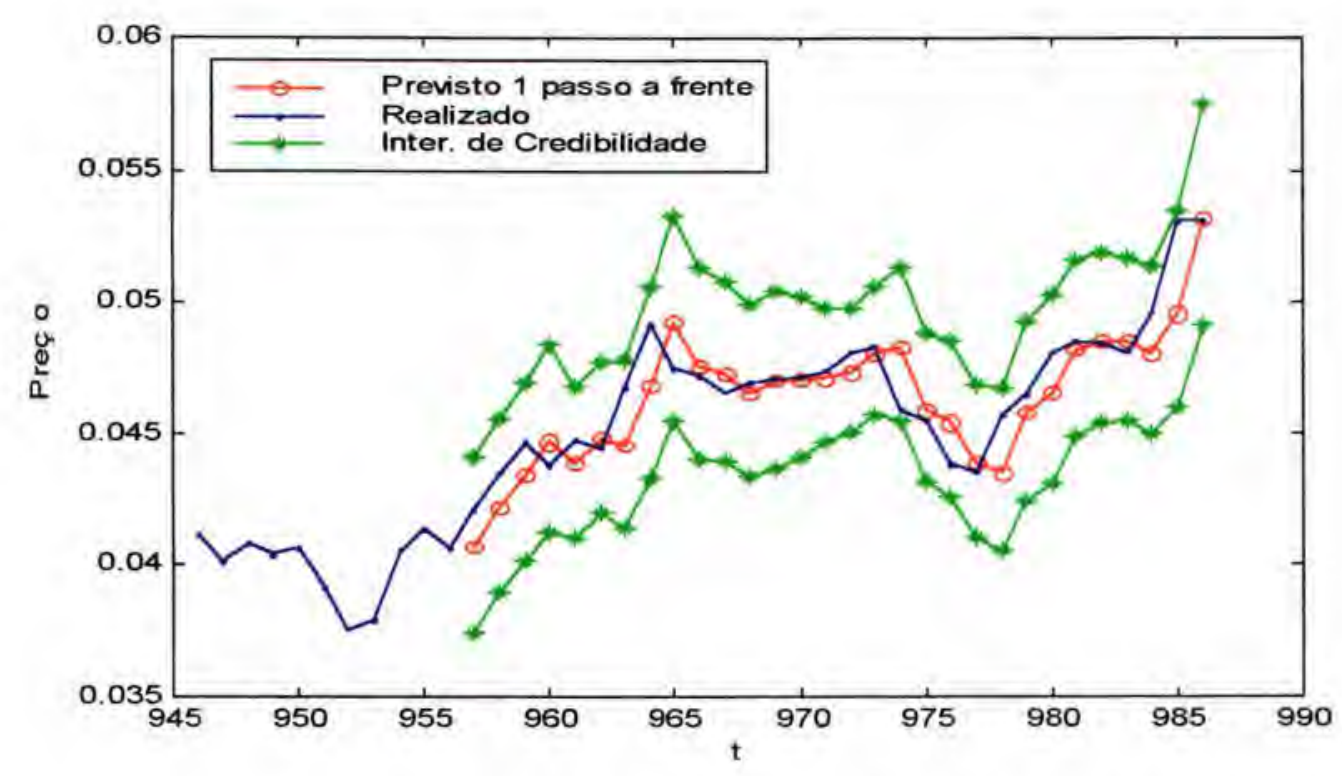

Figura 21: Previsão do preço 1 passo a frente - série telebrás

As figuras 20 e 21 apresentam a previsão um passo à frente da volatilidade e do preço, respectivamente, durante um período de 30 dias. A previsão do preço apresentada foi feita com os resultados obtidos na abordagem bayesiana.

\subsubsection{Série Cotação em Dólar Americano da moeda lene Japonês}

A série cotação em Dólar Americano da moeda Iene Japonês consiste de 3510 valores amostrados diariamente entre o período de $01 / 06 / 73$ a $21 / 05 / 87$. A figura 22 apresenta o retorno do logaritmo da série.

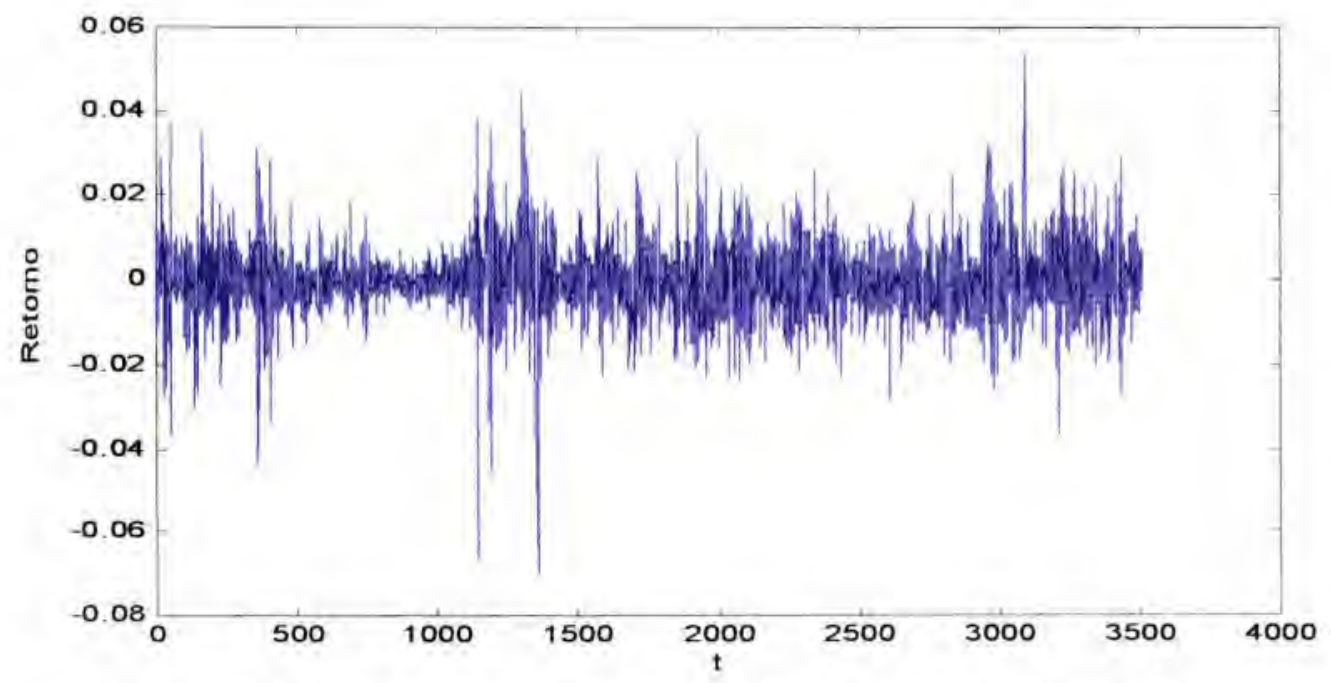

Figura 22: Retornos do logaritmo da série moeda Iene Japonês 

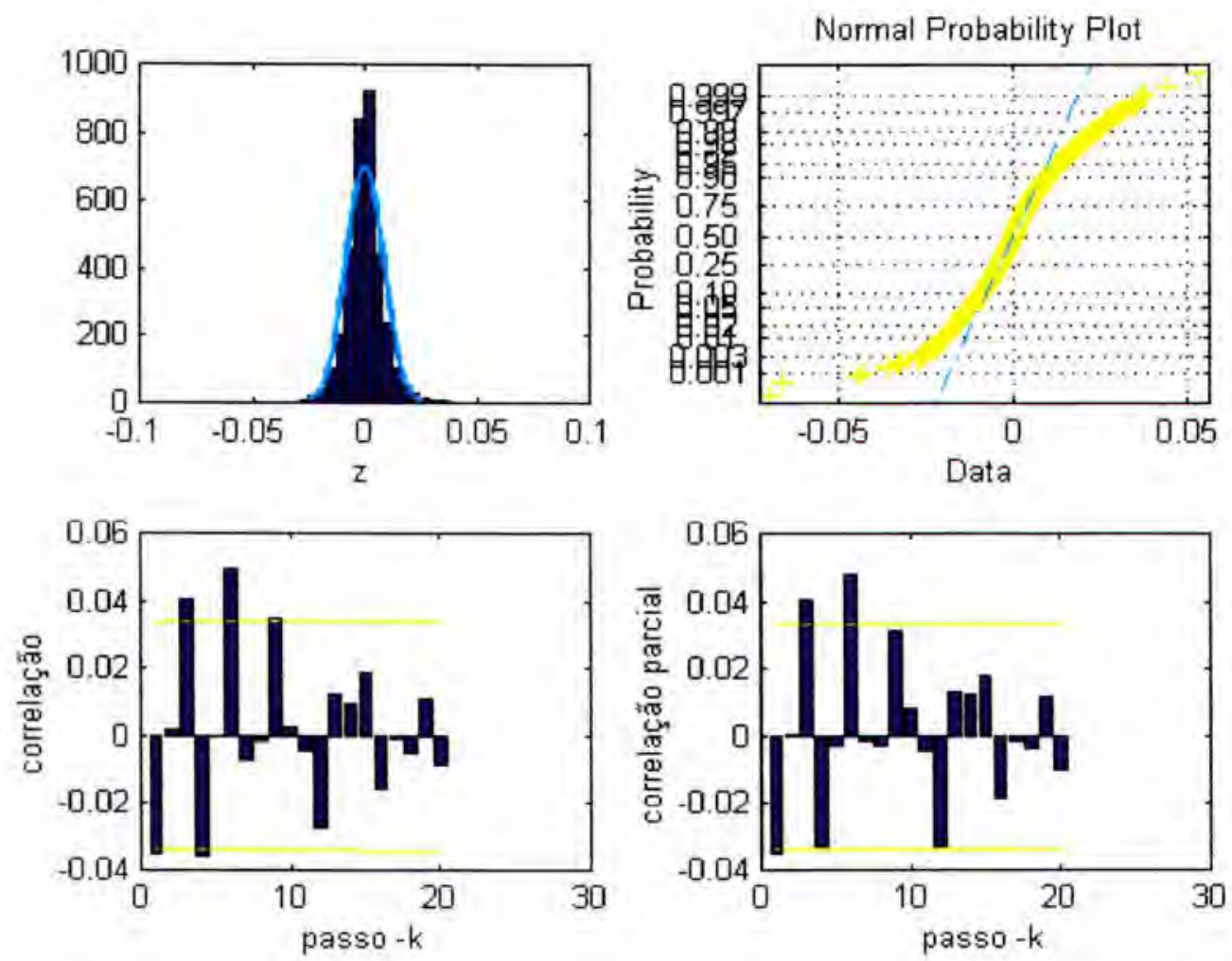

Figura 23: Histograma, gráficos normal probabilístico, auto-correlação e correlação parcial da série $z_{t}$-série moeda

Através do gráfico normal probabilístico podemos observar que a série da moeda Iene Japonês não apresenta distribuição normal. Podemos observar também uma pequena correlação entre os valores da série $z_{t}$.

Tabela 7: Critérios de seleção de modelos

\begin{tabular}{lccc}
\hline \multicolumn{4}{c}{ ARCH } \\
\hline Ordem do Modelo & $\mathbf{6}$ & $\mathbf{7}$ & $\mathbf{8}$ \\
Log Verossimilhança & $1.2115 \mathrm{e}+004$ & $1.2125 \mathrm{e}+004$ & $1.2133 \mathrm{e}+004$ \\
AIC & -6.95 & -6.9641 & -6.9681 \\
BIC & -6.94 & -6.9500 & -6.9522 \\
CPO & $2.5591 \mathrm{e}+047$ & $2.3866 \mathrm{e}+047$ & $2.8471 \mathrm{e}+047$ \\
\hline
\end{tabular}

Pelos critérios AIC/BIC e CPO o modelo escolhido foi o ARCH de ordem 8. 
Tabela 8: Estimativas dos parâmetros do modelo $\mathrm{ARCH}(8)$

\begin{tabular}{lllllll}
\hline & \multicolumn{3}{c}{ M.V } & \multicolumn{4}{c}{ Bayesiano } \\
\cline { 2 - 7 } & $\hat{\boldsymbol{\alpha}}$ & $\begin{array}{c}\text { Intervalo de } \\
\text { Confiança }\end{array}$ & Média & Moda & $\begin{array}{c}\text { Desvio } \\
\text { Padrão }\end{array}$ & $\begin{array}{c}\text { Intervalo de } \\
\text { Credibilidade }\end{array}$ \\
\hline$\alpha_{0}$ & 0.00001 & $(0.000010 ; 0.000015)$ & 0.000012 & 0.000012 & 0.0000013 & $(0.000010 ; 0.000015)$ \\
$\alpha_{1}$ & 0.2004 & $(0.15062 ; 0.25031)$ & 0.1987 & 0.1999 & 0.0274 & $(0.1491 ; 0.2563)$ \\
$\alpha_{2}$ & 0.1536 & $(0.10791 ; 0.19946)$ & 0.1532 & 0.1439 & 0.0247 & $(0.1082 ; 0.2048)$ \\
$\alpha_{3}$ & 0.1499 & $(0.10407 ; 0.19586)$ & 0.1515 & 0.1628 & 0.0256 & $(0.1031 ; 0.2013)$ \\
$\alpha_{4}$ & 0.0819 & $(0.04341 ; 0.12043)$ & 0.0872 & 0.0808 & 0.0181 & $(0.0573 ; 0.1255)$ \\
$\alpha_{5}$ & 0.1053 & $(0.06425 ; 0.14643)$ & 0.1111 & 0.0891 & 0.0228 & $(0.0677 ; 0.1600)$ \\
$\alpha_{6}$ & 0.0858 & $(0.04724 ; 0.12444)$ & 0.0931 & 0.0752 & 0.0231 & $(0.0576 ; 0.1445)$ \\
$\alpha_{7}$ & 0.0701 & $(0.03384 ; 0.10641)$ & 0.0807 & 0.0945 & 0.0184 & $(0.0455 ; 0.1104)$ \\
$\alpha_{8}$ & 0.0800 & $(0.04333 ; 0.11686)$ & 0.0854 & 0.0818 & 0.0193 & $(0.0500 ; 0.1287)$ \\
\hline & & & & & &
\end{tabular}

Pela tabela 8 podemos observar que as estimativas via máxima verossimilhança e bayesianas e os intervalos de confiança e credibilidade apresentaram-se bem próximos.

$\mathrm{Na}$ implementação do algoritmo Metrópolis-Hastings utilizamos o mesmo procedimento da série Telebrás. O critério de convergência de Gelman e Rubin foi verificado para todos os parâmetros.

A figura 24 apresenta os histogramas construídos com as amostras selecionadas dos parâmetros do modelo.
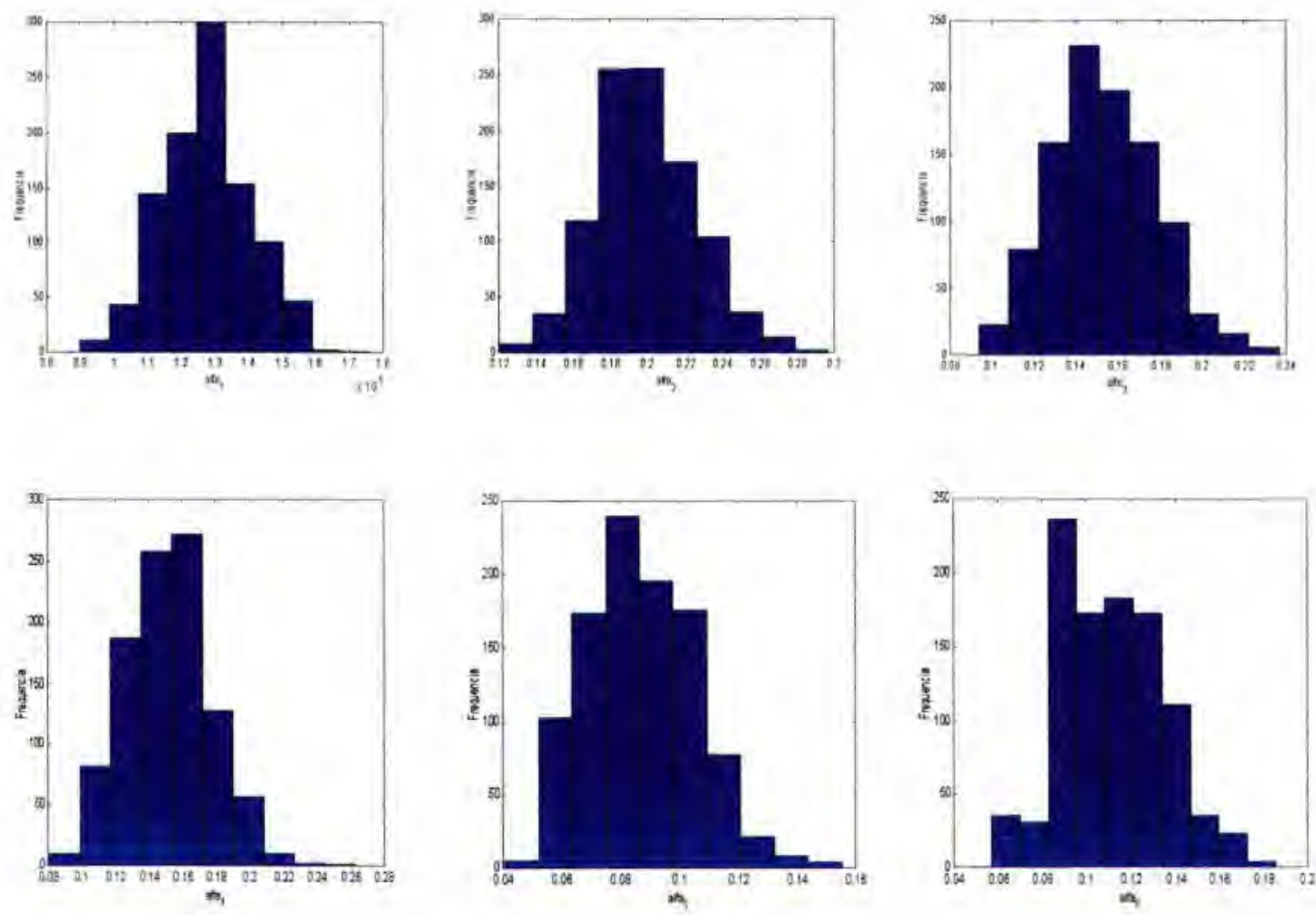

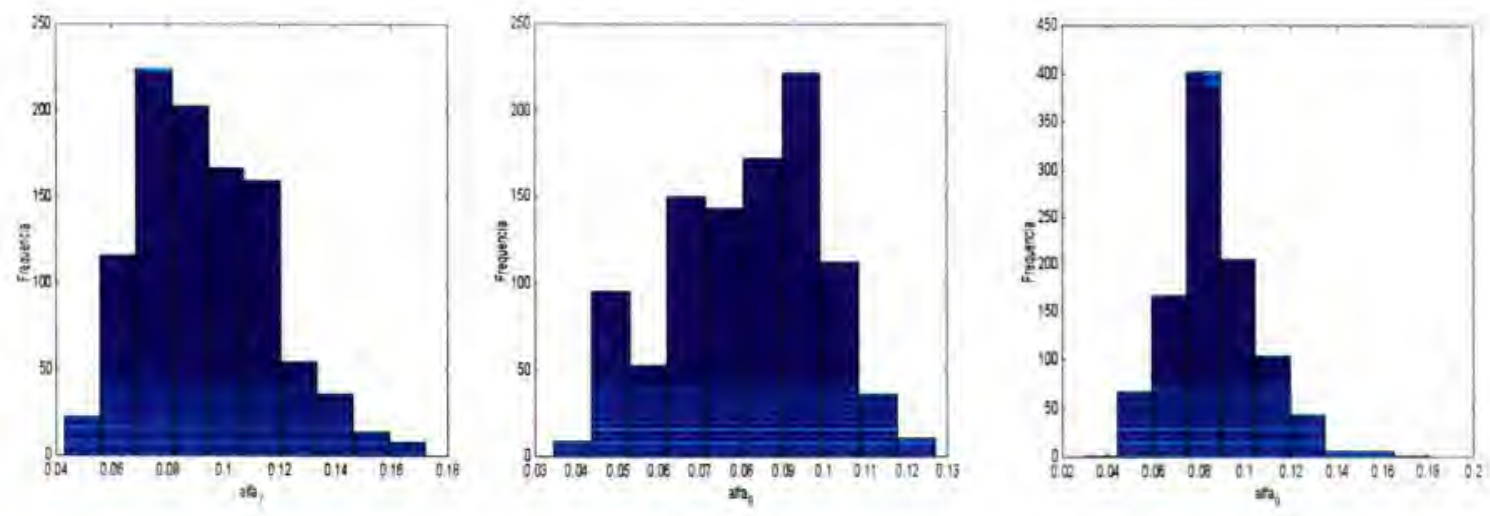

Figura 24: Distribuição a Posteriori Marginal dos parâmetros do modelo - série moeda
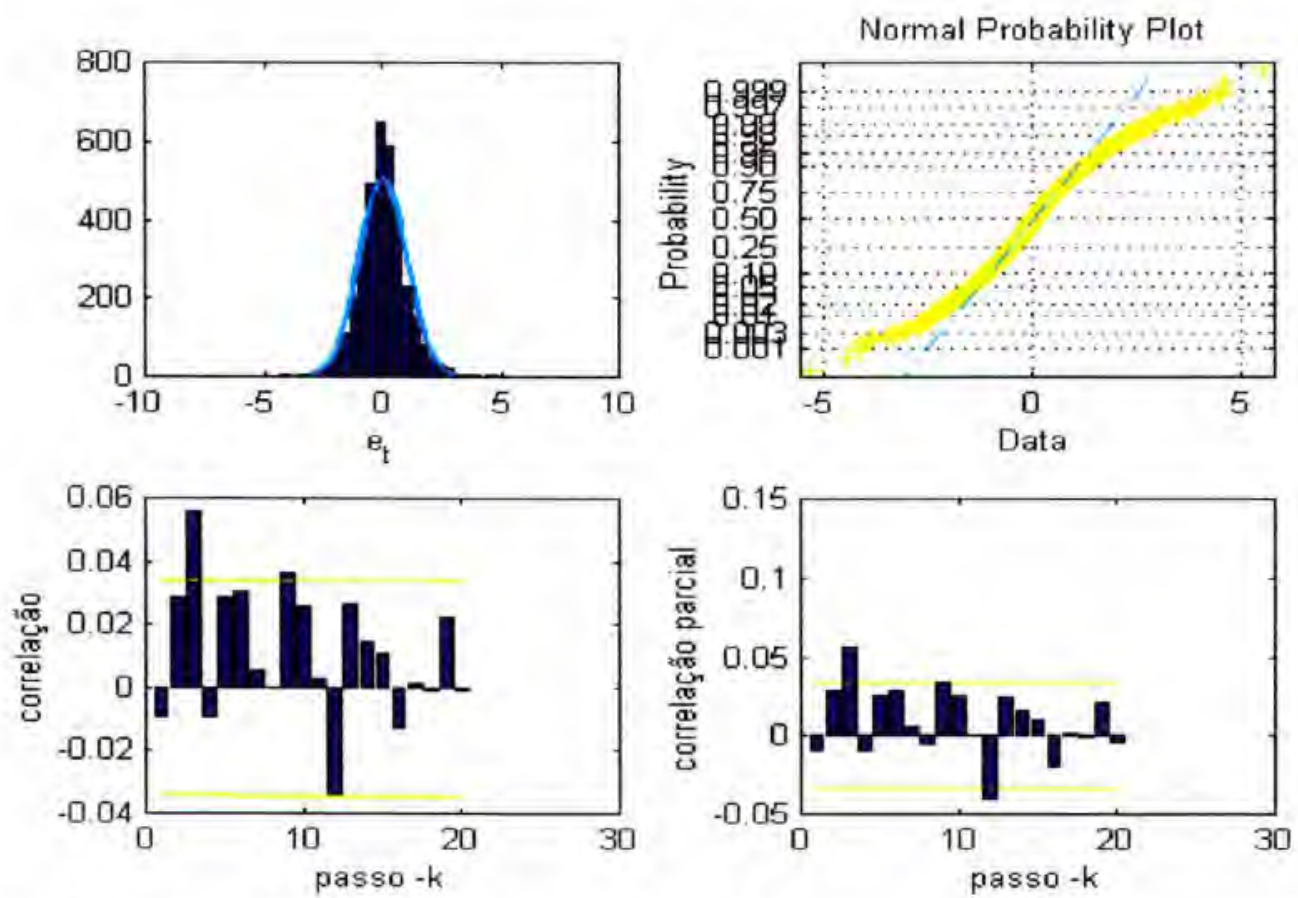

Figura 25: Histograma, gráficos normal probabilístico, auto-correlação e correlação parcial dos resíduos - série moeda

A análise de resíduos mostrada na figura 25 indica que a distribuição dos resíduos não é normal e que os mesmos apresentam uma pequena correlação. 


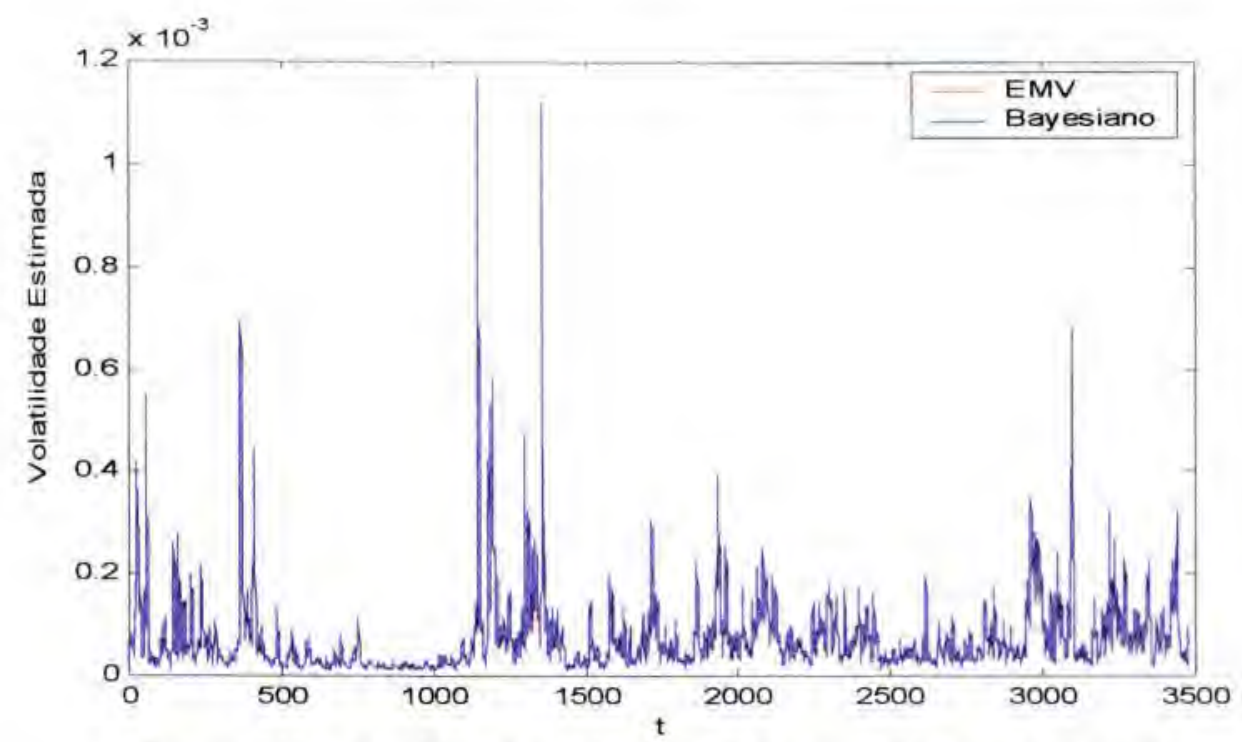

Figura 26: Gráfico da volatilidade estimada - série moeda

O gráfico da figura 26 foi feito utilizando o mesmo procedimento das séries anteriores.

A estimativa da volatilidade corresponde a um período de 3480 dias.

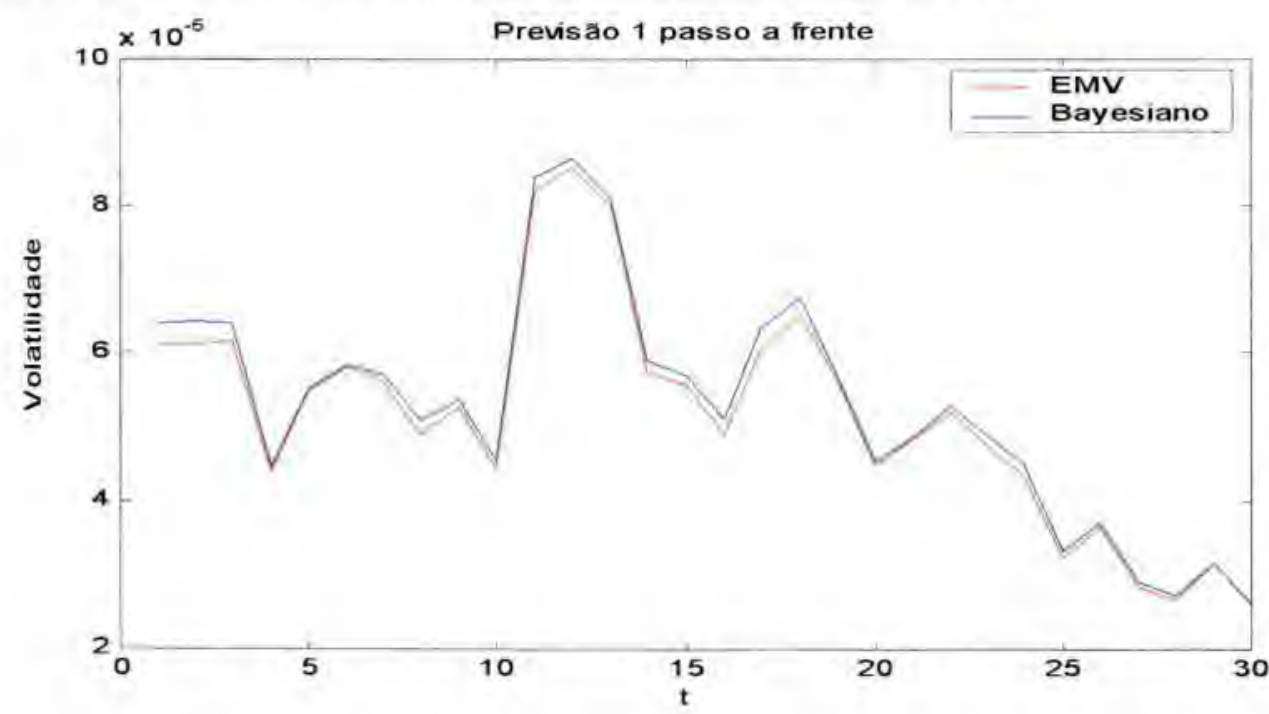

Figura 27: Previsão da volatilidade 1 passo a frente - série moeda

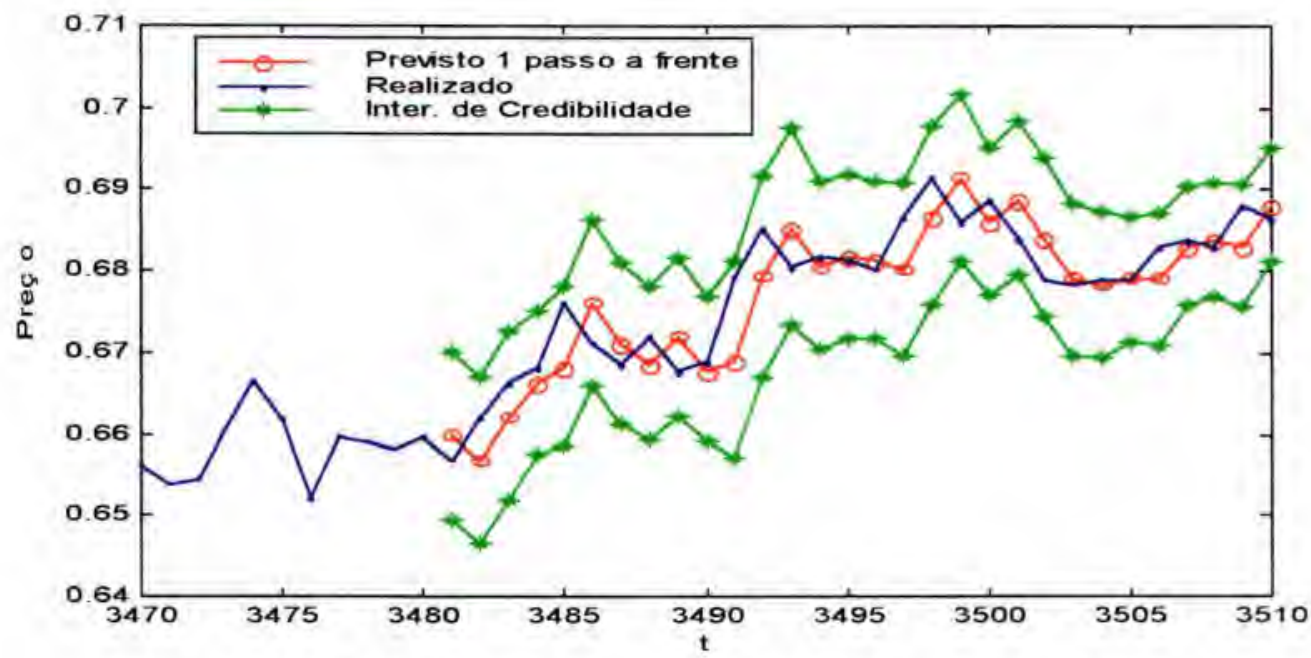

Figura 28: Previsão do preço 1 passo a frente - série moeda 
As figuras 27 e 28 apresentam as previsões, um passo a frente, da volatilidade e do preço durante um período de 30 dias.

A previsão da volatilidade e do preço, para todas as séries estudadas, foram avaliadas através da função perda Erro Médio Quadrático (EMQ). Calculamos também o Erro Médio Percentual (EMP) para a previsão do preço. Os valores obtidos estão na tabela 9 .

Tabela 9: Avaliação da previsão da volatilidade e do preço-1 passo a frente

\begin{tabular}{lccc}
\hline & Volatilidade & \multicolumn{2}{c}{ Preço } \\
\cline { 2 - 4 } & EMQ & EMQ & EMP(\%) \\
\hline Série Gerada & $2.9091 \mathrm{e}-005$ & 0.0055 & 5.2115 \\
Série lbovespa & $7.8280 \mathrm{e}-006$ & 0.2588 & 3.6415 \\
Série Telebrás & $3.1763 \mathrm{e}-005$ & $1.8237 \mathrm{e}-006$ & 2.1833 \\
Série Moeda & $3.5004 \mathrm{e}-008$ & $1.7973 \mathrm{e}-005$ & 0.5133 \\
\hline
\end{tabular}

\subsection{Resultados obtidos com o ajuste do modelo $\operatorname{GARCH}(p, q)$}

$\mathrm{Na}$ análise clássica e bayesiana dos modelos $\operatorname{GARCH}(\mathrm{p}, \mathrm{q})$ ajustamos, tanto para a série gerada quanto para as séries reais, o modelo $\operatorname{GARCH}(1,1)$. Todas as análises foram feitas sob a suposição de normalidade.

\subsubsection{Série Gerada}

Esta série consiste de 500 observações geradas por um $\operatorname{GARCH}(1,1)$. A análise com esta série foi feita com a finalidade de validar os algoritmos desenvolvidos. A figura 29 apresenta os retornos do logaritmo da série.

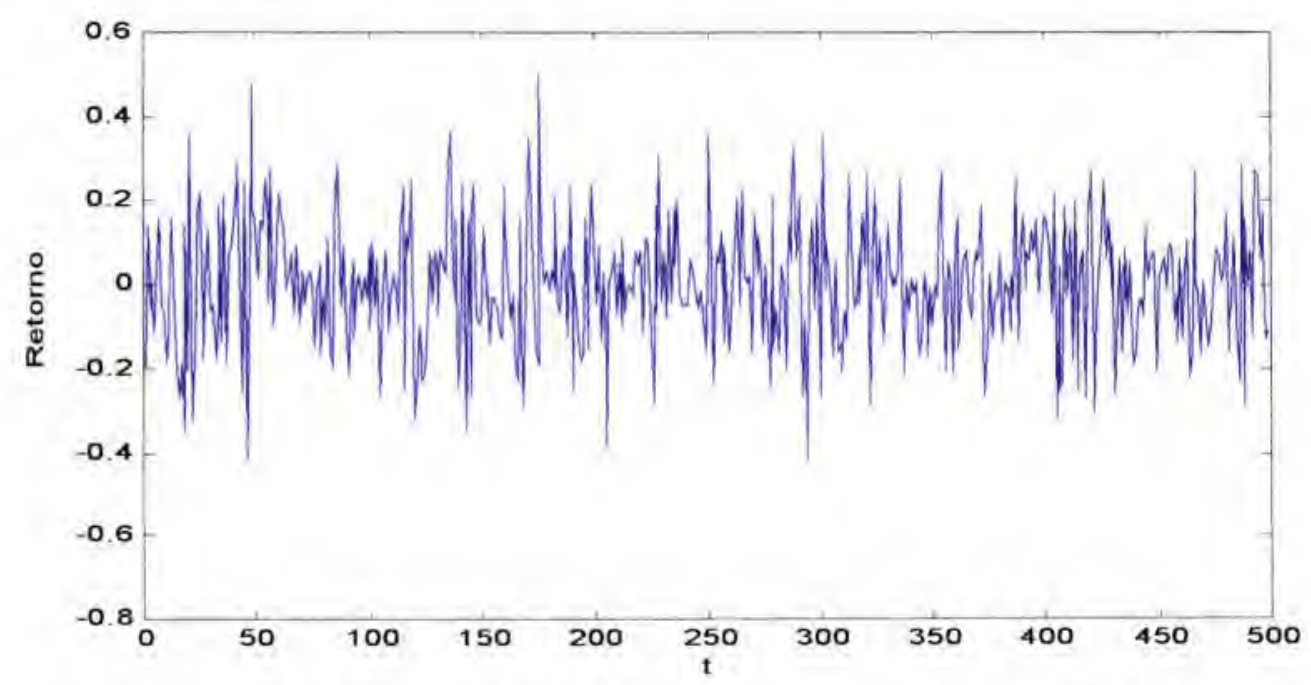

Figura 29: GARCH(1,1) com $\alpha_{0}=0.05, \alpha_{1}=0.3$ e $\beta_{1}=0.5$ 

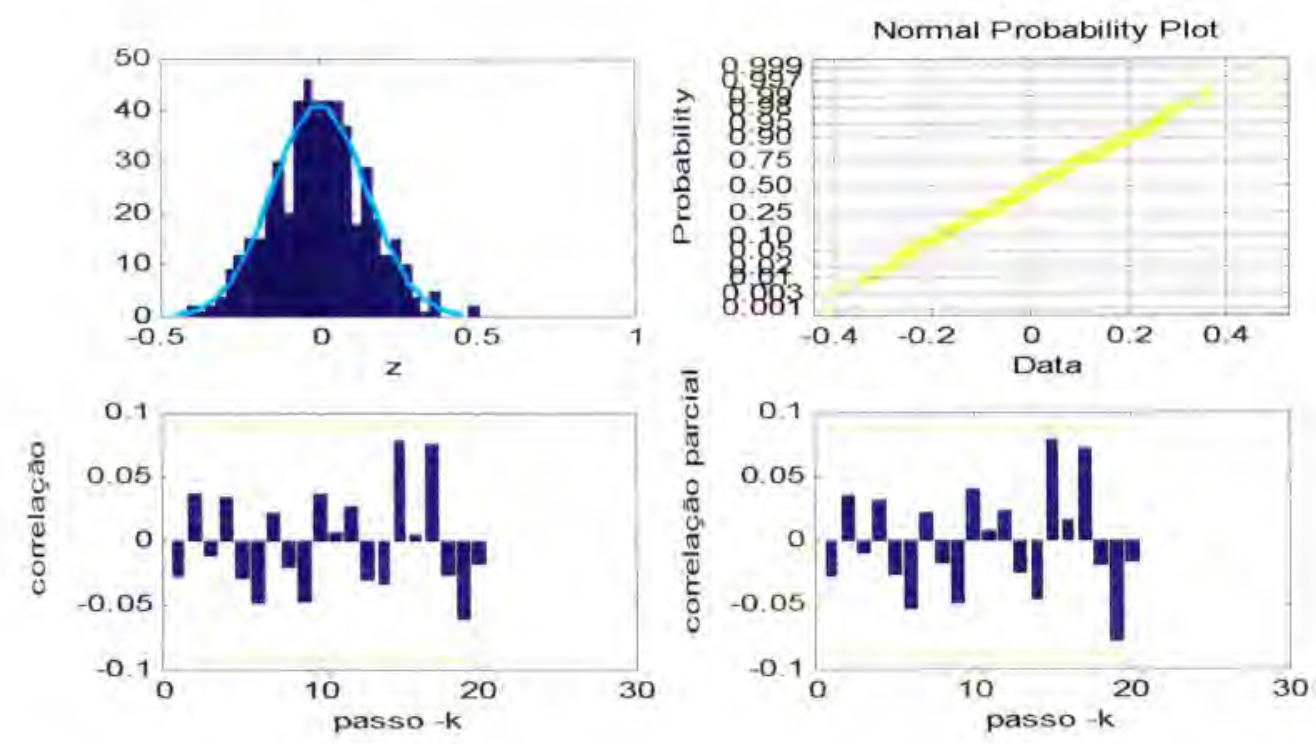

Figura 30: Histograma, gráficos normal probabilístico, auto-correlação e correlação parcial da série $z_{t}$ - série gerada

Pelos gráficos acima observamos que a série apresenta distribuição normal e os gráficos da correlação e da correlação parcial indicam que a série $z_{t}$ não apresenta correlação.

As estimativas dos parâmetros obtidas via máxima verossimilhança e MCMC utilizando o algoritmo Metropolis-Hastings estão apresentadas na tabela 10.

Tabela 10: Estimativas dos parâmetros do modelo GARCH(1,1)

\begin{tabular}{lccccccc}
\hline & \multicolumn{3}{c}{ M.V } & \multicolumn{4}{c}{ Bayesiano } \\
\cline { 2 - 8 } & $\hat{\boldsymbol{\theta}}$ & $\begin{array}{c}\text { Intervalo de } \\
\text { Confiança }\end{array}$ & Média & Moda & Mediana & $\begin{array}{c}\text { Desvio } \\
\text { Padrão }\end{array}$ & $\begin{array}{c}\text { Intervalo de } \\
\text { Credibilidade }\end{array}$ \\
\hline$\alpha_{0}$ & 0.0050 & $(0.0011 ; 0.0088)$ & 0.0068 & 0.0068 & 0.0065 & 0.0018 & $(0.0039 ; 0.0110)$ \\
$\alpha_{1}$ & 0.2425 & $(0.1110 ; 0.3739)$ & 0.2869 & 0.2903 & 0.2846 & 0.0751 & $(0.1486 ; 0.4519)$ \\
$\beta_{1}$ & 0.5425 & $(0.3045 ; 0.7805)$ & 0.4398 & 0.4738 & 0.4446 & 0.0984 & $(0.2453 ; 0.6366)$ \\
\hline
\end{tabular}

As estimativas de máxima verossimilhança e bayesianas se aproximaram dos verdadeiros valores dos parâmetros e o intervalo de credibilidade mostrou-se mais preciso, exceto para o parâmetro $\alpha_{1}$.

$\mathrm{Na}$ implementação do algoritmo Metrópolis-Hastings, para cada parâmetro, foram simuladas cinco cadeias com 3.000 iterações cada, descartadas $50 \%$ de cada uma e tomados valores espaçados de 15 em 15 totalizando uma amostra de tamanho 500 .

O critério de Gelman e Rubin foi verificado, obtendo-se $\sqrt{\hat{R}}<1.2$ para todos os parâmetros. 
Nos gráficos da figura 31 apresentamos os histogramas construídos com as amostras selecionadas para os parâmetros $\alpha_{0}, \alpha_{1}$ e $\beta_{1}$, respectivamente.
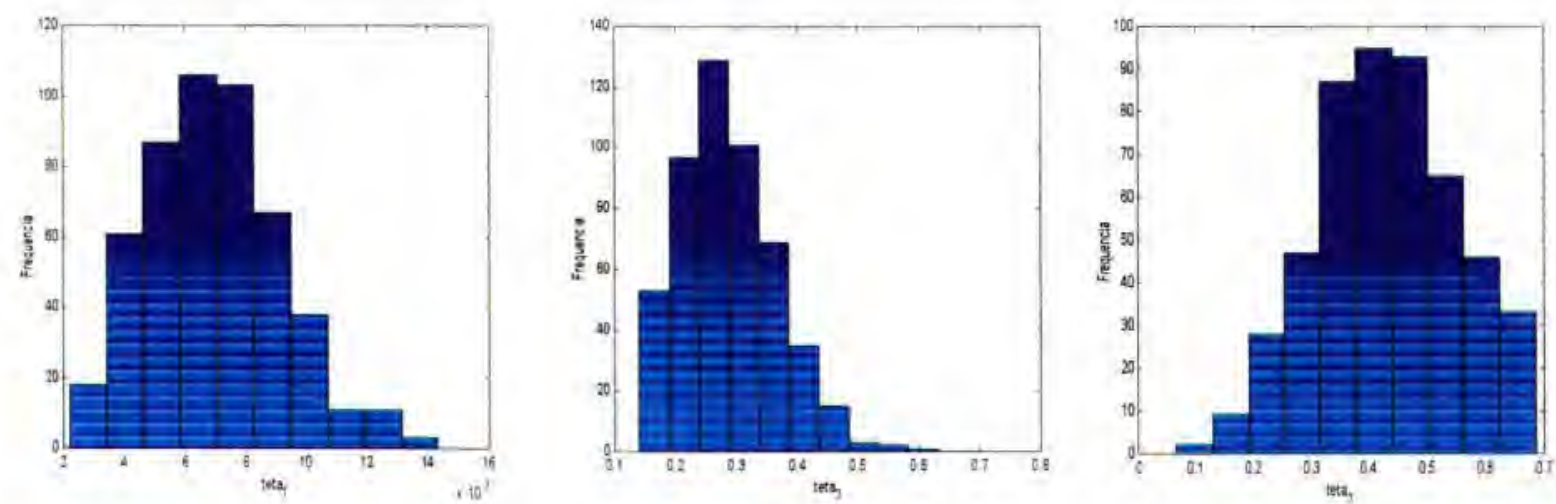

Figura 31: Distribuição a Posteriori Marginal: $\alpha_{0}, \alpha_{1}$ e $\beta_{1}$
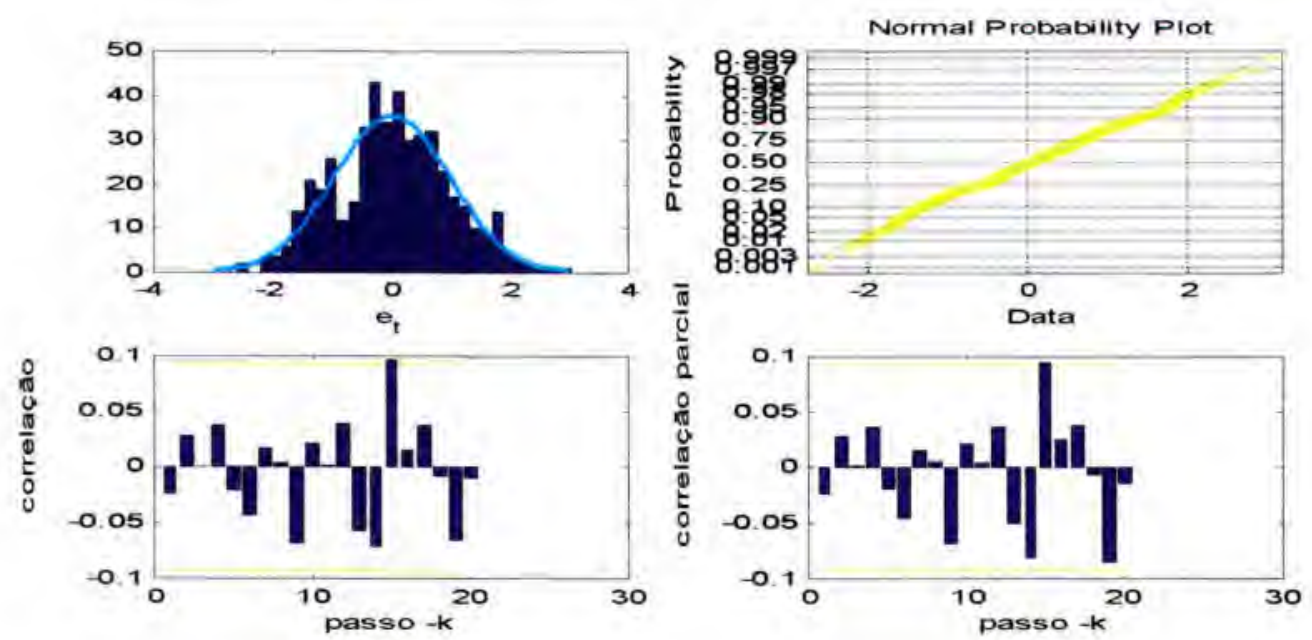

Figura 32: Histograma, gráficos normal probabilístico, auto-correlação e correlação parcial dos resíduos- série gerada

Os gráficos da figura 32 resumem a análise dos resíduos para o modelo ajustado na abordagem bayesiana. Observamos que os residuos estão normalmente distribuídos e não apresentam correlação significativa.

A figura 33 apresenta o gráfico da volatilidade estimada para um período de 470 dias. Os últimos 30 dias foram deixados fora do ajuste para serem utilizados no cálculo da previsão da volatilidade e do preço. 


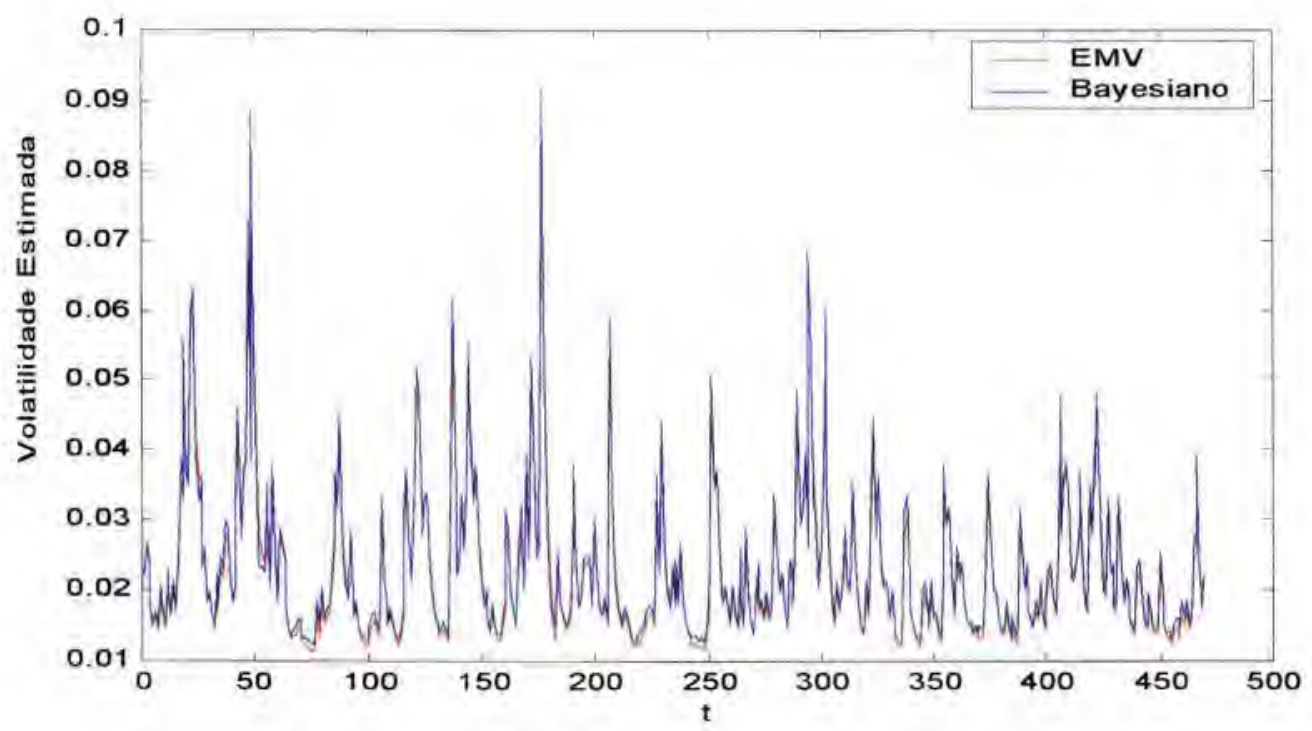

Figura 33: Gráfico da volatilidade estimada - série gerada

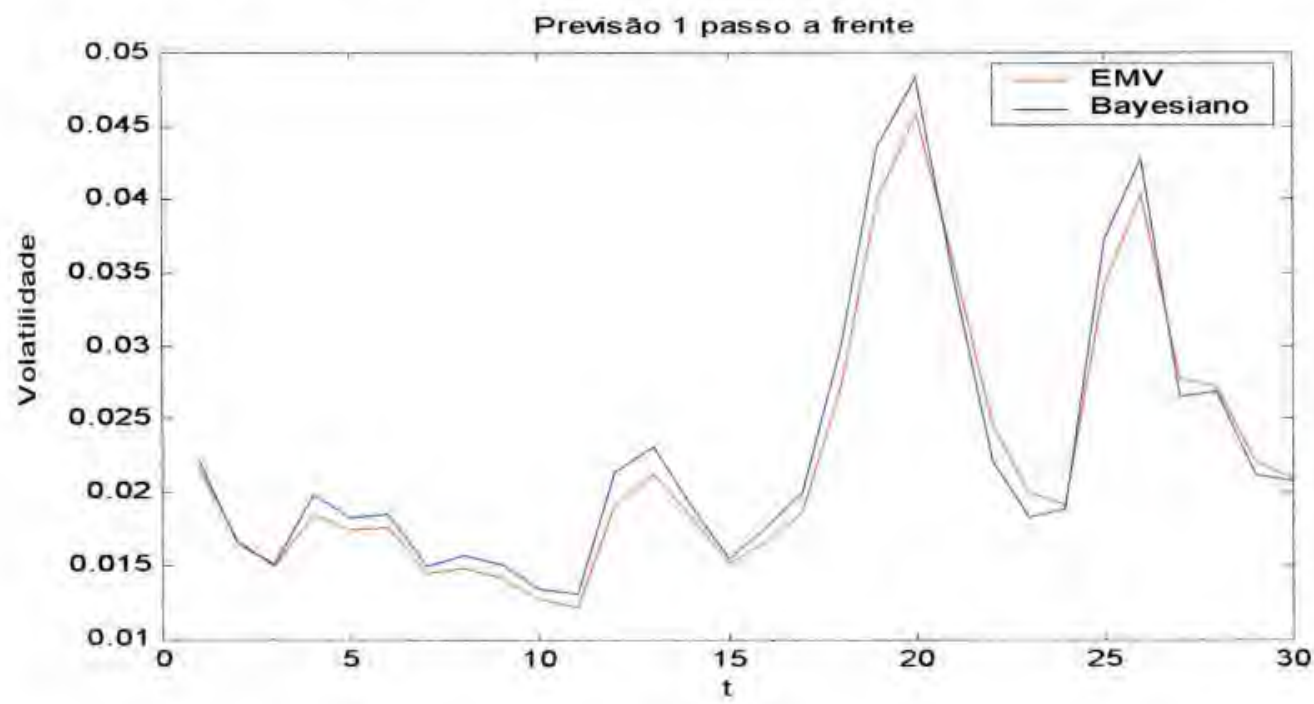

Figura 34: Previsão da volatilidade 1 passo a frente - série gerada

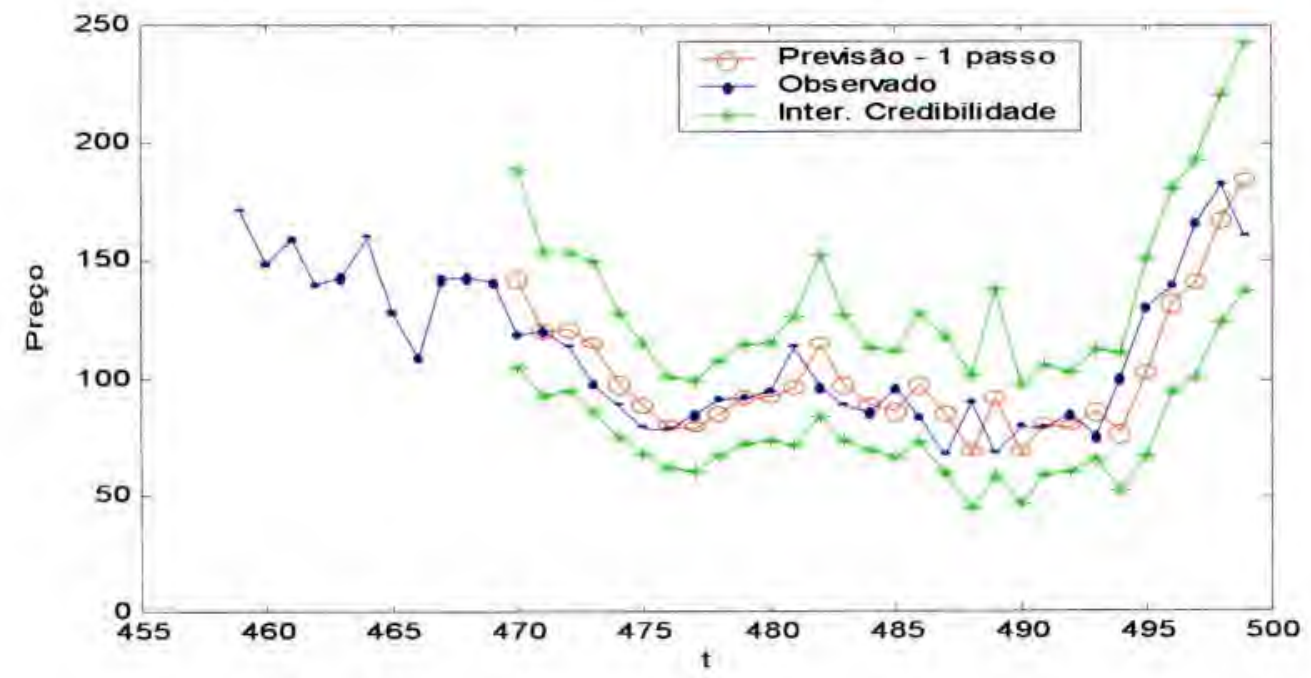

Figura 35: Previsão do preço 1 passo a frente - série gerada 
As figuras 34 e 35 apresentam as previsões, um passo à frente, da volatilidade e do preço durante um período de 30 dias.

\subsubsection{Série Índice Bovespa}

Esta série é a mesma que utilizamos no ajuste do modelo $\mathrm{ARCH}(\mathrm{p})$. Portanto, o gráfico dos retornos e o gráfico normal probabilístico são os mesmos apresentados na seção 5.1.1. A tabela 11 fornece os valores da função de verossimilhança e os valores dos critérios de seleção de modelos no ajuste do $\operatorname{GARCH}(1,1)$ e do modelo $\operatorname{ARCH}(3)$ ajustado na seção 5.1.1. Podemos observar que pelo critério AIC/BIC o modelo GARCH(1,1) é mais adequado e pelo critério da densidade preditiva ordenada o modelo escolhido é o $\mathrm{ARCH}(3)$.

Tabela 11: Critérios de seleção de modelos

\begin{tabular}{lcc}
\hline & GARCH & ARCH \\
\hline Ordem do Modelo & $(1,1)$ & 3 \\
Log Verossmilhança & $1.28 e+003$ & $1.27 e+003$ \\
AIC & -4.1343 & -4.0990 \\
BIC & -4.1129 & -4.0705 \\
CPO & $5.59 e+013$ & $2.74 \mathrm{e}+017$ \\
\hline
\end{tabular}

As estimativas dos parâmetros obtidas via máxima verossimilhança e MCMC utilizando o algoritmo Metropolis-Hastings estão apresentadas na tabela 12.

Tabela 12: Estimativas dos parâmetros do modelo GARCH(1,1)

\begin{tabular}{cccccccc}
\hline & \multicolumn{3}{c}{ M.V } & \multicolumn{5}{c}{ Bayesiano } \\
\cline { 2 - 8 } & $\hat{\boldsymbol{\theta}}$ & $\begin{array}{c}\text { Intervalo de } \\
\text { Confiança }\end{array}$ & Média & Moda & Mediana & $\begin{array}{c}\text { Desvio } \\
\text { Padräo }\end{array}$ & $\begin{array}{c}\text { Intervalo de } \\
\text { Credibilidade }\end{array}$ \\
\hline$\alpha_{0}$ & 0.00005 & $\begin{array}{c}(0.00002368 ; 0.0000 \\
9204)\end{array}$ & 0.00007 & 0.00007 & 0.00007 & $1.503 \mathrm{e}-009$ & $(0.00006999 ; 0.000$ \\
$\alpha_{1}$ & 0.1610 & $(0.0976 ; 0.2243)$ & 0.1501 & 0.1500 & 0.1501 & $6.686 \mathrm{e}-004$ & $(0.1487 ; 0.1514)$ \\
$\beta_{1}$ & 0.8025 & $(0.7351 ; 0.8699)$ & 0.7505 & 0.7508 & 0.7505 & $1.263 \mathrm{e}-003$ & $(0.7479 ; 0.7529)$ \\
\hline
\end{tabular}

As estimativas de máxima verossimilhança e bayesianas apresentaram-se próximas e o intervalo de credibilidade mostrou-se mais preciso.

$\mathrm{Na}$ implementação do algoritmo Metrópolis-Hastings utilizamos o mesmo procedimento aplicado na série gerada. 
O critério de Gelman e Rubin foi verificado, obtendo-se $\sqrt{\hat{R}}<1.2$ para todos os parâmetros.

Nos gráficos da figura 36 apresentamos os histogramas construidos com as amostras selecionadas para os parâmetros $\alpha_{0}, \alpha_{1}$ e $\beta_{1}$, respectivamente.
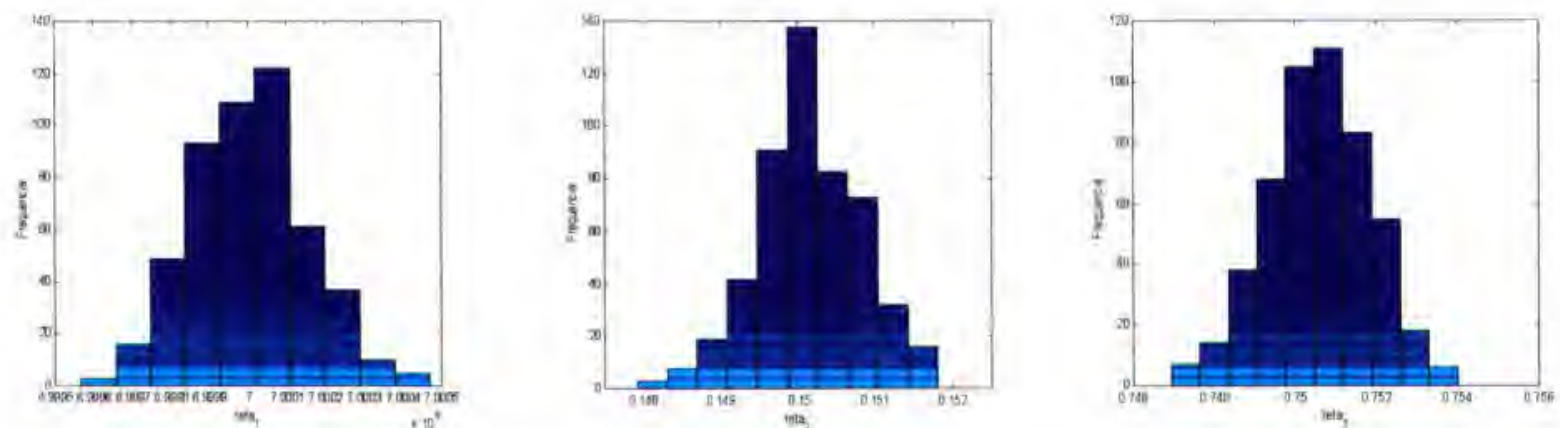

Figura 36: Distribuição a Posteriori Marginal: $\alpha_{0}, \alpha_{1}$ e $\beta_{1}$
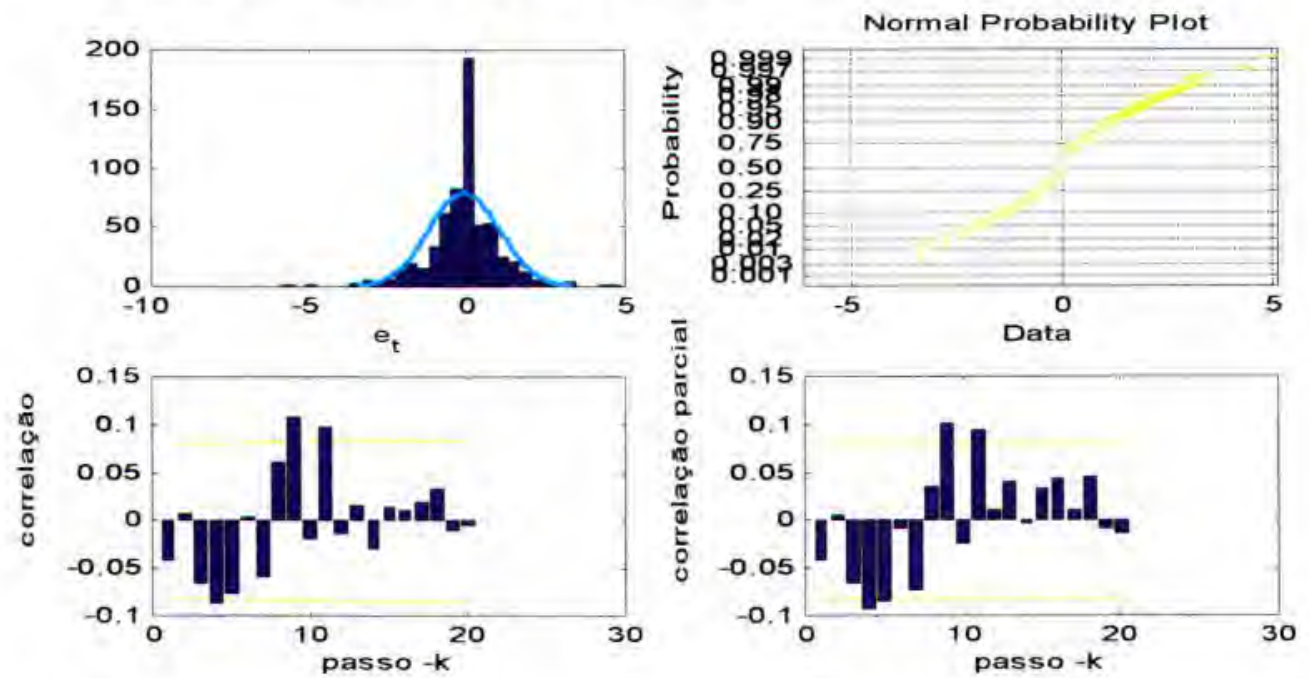

Figura 37: Histograma, gráficos normal probabilístico, auto-correlação e correlação parcial dos resíduos- série ibovespa

Através dos gráficos da figura 37 podemos verificar que a distribuição dos resíduos não é normal e apresenta uma pequena correlação.

A figura 38 apresenta o gráfico da volatilidade estimada para um periodo de 621 dias. Os últimos 30 dias foram deixados fora do ajuste para serem utilizados no cálculo da previsão da volatilidade e do preço. 


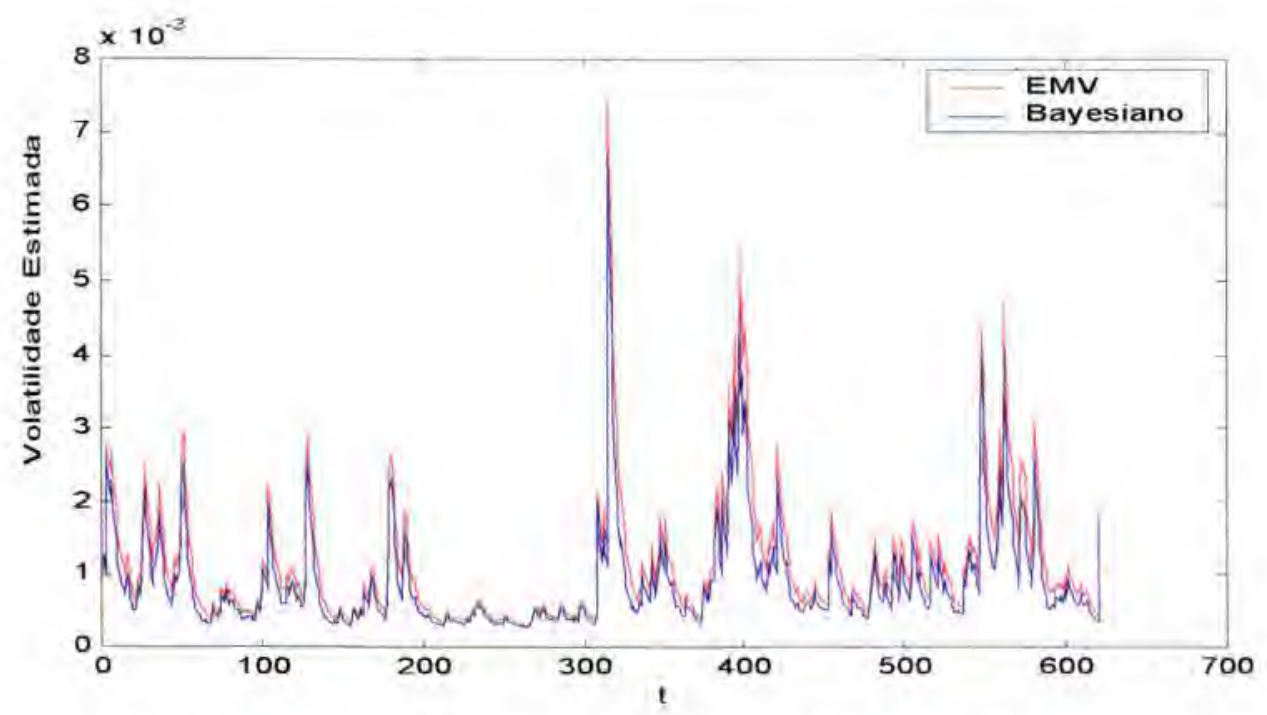

Figura 38: Gráfico da volatilidade estimada - série ibovespa

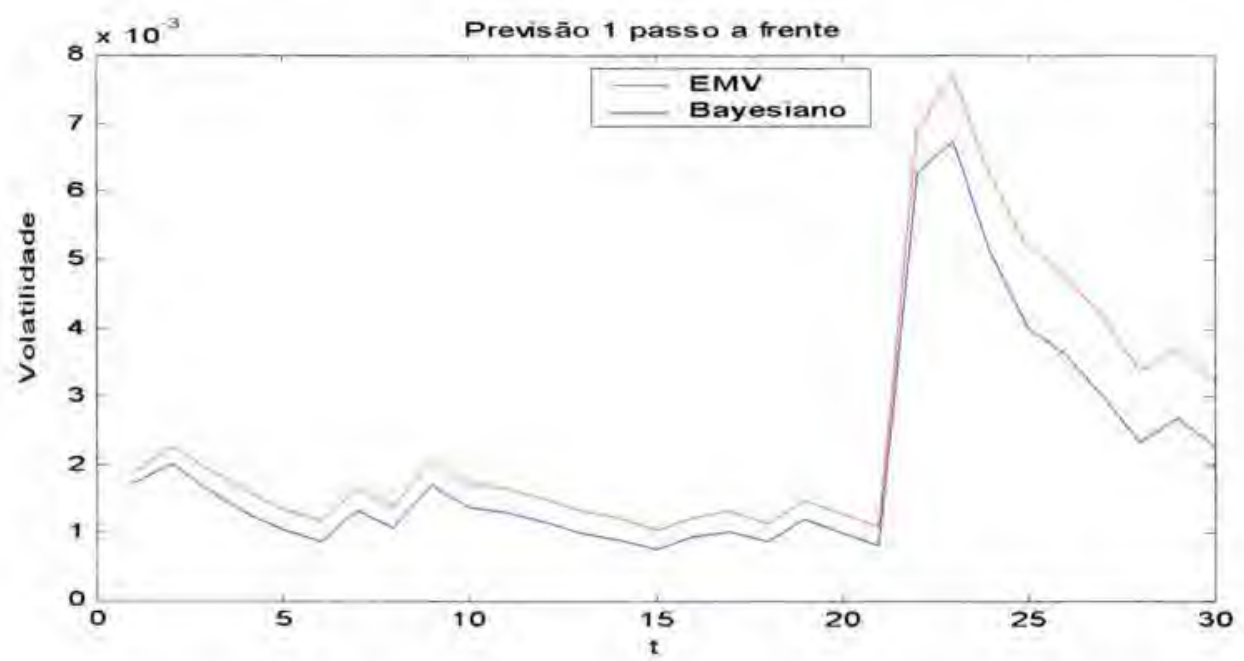

Figura 39: Previsão da volatilidade 1 passo a frente - série ibovespa

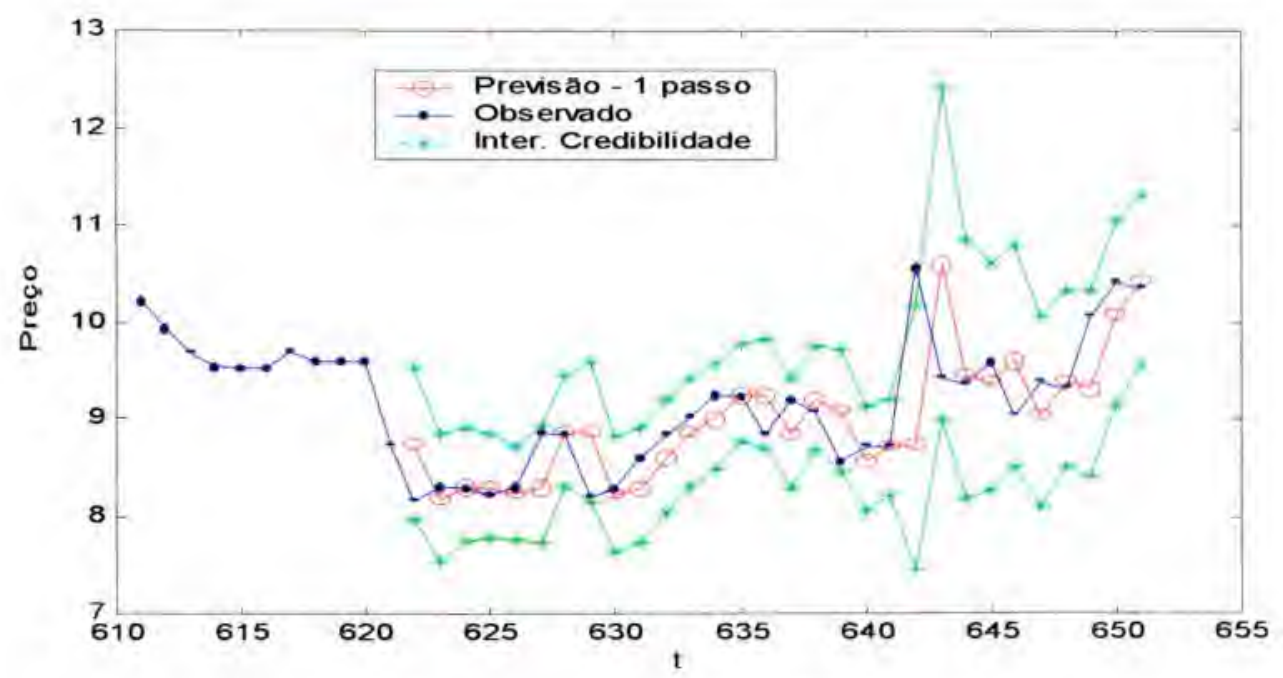

Figura 40: Previsão do preço 1 passo a frente - série ibovespa 
As figuras 39 e 40 apresentam a previsão um passo à frente da volatilidade e do preço, respectivamente, durante um período de 30 dias. A previsão do preço apresentada foi feita com os resultados obtidos na abordagem bayesiana.

\subsubsection{Série Telebrás}

Os gráficos do retorno e normal probabilístico para a série Telebrás estão apresentados na seção 5.1.3. A tabela 13 fornece os valores da função de verossimilhança e os valores dos critérios de seleção de modelos no ajuste do $\operatorname{GARCH}(1,1)$ e do modelo $\operatorname{ARCH}(7)$ ajustado na seção 5,1.3. Podemos observar que pelo critério $\operatorname{AIC/BIC~o~modelo~} \operatorname{GARCH}(1,1)$ é mais adequado e pelo critério da densidade preditiva ordenada o modelo escolhido é o ARCH(7).

Tabela 13: Critérios de seleção de modelos

\begin{tabular}{lcc}
\hline & GARCH & ARCH \\
\hline Ordem do Modelo & $\mathbf{( 1 , 1 )}$ & $\mathbf{7}$ \\
Log Verossmilhança & $1.62 \mathrm{e}+003$ & $1.62 \mathrm{e}+003$ \\
AIC & -3.3964 & -3.3742 \\
BIC & -3.3812 & -3.3335 \\
CPO & $7.114 \mathrm{e}+026$ & $9.712 \mathrm{e}+026$ \\
\hline
\end{tabular}

As estimativas dos parâmetros obtidas via máxima verossimilhança e MCMC utilizando o algoritmo Metropolis-Hastings estão apresentadas na tabela 14.

Tabela 14: Estimativas dos parâmetros do modelo GARCH(1,1)

\begin{tabular}{cccccccc}
\hline & \multicolumn{3}{c}{ M.V } & \multicolumn{5}{c}{ Bayesiano } \\
\cline { 2 - 8 } & $\hat{\boldsymbol{\theta}}$ & $\begin{array}{c}\text { Intervalo de } \\
\text { Confiança }\end{array}$ & Média & Moda & Mediana & $\begin{array}{c}\text { Desvio } \\
\text { Padräo }\end{array}$ & $\begin{array}{c}\text { Intervalo de } \\
\text { Credibilidade }\end{array}$ \\
\hline$\alpha_{0}$ & 0.00007 & $(0.00002339 ; 0.0001)$ & 0.00007 & 0.00007 & 0.00007 & $4.847 \mathrm{e}-010$ & $(0.0000699 ; 0.0000$ \\
$\alpha_{1}$ & 0.1172 & $(0.0712 ; 0.1631)$ & 0.1001 & 0.1001 & 0.1001 & $2.005 \mathrm{e}-004$ & $(0.0997 ; 0.1005)$ \\
$\beta_{1}$ & 0.8532 & $(0.8002 ; 0.9063)$ & 0.7508 & 0.7508 & 0.7508 & $6.403 \mathrm{e}-004$ & $(0.7495 ; 0.7521)$ \\
\hline
\end{tabular}

As estimativas de máxima verossimilhança e bayesianas apresentaram-se próximas e o intervalo de credibilidade mostrou-se mais preciso.

$\mathrm{Na}$ implementação do algoritmo Metrópolis-Hastings utilizamos o mesmo procedimento aplicado na série ibovespa. 
O critério de Gelman e Rubin foi verificado, obtendo-se $\sqrt{\hat{R}}<1.2$ para todos os parâmetros.

Nos gráficos da figura 41 apresentamos os histogramas construidos com as amostras selecionadas para os parâmetros $\alpha_{0}, \alpha_{1}$ e $\beta_{1}$, respectivamente.
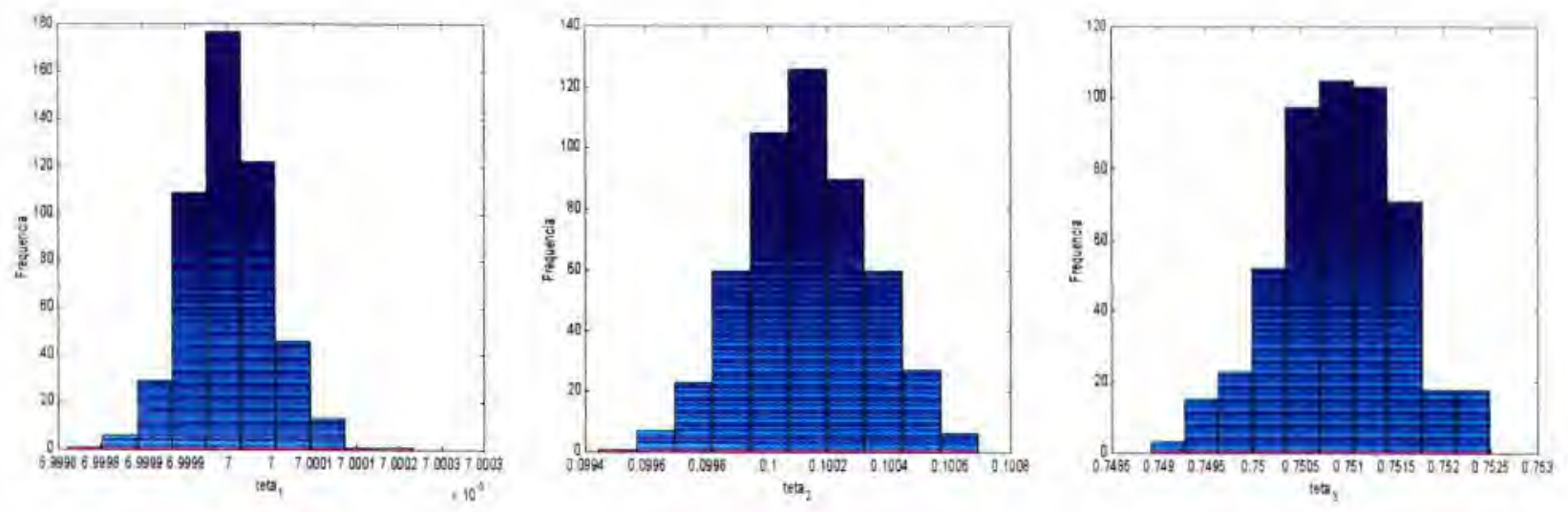

Figura 41: Distribuição a Posteriori Marginal: $\alpha_{0}, \alpha_{1}$ e $\beta_{1}$
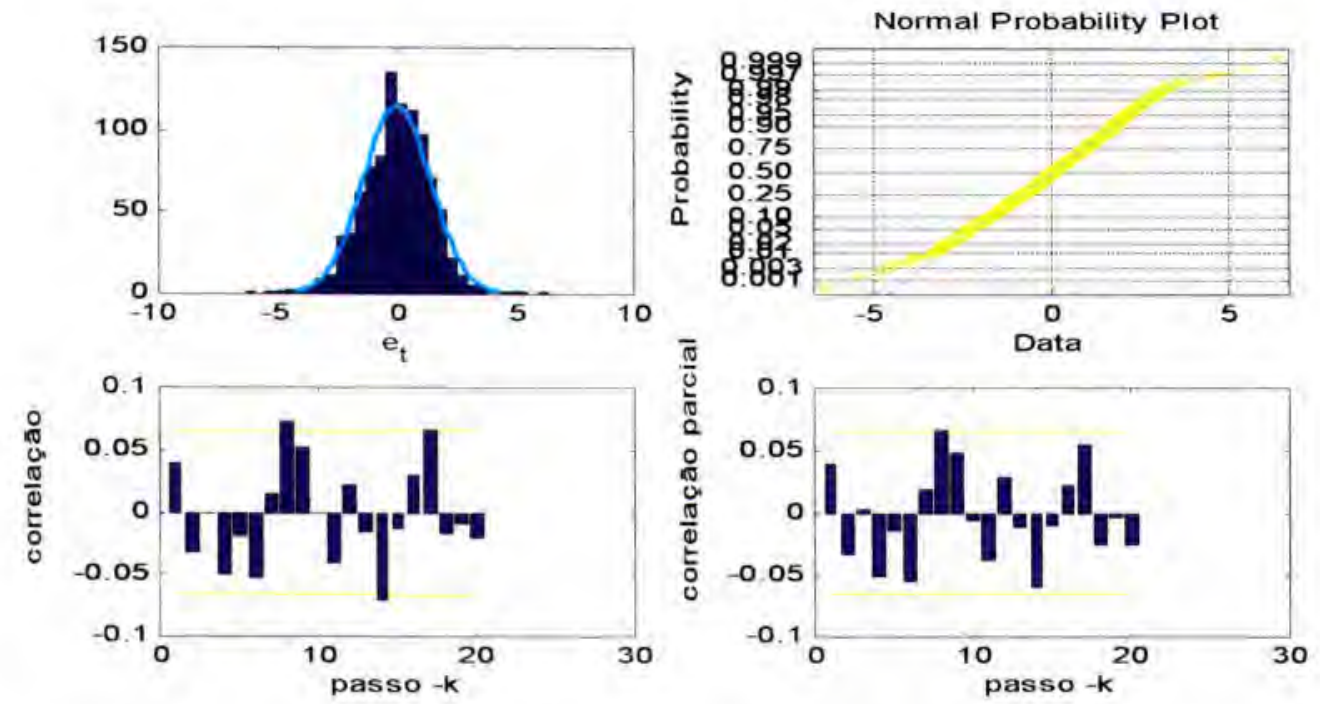

Figura 42: Histograma, gráficos normal probabilístico, auto-correlação e correlação parcial dos resíduos- série telebrás

Através da figura 42 observamos que os residuos se distanciam da normalidade nos extremos e praticamente não apresentam correlação.

A figura 43 apresenta o gráfico da volatilidade estimada para um período de 956 dias. Os últimos 30 dias foram deixados fora do ajuste para serem utilizados no cálculo da previsão da volatilidade e do preço. 


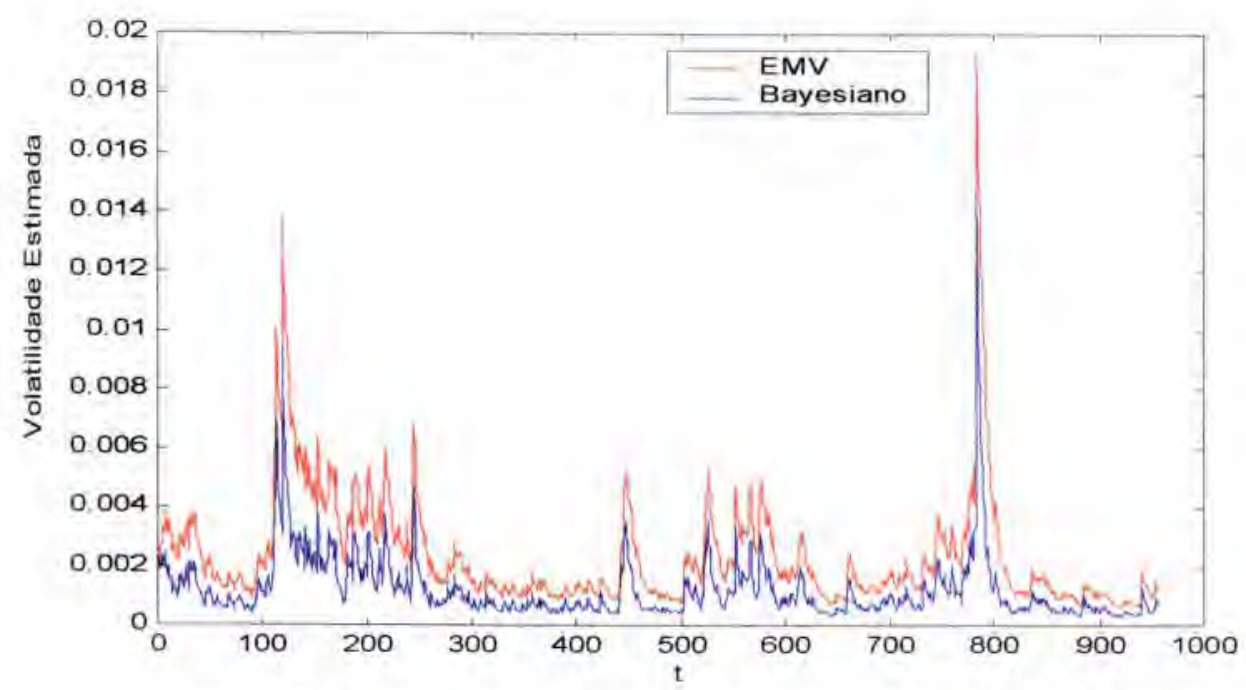

Figura 43: Gráfico da volatilidade estimada - série telebrás

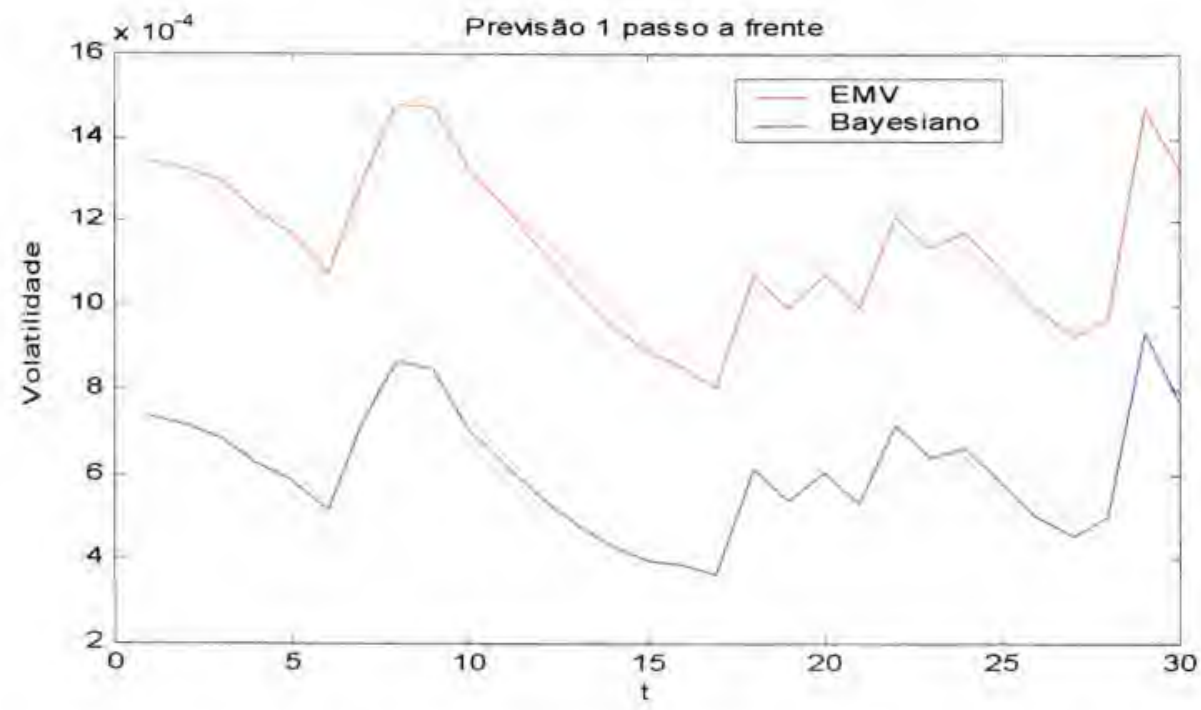

Figura 44: Previsão da volatilidade 1 passo a frente - série telebrás

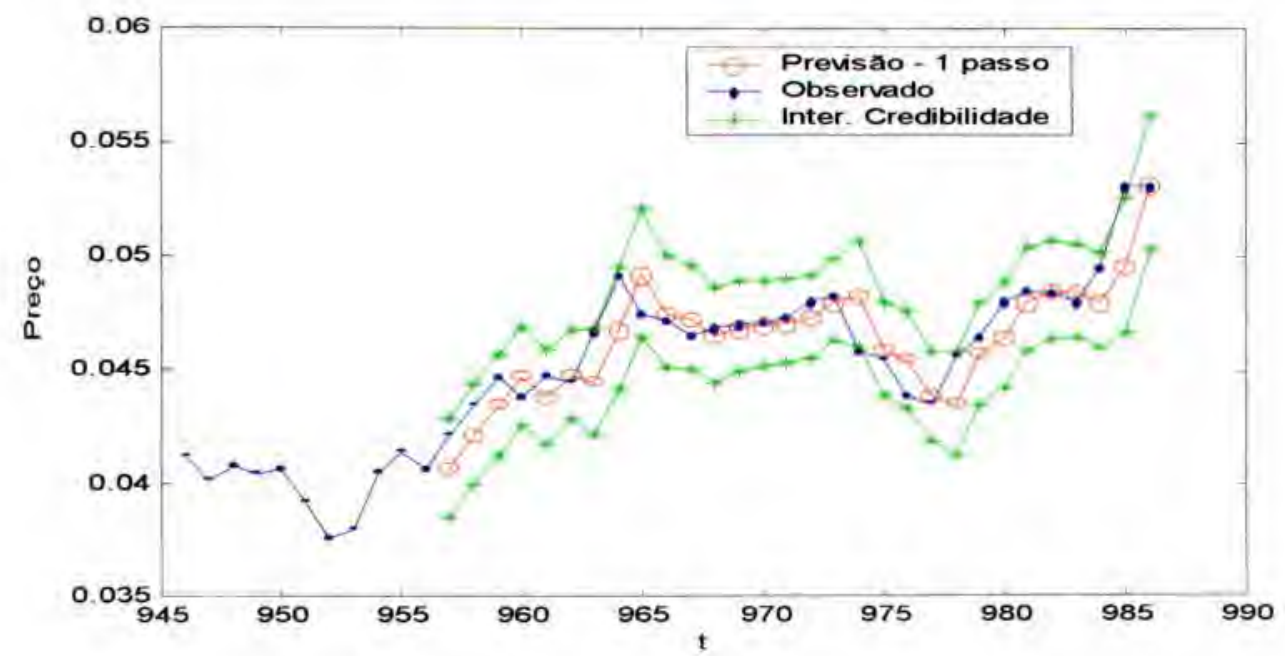

Figura 45: Previsão do preço 1 passo a frente - série telebrás 


\subsubsection{Série Cotação em Dólar Americano da moeda lene Japonês}

Os gráficos do retorno e normal probabilístico para a série moeda estão apresentados na seção 5.1.4. A tabela 14 fornece os valores dos critérios de seleção de modelos obtidos com o ajuste do GARCH(1,1) e ARCH(8). Podemos observar pelos critérios AIC/BIC e CPO que o modelo GARCH $(1,1)$ é mais adequado para o ajuste da série analisada.

Tabela 14: Critérios de seleção de modelos

\begin{tabular}{lcc}
\hline & GARCH & ARCH \\
\hline Ordem do Modelo & $(\mathbf{1 , 1 )}$ & $\mathbf{8}$ \\
Log Verossmilhança & $1.21 \mathrm{e}+004$ & $1.21 \mathrm{e}+004$ \\
AIC & -7.00 & -6.96 \\
BIC & -6.99 & -6.95 \\
CPO & $4.60 \mathrm{e}+047$ & $2.84 \mathrm{e}+047$ \\
\hline
\end{tabular}

As estimativas dos parâmetros obtidas via máxima verossimilhança e MCMC utilizando o algoritmo Metropolis-Hastings estão apresentadas na tabela 15.

Tabela 15: Estimativas dos parâmetros do modelo $\operatorname{GARCH}(1,1)$

\begin{tabular}{cccccccc}
\hline & \multicolumn{3}{c}{ M.V } & \multicolumn{5}{c}{ Bayesiano } \\
\cline { 2 - 8 } & $\hat{\boldsymbol{\theta}}$ & $\begin{array}{c}\text { Intervalo de } \\
\text { Confiança }\end{array}$ & Média & Moda & Mediana & $\begin{array}{c}\text { Desvio } \\
\text { Padrão }\end{array}$ & $\begin{array}{c}\text { Intervalo de } \\
\text { Credibilidade }\end{array}$ \\
\hline$\alpha_{0}$ & $6.3 \mathrm{e}-$ & $\begin{array}{c}(3.77 \mathrm{e}-007 ; 8.88 \mathrm{e}- \\
0.7\end{array}$ & $\begin{array}{c}5.99 \mathrm{e}- \\
007)\end{array}$ & $\begin{array}{c}5.99 \mathrm{e}- \\
0.99 \mathrm{e}-007\end{array}$ & $3.50 \mathrm{e}-014$ & $(5.99 \mathrm{e}-007 ; 6.00 \mathrm{e}-$ \\
$\alpha_{1}$ & 0.1162 & $(0.097 ; 0.1354)$ & 0.1101 & 0.1099 & 0.1011 & $5.43 \mathrm{e}-004$ & $(0.109 ; 0.111)$ \\
$\beta_{1}$ & 0.8843 & $(0.8674 ; 0.9011)$ & 0.8866 & 0.8877 & 0.8866 & $2.07 \mathrm{e}-003$ & $(0.882 ; 0.896)$ \\
\hline
\end{tabular}

As estimativas de máxima verossimilhança e bayesianas apresentaram-se próximas e $o$ intervalo de credibilidade mostrou-se mais preciso.

$\mathrm{Na}$ implementação do algoritmo Metrópolis-Hastings utilizamos o mesmo procedimento aplicado nas séries anteriores.

O critério de Gelman e Rubin foi verificado, obtendo-se $\sqrt{\hat{R}}<1.2$ para todos os parâmetros.

Nos gráficos da figura 46 apresentamos os histogramas construídos com as amostras selecionadas para os parâmetros $\alpha_{0}, \alpha_{1}$ e $\beta_{1}$, respectivamente. 

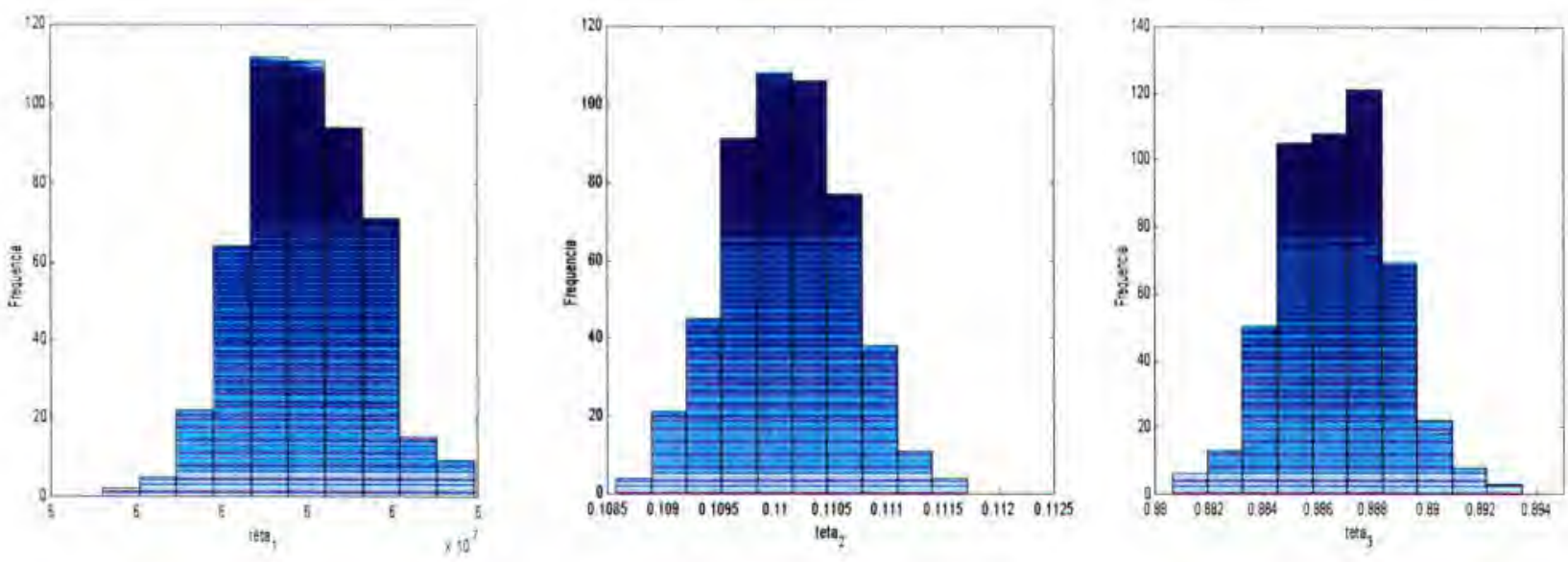

Figura 46: Distribuição a Posteriori Marginal: $\alpha_{0}, \alpha_{1}$ e $\beta_{1}$
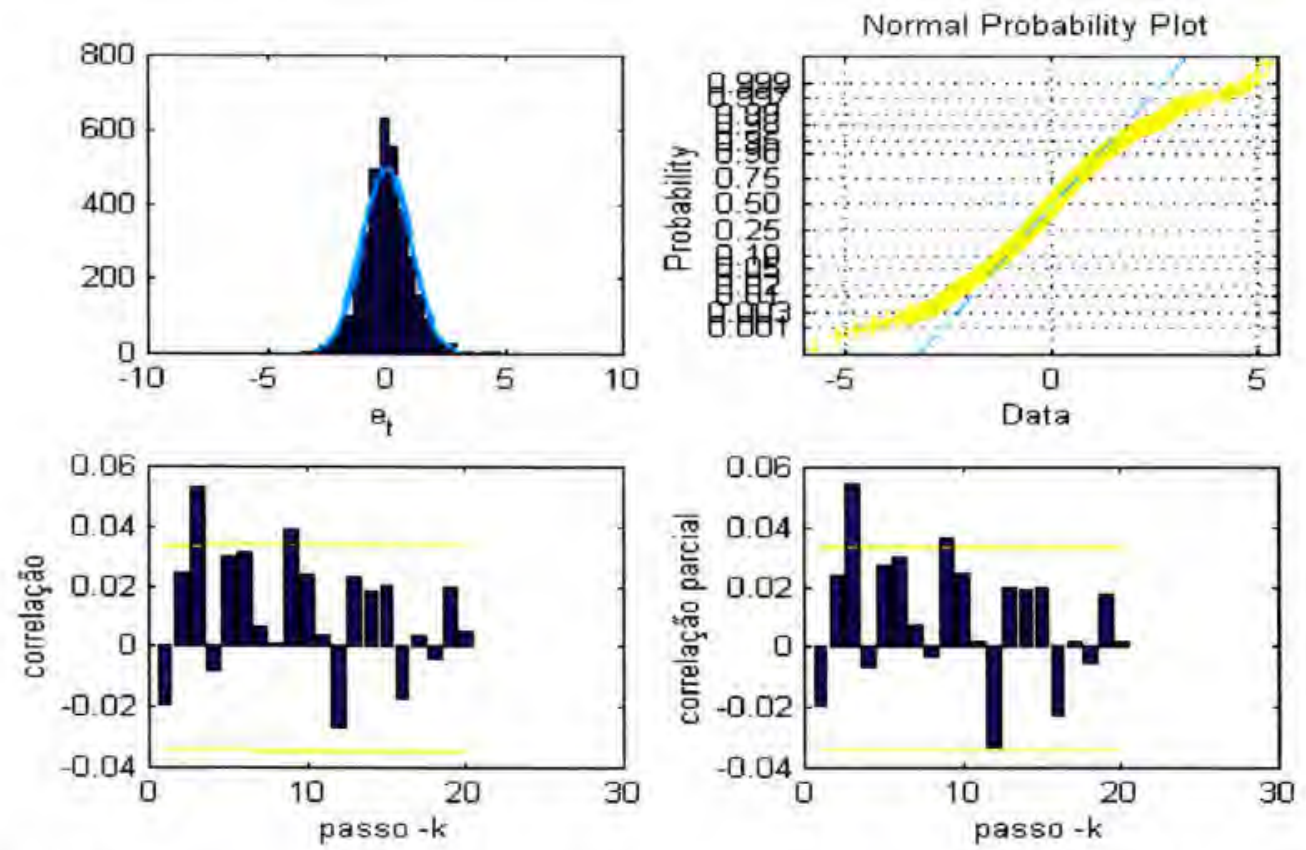

Figura 47: Histograma, gráficos normal probabilístico, auto-correlação e correlação parcial dos resíduos- série moeda

Através da figura 47 observamos que os resíduos não apresentam distribuição normal e apresentam uma pequena correlação.

A figura 48 apresenta o gráfico da volatilidade estimada para um período de 3480 dias. Os últimos 30 dias foram deixados fora do ajuste para serem utilizados no cálculo da previsão da volatilidade e do preço. 


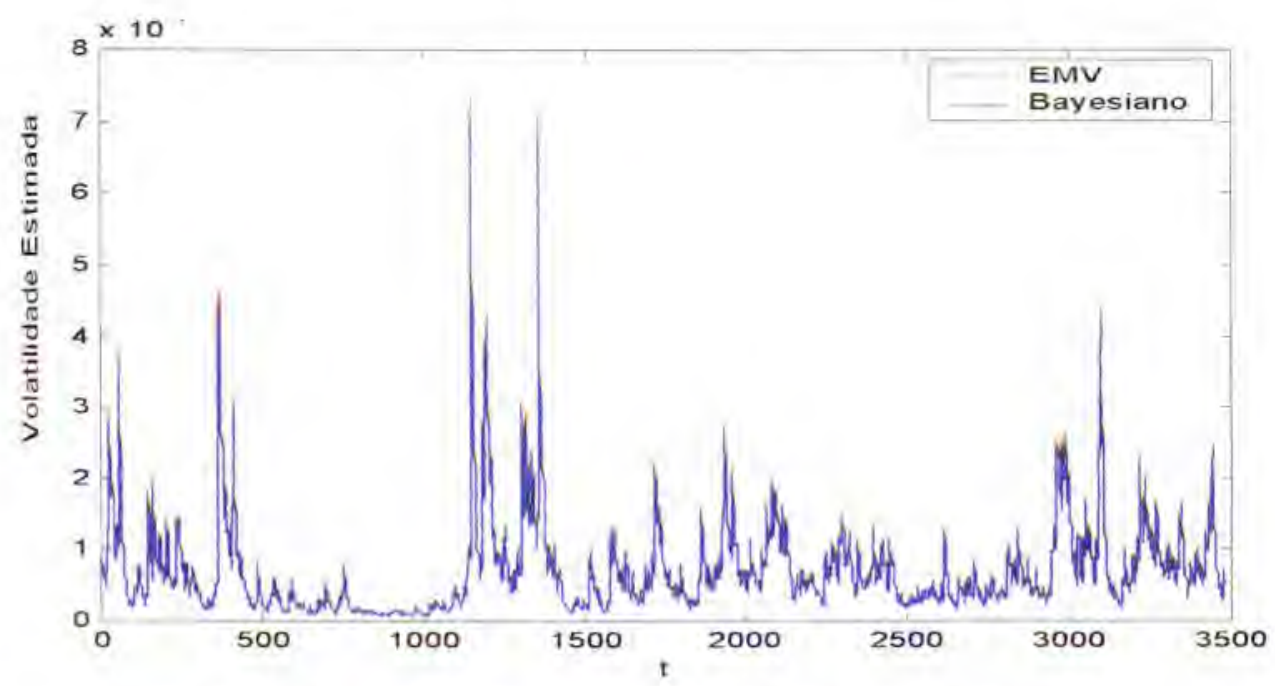

Figura 48: Gráfico da volatilidade estimada-série moeda

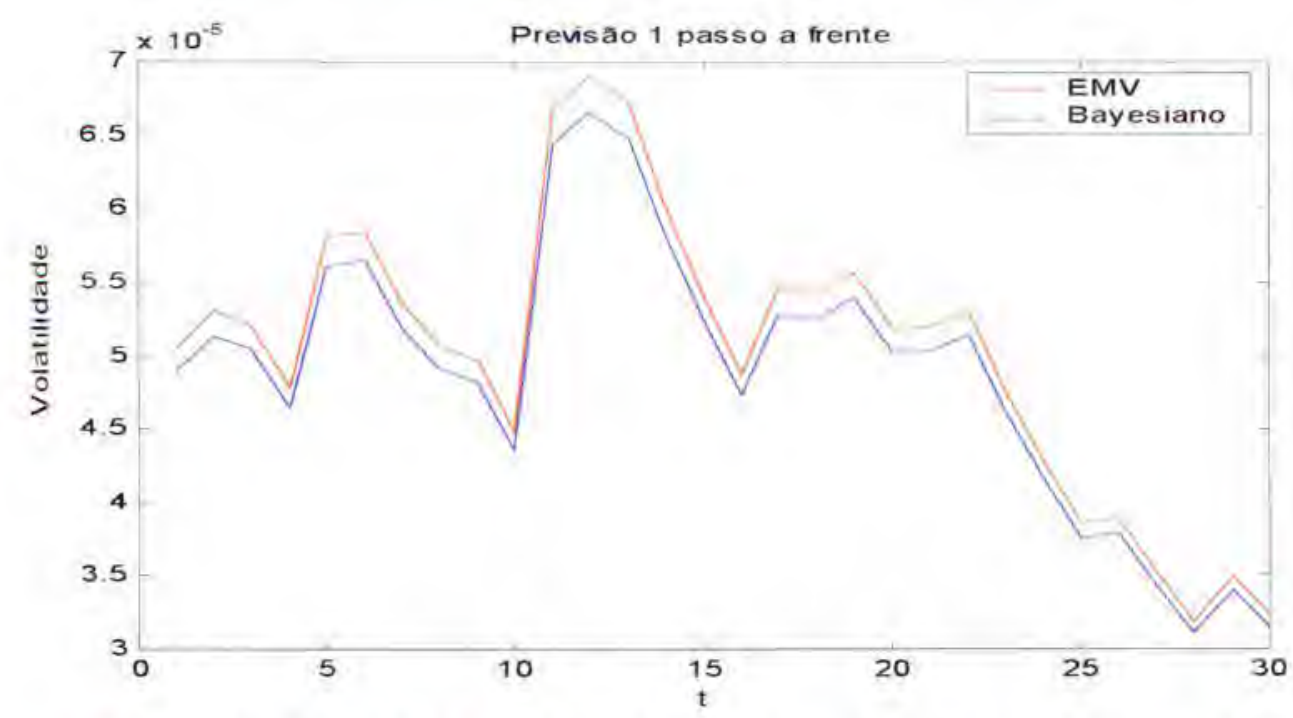

Figura 49: Previsão da volatilidade 1 passo a frente - série moeda

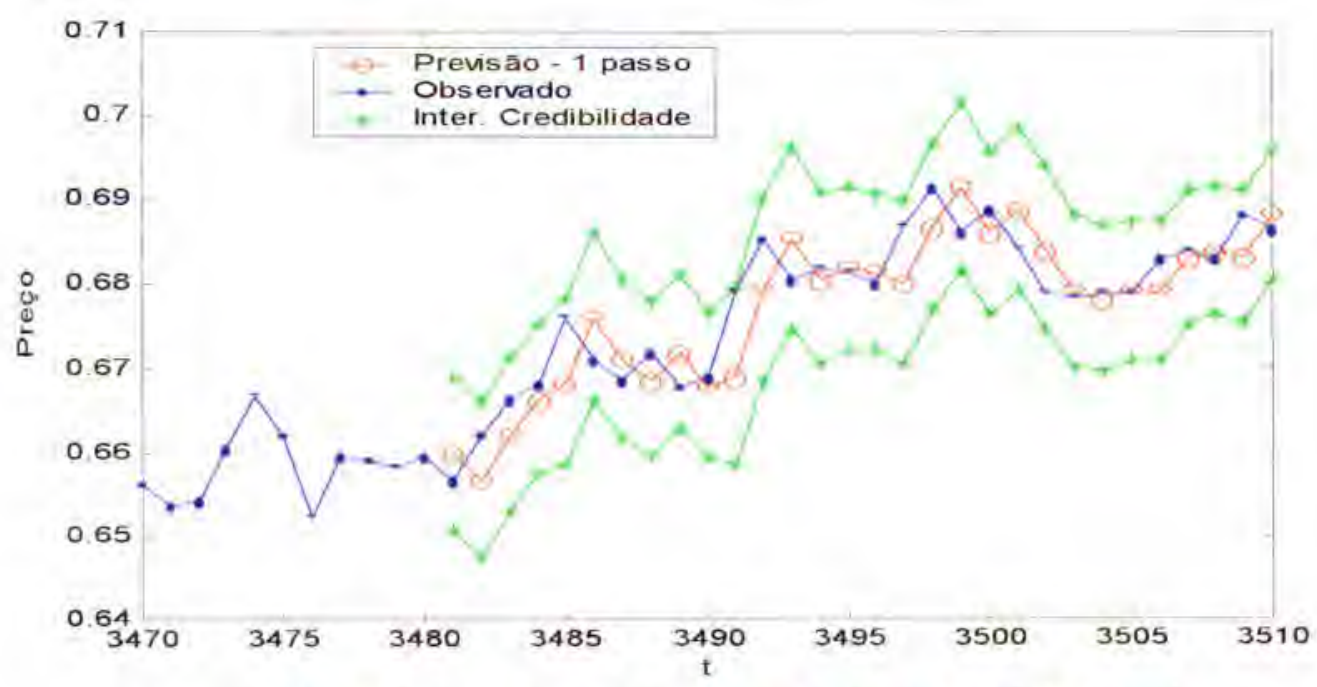

Figura 50: Previsão do preço 1 passo a frente - série moeda 
As figuras 49 e 50 apresentam a previsão um passo à frente da volatilidade e do preço, respectivamente, durante um período de 30 dias. A previsão do preço apresentada foi feita com os resultados obtidos na abordagem bayesiana.

Tabela 16: Avaliação da previsão da volatilidade e do preço - 1 passo a frente

\begin{tabular}{lccc}
\hline & Volatilidade & \multicolumn{2}{c}{ Preço } \\
\cline { 2 - 4 } & EMQ & EMQ & EMP(\%) \\
\hline Série Gerada & $5.3242 \mathrm{e}-004$ & $2.1760 \mathrm{e}-002$ & 12.277 \\
Série lbovespa & $5.1071 \mathrm{e}-005$ & $2.5986 \mathrm{e}-001$ & 3.6312 \\
Série Telebrás & $1.4401 \mathrm{e}-006$ & $1.8372 \mathrm{e}-006$ & 2.1894 \\
Série Moeda & $2.4602 \mathrm{e}-009$ & $1.7981 \mathrm{e}-005$ & 0.5133 \\
\hline
\end{tabular}

A tabela 16 apresenta os valores das funções Erro Médio Quadrático (EMQ) e Erro Médio Percentual (EMP) obtidos na análise da previsão da volatilidade e do preço. 


\section{Capítulo 6}

\section{Conclusões e Propostas Futuras}

As abordagens clássica e bayesiana para os modelos $\mathrm{ARCH}(\mathrm{p})$ são viáveis, eficientes e de fácil implementação. No caso dos modelos $\operatorname{GARCH}(p, q)$, a abordagem clássica só pode ser obtida considerando o estimador da matriz de covariância como $F^{-1}=T^{-1}\left(\sum_{t=m+1}^{T} \frac{\partial l_{t}}{\partial \boldsymbol{\theta}} \frac{\partial l_{t}}{\partial \boldsymbol{\theta}^{\prime}}\right)^{-1}$.

A abordagem bayesiana para os modelos $\operatorname{GARCH}(p, q)$ apresenta dificuldade na escolha dos parâmetros das densidades a priori devido ao grau de dependência entres esses parâmetros. No entanto, para estudos futuros com densidades mais gerais que a Normal, a abordagem bayesiana é uma ferramenta que deve ser considerada.

Como propostas futuras podemos considerar:

- Outras distribuiç̃̃es para o ruído, como $t$-student e Potência Exponencial;

- Análise de modelos de regressão com erros ARCH e GARCH;

- Aplicação das técnicas utilizadas para modelos com assimetria na variância (EGARCH);

- Comparação dos modelos ARCH e GARCH com outros modelos como, por exemplo, modelos com volatilidade estocástica. 


\section{Bibliografia}

Akaike, H. (1974). A new look at the statistical identification model, IEEE Trans. on Automatic Control, Vol. 19, pp. 716-723.

Bollerslev, T. (1986). Generalized Autoregressive Conditional Heteroskedasticity, Journal of Econometrics, Vol. 31, pp. 307-327.

Bollerslev, T; Chou, R.Y.; Kroner, K.F. (1992). ARCH Modeling in Finance: a review of the theory and empirical evidence, Journal of Econometrics, Vol. 52, pp. 5-59.

Chib, S.; Greenberg, E. (1995). Understanding the Metropolis-Hastings Algorithm, The American Statistician, Vol. 49, No. 4, pp. 327-335.

Engle, R.F. (1982). Autoregressive Conditional Heteroscedasticity with Estimates of the Variance of UK Inflation, Econometrica, Vol. 50, pp. 987-1007.

Gelman, A.; Rubin, D.B. (1992). Inference from Iterative Simulation Using Multiple Sequences, Statistical Science, Vol. 7, No. 4, pp. 457-511

Geweke, J. (1986a). Exact inference in the inequality constrained normal linear regression model, Journal of Applied Econometrics, Vol. 1, pp. 127-141.

Geweke, J. (1986c). Bayesian inference in econometric models using Monte Carlo integration, Manuscript (Duke University, Durham, NC).

Geweke, J. (1989). Exact Predictive densities for linear models with ARCH disturbances, Journal of Econometrics, Vol. 40, pp. 63-86

Hamilton, J. D.; Susmel, R. (1994). Autoregressive conditional heteroskedasticity and changes in regime, Journal of Econometrics, Vol. 64, pp. 307-333.

Harvey, A C. (1992). Time Series Models, 2th ed., Harvester Wheatsheaf, London.

Luenberger, D. G. (1984). Linear and Nonlinear Programming. Addison-Wesley, Reading, 
Mass.

Lee, J. H. H. (1992). A survey of Arch and Related Models. Department of Economics \& Finance. Faculty of Business. Melbourne-Australia.

Migon, H. S.; Mazucheli, J. (1999). Modelos GARCH Bayesianos: Métodos Aproximados e Aplicações, Revista de Econometria, Vol. 19, No. 1, pp. 111-138.

Nakatsuma, T. (2000). Bayesian analysis of ARMA-GARCH models: A Markov chain sampling approach. Journal of Econometrics, Vol. 95, pp. 57-69.

Schwarz, G. (1978). Estimating the dimension of a model. The Annals of Statistics, Vol. 6, No. 2, pp. 461-464. 